\title{
The Detection and Direction Finding of Multiple Weak Sinusoidal Signals with a Circular Antenna Array
}

\author{
by \\ Sean Stamplecoskie, B. Eng. \\ A thesis submitted to the \\ Faculty of Graduate Studies and Research \\ in partial fulfillment of the requirements for the degree of \\ Master of Applied Science
}

Ottawa-Carleton Institute for Electrical and Computer Engineering

Department of Electronics

Faculty of Engineering

Carleton University

Ottawa, Canada

June 2008

(C) Sean Stamplecoskie, 2008 


$\begin{array}{ll}\begin{array}{l}\text { Library and } \\ \text { Archives Canada }\end{array} & \begin{array}{l}\text { Bibliothèque et } \\ \text { Archives Canada }\end{array} \\ \begin{array}{l}\text { Published Heritage } \\ \text { Branch }\end{array} & \begin{array}{l}\text { Direction du } \\ \text { Patrimoine de l'édition }\end{array} \\ \begin{array}{l}\text { 395 Wellington Street } \\ \text { Ottawa ON K1A 0N4 } \\ \text { Canada }\end{array} & \begin{array}{l}\text { 395, rue Wellington } \\ \text { Ottawa ON K1A 0N4 } \\ \text { Canada }\end{array}\end{array}$

Your file Votre référence ISBN: 978-0-494-44058-2

Ourfile Notre référence

ISBN: 978-0-494-44058-2

NOTICE:

The author has granted a nonexclusive license allowing Library and Archives Canada to reproduce, publish, archive, preserve, conserve, communicate to the public by telecommunication or on the Internet, loan, distribute and sell theses worldwide, for commercial or noncommercial purposes, in microform, paper, electronic and/or any other formats.

The author retains copyright ownership and moral rights in this thesis. Neither the thesis nor substantial extracts from it may be printed or otherwise reproduced without the author's permission.
AVIS:

L'auteur a accordé une licence non exclusive permettant à la Bibliothèque et Archives Canada de reproduire, publier, archiver, sauvegarder, conserver, transmettre au public par télécommunication ou par l'Internet, prêter, distribuer et vendre des thèses partout dans le monde, à des fins commerciales ou autres, sur support microforme, papier, électronique et/ou autres formats.

L'auteur conserve la propriété du droit d'auteur et des droits moraux qui protège cette thèse. $\mathrm{Ni}$ la thèse ni des extraits substantiels de celle-ci ne doivent être imprimés ou autrement reproduits sans son autorisation.
In compliance with the Canadian Privacy Act some supporting forms may have been removed from this thesis.

While these forms may be included in the document page count, their removal does not represent any loss of content from the thesis.
Conformément à la loi canadienne sur la protection de la vie privée, quelques formulaires secondaires ont été enlevés de cette thèse.

Bien que ces formulaires aient inclus dans la pagination, il n'y aura aucun contenu manquant.

\section{Canada}




\section{Abstract}

Signal detection and bearing estimation are rich research areas with applications in sonar, military surveillance, navigation and search and rescue.

For the case of a single sinusoid in additive white Gaussian noise, with all parameters unknown, the periodogram is the maximum likelihood estimator. Additionally, the periodogram is a non-parametric technique, providing good parameter estimation under low signal to noise conditions. When multiple sinusoids are present, high resolution techniques such as Auto-Regressive Moving Average (ARMA) and MUltiple SIgnal Classification (MUSIC) provide accurate parameter estimates. However, when the sinusoids are weak, these parametric models produce large estimation errors.

When the minimum frequency separation of the sinusoids exceeds four FFT bins and appropriate filtering is employed, the single sinusoid parameter estimation bounds of the periodogram can be applied to the multiple sinusoid case.

In this thesis, a periodogram based architecture is developed, providing sinusoidal detection and bearing estimation for multiple weak sinusoids with a circular antenna array. Theoretical performance bounds are derived and compared against simulation results. 


\section{Acknowledgements}

First and foremost, I would like to thank my supervisor Prof. Jim Wight for giving me the opportunity to study under his direction. I would also like to thank Mr. Kenneth Moreland of Rockwell Collins Government Systems Canada Inc. for his mentoring and insight into the many aspects of digital signal processing. I would like to thank Mr. Andrew Mudry of DRDC Ottawa for his guidance, support and encouragement throughout this process. Additionally, I would like to thank Mr. Alain Joyal of DRDC Ottawa for his assistance with Matlab programming and insight into the practical implementation of this work. Last but not least, I would like to thank my wife Jennifer for her understanding and assistance throughout the course of this work. 


\section{Contents}

1 Introduction 1

1.1 Background ........................... 1

1.2 Thesis Objectives ..................... 2

1.3 Thesis Contributions ...................... 3

1.4 Thesis Outline...................... 6

2 Background $\quad 7$

2.1 Signal Model ....................... 7

2.1.1 The Received Signal . . . . . . . . . . . . . 7

2.1.2 The Baseband Signal . . . . . . . . . . . . . 9

2.1.3 The Digital Baseband Signal . . . . . . . . . . . . . 14

2.2 Sinusoidal Parameter Estimation . . . . . . . . . . . . 16

2.2.1 Performance Bounds For Sinusoidal Parameter Estimation . . 17

2.2.2 Performance Bounds for Single Sinusoid Estimation . . . . . . 18

2.2.3 Maximum Likelihood Estimation for a Single Sinusoid . . . . . 20

2.2.4 Performance Bounds for Multiple Sinusoids . . . . . . . . . . 23

2.3 Sinusoid Detection Theory . . . . . . . . . . . . 26

2.3.1 Primary Signal Detection Approaches . . . . . . . . . . 28 
2.3.2 The GLRT Detector for Single Complex Sinusoid . . . . . 29

The GLRT for Unknown Amplitude and Phase . . . . . . 30

The GLRT for Unknown Frequency, Amplitude and Phase . . 33

Single Tone Detection for Averaged Periodograms . . . . . . . 34

2.3.3 Detection of Multiple Sinusoids . . . . . . . . . . 35

2.4 Bearing Estimation Performance Bounds . . . . . . . . . . 37

2.4.1 The CRLB for Bearing Estimation . . . . . . . . . . 39

2.4.2 CRLB for Bearing of a Single Sinusoid . . . . . . . . . . 40

Multiple Sinusoid Bearing Estimation Performance . . . . . 44

3 Detection and Bearing Estimation Algorithms 45

3.1 PSD Computation $\ldots \ldots \ldots \ldots \ldots \ldots \ldots$

3.1.1 Signal Segmentation and Windowing . . . . . . . . 47

3.1.2 Fast Fourier Transform (FFT) Computation . . . . . . . . 47

3.1 .3 Cross-Correlation . . . . . . . . . . . . . . . . . 49

3.1 .4 Power Spectral Density (PSD) Averaging . . . . . . . . . . 51

The Bartlett Method . . . . . . . . . . . . . . . . . 51

The Welch Method . . . . . . . . . . . . . . . 52

3.2 Sinusoid Detection $\ldots \ldots \ldots \ldots \ldots \ldots \ldots$

3.2.1 The Single Channel Periodogram Detector . . . . . . . . 52

3.2.2 The Multichannel Averaged Periodogram Detector _. . . . 53

3.3 Bearing Estimation . . . . . . . . . . . . . . . . . 54

3.3.1 The Interferometer Technique . . . . . . . . . . . . 55

3.3.2 The Eigen-Decomposition Technique . . . . . . . . . 58

Computation of the Eigenvalues . . . . . . . . . . 60

Computation of the Eigenvectors . . . . . . . . . . 61 
Computation of the Bearing Estimates . . . . . . . . . 61

3.3.3 Eigen-Decomposition Technique using the Power Method . . . 64

4 Results $\quad 66$

4.1 Detection Results . . . . . . . . . . . . . . . . . 67

4.1.1 Single Sinusoid with Frequency at Center Bin . . . . . . . 68

Single Channel Detection . . . . . . . . . . . . . . . . 68

Multiple Channel Detection . . . . . . . . . . . . . . 72

4.1.2 Single Sinusoid with Random Frequency . . . . . . . . . 77

Single Channel Detection . . . . . . . . . . . . . . 77

Multiple Channel Detection . . . . . . . . . . . . . 80

4.1.3 Single Sinusoid with Random Frequency and Random Amplitude 83

Single Channel Detection . . . . . . . . . . . . . . . . 83

Multiple Channel Detection . . . . . . . . . . . . . 85

4.1.4 Multiple Sinusoids with Random Frequency $\ldots \ldots \ldots$. . . 86

Single Channel Detection . . . . . . . . . . . . . . . 87

Multiple Channel Detection . . . . . . . . . . . . 88

4.1 .5 Conclusions . . . . . . . . . . . . . . . . . . . 89

4.2 Bearing Estimation Results $\ldots \ldots \ldots \ldots \ldots$

4.2.1 Single Sinusoid with Frequency at Center Bin . . . . . . . . 91

Sinusoid with Bearing $0 \ldots \ldots \ldots$. . . . . . . 91

Sinusoid with Bearing $\pi / 8 \ldots \ldots \ldots \ldots \ldots$

Sinusoid with Bearing $\pi / 4 \ldots \ldots \ldots \ldots$. . . . . . 94

4.2.2 Single Sinusoid with Random Frequency . . . . . . . . 95

Sinusoid with Bearing $0 \ldots \ldots \ldots \ldots$

Sinusoid with Bearing $\pi / 8 \ldots \ldots \ldots \ldots$ 
Sinusoid with Bearing $\pi / 4 \ldots \ldots \ldots \ldots$. . . . . 97

4.2.3 Multiple Sinusoids with Random Frequency . . . . . . . . 98

Sinusoid with Bearing $0 \ldots \ldots \ldots \ldots$

Sinusoid with Bearing $\pi / 8 \ldots \ldots \ldots \ldots$

Sinusoid with Bearing $\pi / 4 \ldots \ldots \ldots$. . . . . . . . 101

4.2.4 Effect of Sinusoid Separation on Bearing Estimation Error . . 102

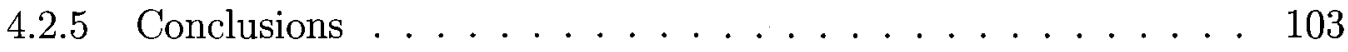

$\begin{array}{lll}5 & \text { Conclusions and Future Work } & 105\end{array}$

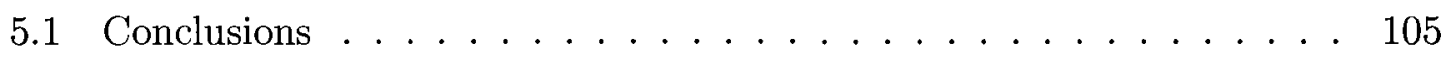

5.2 Future Work . . . . . . . . . . . . . . . . . . . . 107

5.2.1 Algorithm Implementation . . . . . . . . . . . . . . 107

5.2 .2 Minimum Sinusoid Separation . . . . . . . . . . . . 107

5.2.3 Channelization to Increase Bearing Estimation Accuracy . . 108

5.2 .4 Fast Orthogonal Search . . . . . . . . . . . . . . . . 109

5.2.5 Three Antenna Element Array . . . . . . . . . . . . . . 109

5.2.6 The Conjugate Gradient Method . . . . . . . . . . . . 110

A Probability and Random Process Theory $\quad 116$

A.1 Deterministic vs. Stochastic Processes . . . . . . . . . . 116

A.1.1 Deterministic Process . . . . . . . . . . . . . . . 116

A.1.2 Stochastic Process . . . . . . . . . . . . . 117

A.2 Stochastic Process Properties . . . . . . . . . . . 117

A.2.1 The Central Limit Theorem . . . . . . . . . . . . . . 119

A.2.2 The Gaussian Distribution . . . . . . . . . . . 120

A.3 The Cramer-Rao Lower Bound and Estimator Performance . . . . . . 121 
A.3.1 Characteristics of Estimator Performance . . . . . . . . . . 122

A.3.2 Scalar CRLB . . . . . . . . . . . . . . . 122

A.3.3 Vector CRLB . . . . . . . . . . . . . . . 123

B Spectral Estimation Theory 128

B.1 Spectrum . . . . . . . . . . . . . . . . 128

B.2 The Fourier Transform . . . . . . . . . . . . . . . . . . . . . 129

B.3 The Discrete Fourier Transform . . . . . . . . . . . . . . . . . . . 129

B.3.1 The Fast Fourier Transform . . . . . . . . . . . . . . . . 130

B.3.2 Windowing and the Discrete Fourier Transform . . . . . . 130

B.3.3 Sampling and the Discrete Fourier Transform . . . . . . . . 133

B.3.4 Equivalent Noise Bandwidth . . . . . . . . . . . . 134

B.4 Alternative Windows for Spectral Estimation . . . . . . . . . . . . . . 134

B.5 Zero Padding . . . . . . . . . . . . . . . . 137

B.6 Parseval's Theorem . . . . . . . . . . . . . . . 137

B.7 Power Spectral Density . . . . . . . . . . . . . . . . . . 138

B.8 The Periodogram . . . . . . . . . . . . . . . . 138

B.8.1 Modified Periodogram . . . . . . . . . . . 139

B.8.2 Averaged Periodograms . . . . . . . . . . . 139

The Bartlett Method . . . . . . . . . . . . . . 140

The Welch Method . . . . . . . . . . . . . 141

C Characteristic polynomial for a 4x4 Matrix $\quad 146$

D Solving Quartic Equations $\quad 151$

D.1 Convert the quartic equation to a depressed quartic equation . . . . 152

D.2 Factor the quartic equations into two quadratic equations . . . . . 153 
D.3 Create an expression for the quadratic coefficients . . . . . . . . 154

D.4 Solving the cubic equation $\ldots \ldots \ldots \ldots \ldots \ldots \ldots$

D.4.1 Solving a cubic equation with three real roots . . . . . 156

D.4.2 Solving a cubic equation with one real root and two complex roots . . . . . . . . . . . . . . . 158

D.5 Solving the quartic equation $\ldots \ldots \ldots \ldots \ldots \ldots$ 


\section{List of Figures}

2.1 The circular antenna array and one signal arriving from a bearing $\phi$. . 8

2.2 Each receiver tunes the signals to baseband with a narrowband quadrature demodulator .......................... 10

2.3 The spectrum of the predetection signal . . . . . . . . . 10

2.4 The spectrum of the received signal after tuning to baseband . . . . 13

2.5 The discrete-time spectrum of the in-phase baseband signal. . . . . . 16

2.6 Example of a ROC diagram. . . . . . . . . . . . . . 27

2.7 Detection performance comparison. . . . . . . . . . . . 37

2.8 Example of a curve $\mathbf{A}(\phi)$, an arc on the curve $\mathbf{s}(\phi)$ and its unit tangent $\mathbf{u}(\phi) \ldots \ldots \ldots \ldots \ldots \ldots \ldots$

3.1 The signal processing model, where the subscripts $x, y$ represent the antenna numbers for an antenna pair. . . . . . . . . . . 46

3.2 The processing elements of the PSD Computation Block. . . . . . . 46

3.3 The solid line represents the DTFT spectrum, while the stem plot indicates the discrete Discrete Fourier Transform (DFT) values. The three plots indicate the spectrum of the (a) input signal, (b) window function and (c) windowed input sequence. . . . . . . . . . . . 48

3.4 Multichannel Power Spectral Density matrix for v[n]. . . . . . . 50 
3.5 Ambiguity of $\tan ^{-1}$ due to the combined sign term. . . . . . . 57

3.6 Multichannel PSD matrix utilization for four channel Interferometer. $\quad 58$

3.7 Cost function and gradient for a signal with bearing pi/4. . . . . . 64

4.1 Receiver Operating Characteristic (ROC) for single channel Bartlett detection with frequency at center of FFT bin. . . . . . . . . 69

4.2 ROC for single channel Welch detection with frequency at center of FFT bin. . . . . . . . . . . . . . . . . . . . .

4.3 Comparison of ROCs for single channel Bartlett and Welch detection with frequency at center of FFT bin. . . . . . . . . . . .

4.4 ROC for multiple channel Bartlett detection with frequency at center of FFT bin. . . . . . . . . . . . . . . . . . 73

4.5 ROC for multiple channel Welch detection with frequency at center of FFT bin. . . . . . . . . . . . . . . . . .

4.6 Comparison of ROCs for multiple channel Bartlett and Welch detection with frequency at center of FFT bin. . . . . . . . . . 75

4.7 ROC for single channel Welch detection with frequency at center of FFT bin. . . . . . . . . . . . . . . . . 76

4.8 ROC for single channel Bartlett detection with random frequency. . . 77

4.9 ROC for single channel Welch detection with random frequency. . . . 78

4.10 ROC comparison for single channel detection with random frequency. $\quad 79$

4.11 ROC comparison for multiple channel detection with random frequency. 81

4.12 ROC comparison single vs. multiple channel detection with random frequency . . . . . . . . . . . . . . . . . . 82

4.13 ROC for single channel detection with random frequency and random amplitude. . . . . . . . . . . . . . . . . 
4.14 ROC for multiple channel detection with random frequency and ran-

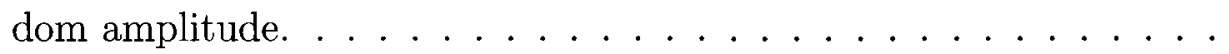

4.15 Comparison of ROCs for random frequency and random amplitude

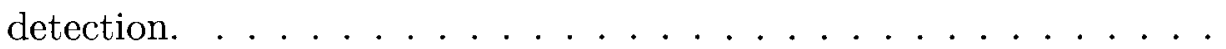

4.16 ROC comparison for single channel detection with multiple sinusoids of random frequency and random amplitude. . . . . . . . . .

4.17 ROC comparison for multiple channel detection with multiple sinusoids of random frequency and random amplitude. . . . . . . . . . . . 88

4.18 Linear representation of bearing estimation performance for a single sinusoid at the center of an FFT bin, from bearing $0 . \ldots \ldots$

4.19 Logarithmic representation of bearing estimation performance for a single sinusoid at the center of an FFT bin, from bearing $0 \ldots$. . . . 92

4.20 Bearing estimation performance for a single sinusoid at the center of an FFT bin, from bearing $\pi / 8 . \ldots \ldots \ldots \ldots$

4.21 Bearing estimation performance for a single sinusoid at the center of an FFT bin, from bearing $\pi / 4 \ldots \ldots \ldots \ldots$

4.22 Bearing estimation performance for a single sinusoid with random frequency, from bearing $0 \ldots \ldots \ldots \ldots$

4.23 Bearing estimation performance for a single sinusoid with random frequency, from bearing $\pi / 8 \ldots \ldots \ldots \ldots$

4.24 Bearing estimation performance for a single sinusoid with random frequency, from bearing $\pi / 4 \ldots \ldots \ldots \ldots$

4.25 Bearing estimation performance for multiple sinusoids with random frequency, from bearing $0 \ldots \ldots \ldots \ldots$ 
4.26 Bearing estimation performance for multiple sinusoids with random frequency, from bearing $\pi / 8 \ldots \ldots \ldots$. . . . . . . . 100

4.27 Bearing estimation performance for multiple sinusoids with random frequency, from bearing $\pi / 4 \ldots \ldots \ldots$. . . . . . . . . . 101

4.28 Effect of minimum sinusoid separation on bearing estimation error. . 103

A.1 The normalized Gaussian density function. . . . . . . . . . . . 120

B.1 The rectangular window function for $\mathrm{N}=64$, a) Time-Domain b) Frequency Response c) Shifted Frequency Response . . . . . . . . . . 132

B.2 Time and Frequency Response of a Rectangular Windowed Sinusoid, for $f_{0}=0.1$ and $\mathrm{N}=64 \ldots \ldots \ldots \ldots$

B.3 Scalloping loss for the Rectangular Window, where $N=64 \ldots \ldots$. . . 134

B.4 Time and frequency response of the Rectangular, Blackman, Hann and Hamming window functions, for $\mathrm{N}=64 \ldots \ldots \ldots$

B.5 The Bartlett periodogram averaging method for $\mathrm{N}=32, \mathrm{~L}=8$ and $\mathrm{M}$ $=4$.

B.6 a) The periodogram for $\mathrm{N}=4096$. b) The Bartlett averaged periodogram for $\mathrm{M}=8$ and $\mathrm{L}=512$. c) Bartlett averaged periodogram for $\mathrm{M}=32$ and $\mathrm{L}=128 \ldots \ldots \ldots \ldots$. . . . . . . . . 142

B.7 The Welch periodogram averaging method for $\mathrm{N}=32, \mathrm{~L}=8, \mathrm{M}=7$ and overlap $=50 \% \ldots \ldots \ldots \ldots \ldots$

B.8 a) The periodogram for $\mathrm{N}=4096$. b) The Welch averaged periodogram for $\mathrm{M}=15$ and $\mathrm{L}=512$. c) The Welch averaged periodogram for $\mathrm{M}$ $=63$ and $\mathrm{L}=128 \ldots \ldots \ldots \ldots \ldots$ 


\section{List of Tables}

B.1 Window functions of the Rectangular, Blackmanm Hann and Hamming windows. . . . . . . . . . . . . . . . 135

B.2 Key window parameters for the Rectangular Blackman, Hann and Hamming window Functions. . . . . . . . . . . . . . . 135 


\section{List of Acronyms}

ADC Analog to Digital Converter.

AOA Angle of Arrival.

ARMA Auto-Regressive Moving Average.

AWGN Additive White Gaussian Noise.

CRLB Cramer Rao Lower Bound.

CWGN Complex White Gaussian Noise.

dB decibel.

DDC Digital Downconverter.

DFT Discrete Fourier Transform.

ESPRIT Estimation of Signal Parameter via Rotational Invariance Techniques

FFT Fast Fourier Transform.

FOS Fast Orthogonal Search.

FPGA Field Programmable Gate Array. 
GLRT Generalized Likelihood Ratio Test.

Hann Hanning Window.

IF Intermediate Frequency.

i.i.d. identically independently distributed.

LO Local Oscillator.

ML Maximum Likelihood.

MLE Maximum Likelihood Estimate.

MUSIC MUltiple SIgnal Classification.

NP Neyman-Pearson.

PDF Probability Density Function.

PSD Power Spectral Density.

RMS Root Mean Square.

RMSE Root Mean Square Error.

ROC Receiver Operating Characteristic.

SETI Search for Extraterrestrial Intelligence.

SNR Signal to Noise Ratio.

SPV Source Position Vector. 


\section{Chapter 1}

\section{Introduction}

\subsection{Background}

Signal detection and bearing estimation are rich research areas with applications in sonar, military surveillance, navigation and search and rescue and the Search for Extraterrestrial Intelligence (SETI).

Signal detection theory is the application of statistical hypothesis testing to the detection of signals in noise[1]. Signal detection is a key component of many signal processing applications. The results of the signal detection process are typically used as input for decision making and information extraction (such as bearing estimation). Signal detection can be performed with a single channel or over multiple channels in array processing configurations. The use of single channel and multiple channel detection is investigated in this thesis.

Bearing estimation is the determination of the bearing of an emitting source through the suitable processing of the received signal. Typically, a bearing estimator utilizes an antenna array, the output of which contains multiple scaled versions of the emitter signal. The multiple versions of the emitter signal result from geometric differences between the emitter and the individual antennas of the array. The differences between the observed signals on the individual antenna elements are used to 
estimate the Angle of Arrival (AOA) of the emitter. The AOA information can then be processed by a position location system for triangulation of the emitter.

Various detection strategies and bearing estimation techniques can be applied to a specific emitter source, with varying degrees of performance. Variables affecting the performance of detection strategies and direction finding techniques include the number of signals present, their Signal to Noise Ratios (SNRs), the signal spatial separation, the signal durations, the signal bandwidths and the antenna array. Additionally, practical limitations due to signal processing hardware and memory requirements must be considered.

\subsection{Thesis Objectives}

The detection and bearing estimation of multiple weak tones has several practical applications. Examples include beacons for short range tracking of military assets and the location of remote sensors and telemetry equipment.

The selection of a particular signal detection and bearing estimation technique is largely dependant upon the known and unknown parameters of the signal of interest. For example, when designing a sinusoid detector, design choices are impacted by the availability of signal parameters such as amplitude, frequency, phase, bearing and relative frequency separation. The signals investigated in this thesis are sinusoidal, with unknown amplitude, frequency, phase and bearing. A maximum of 10 signals are present within a frequency range $55-60 \mathrm{MHz}$ and the signals have a minimum separation of $12.5 \mathrm{kHz}$. The signals are assumed stable for the duration of the observation interval. The signals are corrupted by Additive White Gaussian Noise (AWGN) and the signal to noise ratio is $-45 \mathrm{~dB}$ or greater, relative to the $5 \mathrm{MHz}$ bandwidth.

Typically, an antenna array is used to estimate the AOA of a detected signal. 
In mobile applications, the size, number, and spatial pattern of antenna elements is limited by the vehicular platform. These restrictions are most stringent in aerial platforms, where performance is restricted by size, weight and power. The antenna array studied in this report is a four element circular antenna array with a radius of one meter.

The aim of this thesis is to investigate the performance of various signal detection and direction finding techniques for multiple, relatively spaced sinusoids, with a fourchannel circular array.

\subsection{Thesis Contributions}

Detection and bearing estimation are mature fields of research, of which several aspects related to this thesis have been studied and well documented. For example, extensive research has been performed in the field of sinusoidal parameter estimation [2], [3]. However, the majority of the literature focuses on single sinusoid applications, under large SNR conditions. Similarly, weak sinusoids have been studied extensively for submarine tracking applications [4], [5]. However, the antenna array used in submarinal applications is the towed linear array, which is substantially different from the circular array studied in this thesis. Another application, Local Oscillator (LO) detection, is well developed for use in bug sweeping and similar surveillance applications. A common implementation is to sweep across the frequencies of interest using a very narrow filter and to then perform detection using an energy detector [6]. While this technique is effective, it is typically implemented with analog components, and therefore does not provide a rapid response and has limited flexibility.

The work presented in this thesis provides a comprehensive analysis of detection and bearing estimation for multiple weak sinusoids using a circular antenna array. 
Several spectral analysis and signal processing concepts are consolidated and built upon to develop an optimal detection and bearing estimation strategy for weak signal conditions. Additionally, the strategy studied in this thesis combines the detection and bearing estimation processes, performing both operations on the same block of input data, improving efficiency over traditional techniques.

In deriving the optimal sinusoid parameter estimator, a first principles approach is employed. The Cramer Rao Lower Bound (CRLB) is used to derive the Maximum Likelihood Estimate (MLE) for a single sinusoid with unknown amplitude, frequency and phase in AWGN. The MLE is shown to be the periodogram and performance bounds for the Maximum Likelihood (ML) detection are presented. The sinusoid detection theory is then expanded to cover the multiple sinusoid case, where it is shown that using appropriate filtering and maintaining a minimum frequency separation of four FFT bins, the single sinusoid performance bounds can be applied. The CRLB is also used to provide an asymptotic lower bound on the bearing estimation performance of a circular antenna array.

The implementation studied in this thesis is a four channel circular array. The input data is recorded for a one second duration at a rate of $5 \mathrm{MHz}$ complex and the channels are assumed to be phase coherent. A 32768 point FFT is implemented to provide sufficient signal processing gain for sinusoid detection and bearing estimation. Using this configuration, the minimum signal separation is 81 FFT bins. This frequency separation greatly exceeded the required minimum separation of four FFT bins.

Sinusoid detection is performed in conjunction with two spectral averaging techniques, the Bartlett method and the Welch method (uses a Hanning Window (Hann) window). Monte Carlo simulations are performed for the case of a single sinusoid 
at the center of a FFT bin, a single sinusoid with random frequency, a single sinusoid with random frequency and amplitude; and multiple sinusoids with random frequency and amplitude. Where applicable, the results are compared with theoretical performance curves and shown to be in very good agreement. By combining the four channels, a non-coherent processing gain of 3 decibel (dB) (5 log [number of antennas]) is realized. Additionally, for sinusoids located at the center of a FFT bin the Bartlett method demonstrates superior performance. However, when the frequency is randomized, the Welch method provides significantly better performance than the Bartlett method.

Two bearing estimation techniques are investigated in this thesis, the four channel interferometer and the eigen-decomposition techniques. Bearing estimation simulations were performed for a single sinusoid at the center of a FFT bin, a single sinusoid with random frequency and multiple sinusoids with random frequency. Additionally, bearing estimation is studied for signals arriving from bearings $0, \frac{\pi}{8}$ and $\frac{\pi}{4}$. Both bearing estimation techniques provide similar performance under high SNR conditions, with the eigen-decomposition technique performing slightly better. Bearing error is presented as a function of sinusoid bearing. Both techniques exhibit their highest error when the sinusoid bearing is 0 , which corresponds to the "end-fire" condition. The best case condition is $\frac{\pi}{4}$. At low SNRs, a threshold effect is observed, typically below an input SNR of $-48 \mathrm{~dB}$. The eigen-decomposition technique exhibits the threshold effect at levels less than or equal to the levels observed for the interferometer. This is an important result, as a much greater performance improvement can be achieved by the eigen-decomposition technique when the signals of interest are very weak. Similar to the detection results, the Bartlett method exhibits greater performance for sinusoids centered on an FFT bin and the Welch method provides greater performance 
for the general case of sinusoids with random frequency. The CRLB is an asymptotic bound and is shown to be a coarse bound on bearing estimation for the interferometer and eigen-decomposition techniques.

\subsection{Thesis Outline}

This thesis is divided into 5 chapters and is organized as follows:

Chapter 1 contains the thesis introduction.

Chapter 2 contains background information including a description of the signal processing model, sinusoid parameter estimation theory, sinusoid detection theory and bearing estimation theory.

Chapter 3 provides a description of the sinusoid detection and bearing estimation algorithms evaluated in this thesis.

Chapter 4 presents the sinusoid detection and bearing estimation simulation results, for various signal conditions and signal processing algorithms. Where appropriate, the simulated results are compared against their theoretical performance estimates/bounds.

Chapter 5 provides a summary of the work presented and suggestions for future work. 


\section{Chapter 2}

\section{Background}

\subsection{Signal Model}

The signal model describes the received signal at the array input as well as the frontend processing required to produce baseband data for signal detection and direction finding.

\subsubsection{The Received Signal}

The circular antenna array consists of $\mathbf{X}$ elements, each located a distance $\mathbf{R}$ from the center of the array. The angular separation of the antenna elements is $\frac{2 \pi}{\mathbf{X}}$ radians. A four channel circular antenna array (Adcock array) is shown in Figure 2.1.

The signal $\mathrm{s}(\mathrm{t})$ is a sinusoid with amplitude $\mathbf{A}$, angular frequency $\Omega$ (radians/s) and phase offset $\Theta$ (radians). The signal $s(t)$ received at the center of the antenna array for a bearing $\phi$ is given by:

$$
s(t)=A \cos (\Omega t+\Theta)
$$




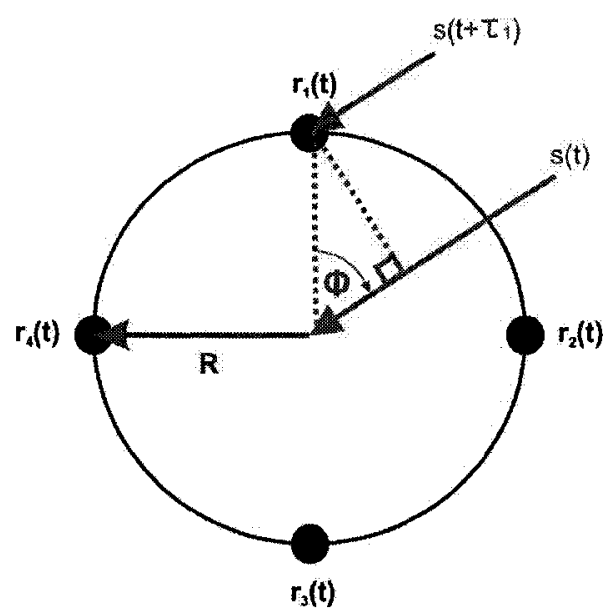

Figure 2.1: The circular antenna array and one signal arriving from a bearing $\phi$.

The distance between the transmitter and the antenna array is assumed to be much greater than the antenna radius (>100 times). Therefore, the received signal can be treated as a plane wave incident on the antenna array. The signal $\mathrm{s}_{x}(\mathrm{t})$, measured at the $\mathbf{x}^{\text {th }}$ antenna, is a time shifted version of $\mathbf{s}(\mathbf{t})$ and is given by:

$$
s_{x}(t)=A \cos \left[\Omega\left(t+\tau_{x}\right)+\Theta\right]
$$

where the time shift $\tau_{x}$ is given by:

$$
\tau_{x}=\frac{R \cos \left(\phi-(x-1) \frac{\pi}{2}\right)}{c}
$$

The initial signal phase $\Theta$ and the time shift $\tau_{x}$ represent a combined phase $\Theta_{x}$, given by:

$$
\Theta_{x}=\Theta+\left(\frac{\Omega R \cos \left(\phi-(x-1) \frac{\pi}{2}\right)}{c}\right)
$$

Substituting equation 2.4 into equation 2.2 , the signal arriving at the $\mathbf{x}^{\text {th }}$ antenna 
becomes:

$$
s_{x}(t)=A \cos \left(\Omega t+\Theta_{x}\right)
$$

In general, multiple sinusoids $(\mathbf{M})$, can be received by the antenna array, with independent amplitude $\left(A_{m}\right)$, frequency $\left(\Omega_{m}\right)$, phase $\left(\Theta_{m}\right)$, and bearing $\left(\phi_{m}\right)$. The phase and bearing of the sinusoids is assumed to be uniformly distributed between 0 and $2 \pi$. The $m^{t h}$ sinusoid on the $\mathbf{x}^{t h}$ antenna is given by:

$$
s_{x, m}(t)=A_{m} \cos \left(\Omega_{m} t+\Theta_{x, m}\right)
$$

Additionally, the measurements are corrupted by AWGN. The noise received on the $\mathbf{x}^{\text {th }}$ antenna, denoted $\eta_{x}$, is zero mean AWGN with variance $\sigma^{2}$ and probability density function given by equation 2.7 .

$$
p_{\eta_{x}}(x)=\frac{1}{\sqrt{(2 \pi)} \sigma} e^{-\frac{x^{2}}{2 \sigma^{2}}}
$$

The noise is assumed to be independent of $\mathbf{s}_{x, m}(\mathbf{t})$ and of the noise at the other antennas. It is assumed that the noise variance $\sigma^{2}$, is the same at each antenna. The generalized equation for the received signal on the $\mathbf{x}^{\text {th }}$ antenna is given by:

$$
r_{x}(t)=\sum_{m=1}^{M} s_{x, m}(t)+\eta_{x}(t)
$$

\subsubsection{The Baseband Signal}

The received signal is tuned to baseband prior to analog to digital conversion and digital signal processing. A quadrature demodulator, illustrated in Figure 2.2, is used to tune the received signals to baseband. Each receiver channel implements a 
quadrature demodulator and the $\mathbf{X}$ channels are assumed to be phase coherent.

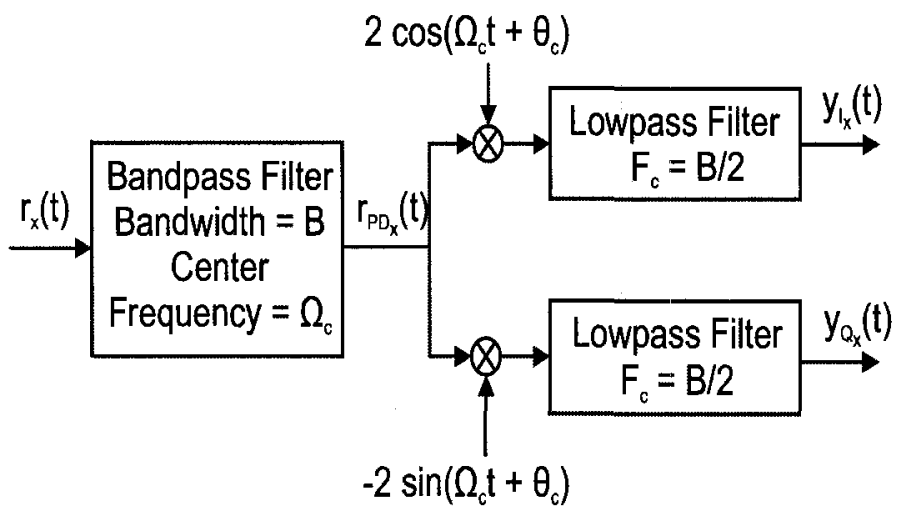

Figure 2.2: Each receiver tunes the signals to baseband with a narrowband quadrature demodulator

The first component of the narrowband quadrature demodulator is the predetection Intermediate Frequency (IF) filter. The predetection filter is a bandpass filter with bandwidth $\mathbf{B}$ centered at $\Omega_{c}$, the carrier frequency of the demodulator. The spectrum of the received signal at antenna $\mathbf{x}$ is illustrated in Figure 2.3 for the case of a single sinusoid plus AWGN, with a power spectral density of $\frac{N_{0}}{2}$.

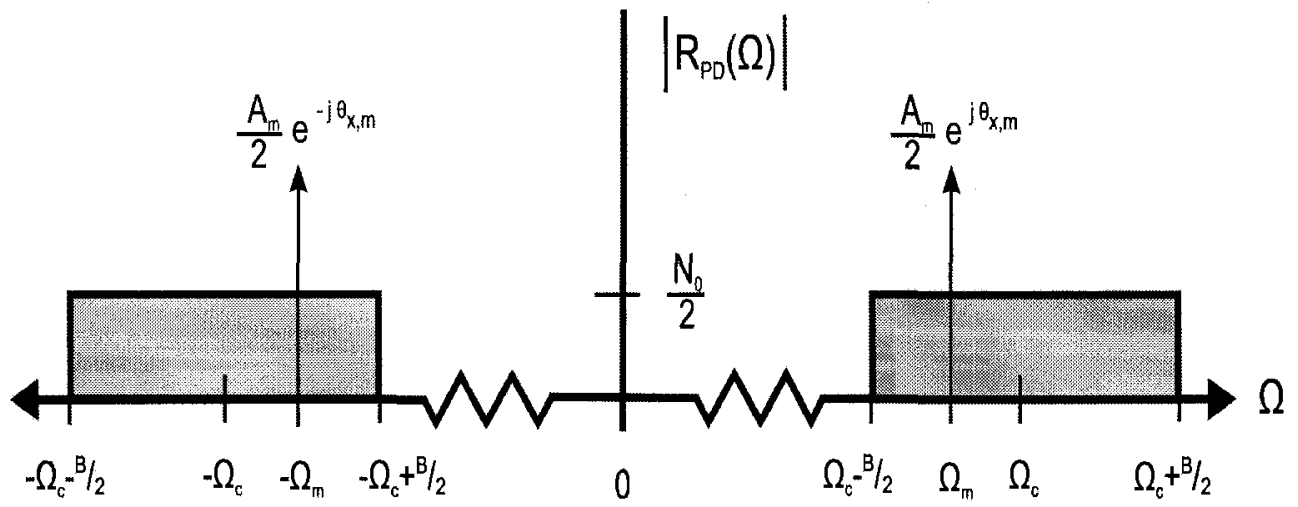

Figure 2.3: The spectrum of the predetection signal

The SNR of the received signal is a key parameter in signal detection and estimation performance. The signal to noise ratio for the predetection signal $\mathbf{S N R}_{P D_{x}}$ 
is the ratio of the predetection signal power $\mathbf{P}_{s_{P D_{x}}}$ relative to the predetection noise power $\mathbf{P}_{\eta_{x}}$. For the case of a single sinusoid, $\mathbf{S N R}_{P D_{x}}$ is given by:

$$
\begin{aligned}
S N R_{P D_{x}} & =\frac{P_{s_{P D_{x}}}}{P_{\eta_{x}}} \\
& =\frac{\frac{1}{T} \int_{0}^{T}\left|\left[A_{m} \cos \left(\Omega_{m} t+\Theta_{x, m}\right)\right]\right|^{2} d t}{\sigma_{P D_{x}}^{2}} \\
& =\frac{\frac{A_{m}^{2}}{2}}{2\left(\frac{N_{0}}{2}\right) B} \\
& =\frac{A_{m}^{2}}{2 N_{0} B}
\end{aligned}
$$

where the term $\mathbf{T}$ represents the period.

To illustrate the tuning process, the noise term $\eta_{P D_{x}}$ is separated into its in-phase and quadrature components, producing:

$$
r_{P D_{x}}(t)=\sum_{m=1}^{M} s_{x, m}(t)+\eta_{I_{x}}(t) \cos \left(\Omega_{c} t+\Theta_{c}\right)-\eta_{Q_{x}}(t) \sin \left(\Omega_{c} t+\Theta_{c}\right)
$$

where,

$$
\overline{\eta_{P D_{x}}^{2}(t)}=\frac{1}{2} \overline{\eta_{I_{x}}^{2}(t)}+\frac{1}{2} \overline{\eta_{Q_{x}}^{2}(t)}
$$

and $\Theta_{c}$ represents the phase of the carrier.

The predetection signal $\mathbf{r}_{P D_{x}}(\mathbf{t})$ is mixed to baseband and filtered with a lowpass filter to remove the double frequency terms. The resulting baseband signal $\mathbf{y}_{B B_{x}}(\mathbf{t})$ is complex, consisting of an in-phase $\mathbf{y}_{I_{x}}(\mathbf{t})$ component and a quadrature $\mathbf{y}_{Q_{x}}(\mathbf{t})$ component. 
The in-phase component is given by:

$$
\begin{aligned}
y_{I_{x}}(t)= & \operatorname{LPF}\left[r_{B P_{x}}(t)\left(2 \cos \left(\Omega_{c} t+\Theta_{c}\right)\right)\right] \\
= & \operatorname{LPF}\left[\left(\sum_{m=1}^{M} A_{m} \cos \left(\Omega_{m} t+\Theta_{x, m}\right)+\eta_{I_{x}}(t) \cos \left(\Omega_{c} t+\Theta_{c}\right)\right.\right. \\
& \left.\left.-\eta_{Q_{x}}(t) \sin \left(\Omega_{c} t+\Theta_{c}\right)\right)\left(2 \cos \left(\Omega_{c} t+\Theta_{c}\right)\right)\right] \\
= & \sum_{m=1}^{M} A_{m} \cos \left(\left(\Omega_{m}-\Omega_{c}\right) t+\left(\Theta_{x, m}-\Theta_{c}\right)\right)+\eta_{I_{x}}(t) \\
= & \sum_{m=1}^{M} s_{I_{x, m}}+\eta_{I_{x}}(t)
\end{aligned}
$$

and the quadrature component is given by:

$$
\begin{aligned}
y_{Q_{x}}(t)= & \operatorname{LPF}\left[r_{B P_{x}}(t)\left(-2 \sin \left(\Omega_{c} t+\Theta_{c}\right)\right)\right] \\
= & \operatorname{LPF}\left[\left(\sum_{m=1}^{M} A_{m} \cos \left(\Omega_{m} t+\Theta_{x, m}\right)+\eta_{I_{x}}(t) \cos \left(\Omega_{c} t+\Theta_{c}\right)\right.\right. \\
& \left.\left.-\eta_{Q_{x}}(t)(t) \sin \left(\Omega_{c} t+\Theta_{c}\right)\right)\left(-2 \sin \left(\Omega_{c} t+\Theta_{c}\right)\right)\right] \\
= & \sum_{m=1}^{M} A_{m} \sin \left(\left(\Omega_{m}-\Omega_{c}\right) t+\left(\Theta_{x, m}-\Theta_{c}\right)\right)+\eta_{Q_{x}}(t) \\
= & \sum_{m=1}^{M} s_{Q_{x, m}}+\eta_{Q_{x}}(t)
\end{aligned}
$$

The spectrum of the in-phase baseband component is illustrated in Figure 2.4: 


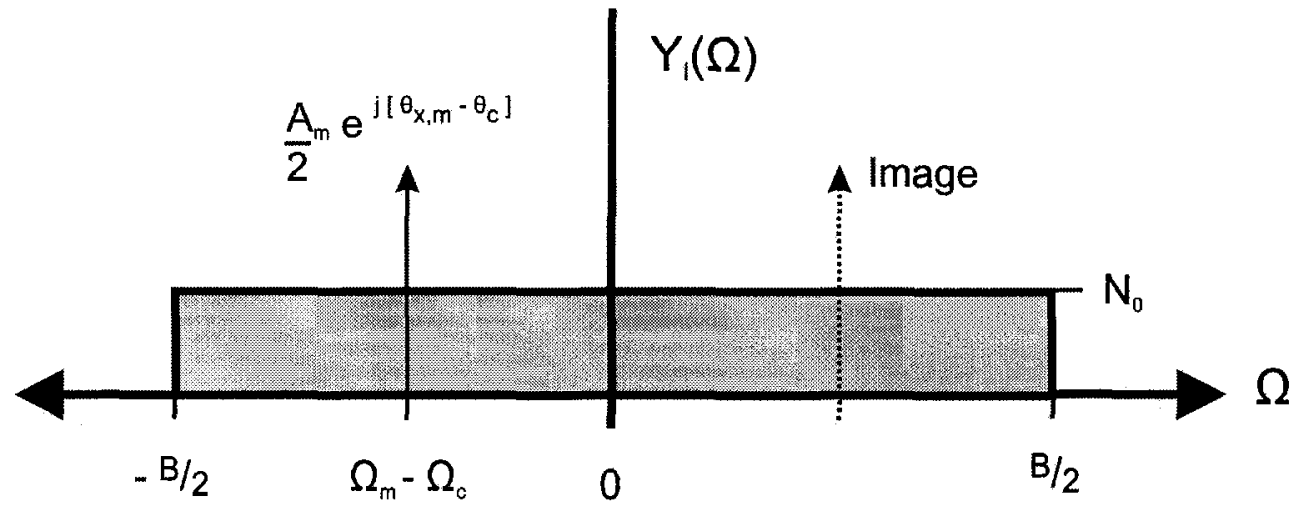

Figure 2.4: The spectrum of the received signal after tuning to baseband

Therefore, the complex baseband signal $\mathbf{y}_{B B_{x}}(\mathrm{t})$ is:

$$
\begin{aligned}
y_{B B_{x}}(t)= & y_{I_{x}}(t)+j y_{Q_{x}}(t) \\
= & \sum_{m=1}^{M} A_{m} \cos \left(\left(\Omega_{m}-\Omega_{c}\right) t+\left(\Theta_{x, m}-\Theta_{c}\right)\right)+\eta_{I_{x}}(t) \\
& +j \sum_{m=1}^{M} A_{m} \sin \left(\left(\Omega_{m}-\Omega_{c}\right) t+\left(\Theta_{x, m}-\Theta_{c}\right)\right)+j \eta_{Q_{x}}(t) \\
= & \sum_{m=1}^{M} A_{m} e^{j\left[\left(\Omega_{m}-\Omega_{c}\right) t+\left(\Theta_{x, m}-\Theta_{c}\right)\right]}+\left[\eta_{I_{x}}(t)+j \eta_{Q_{x}}(t)\right] \\
= & \sum_{m=1}^{M} s_{B B_{x, m}}(t)+\eta_{B B_{x}}(t)
\end{aligned}
$$

The baseband signal to noise ratio $\mathbf{S N R}_{B B_{x}}$ is the ratio of the baseband signal power $\mathbf{P}_{s_{B B x}}$ relative to the baseband noise power $\mathbf{P}_{\eta_{B B_{x}}}$. For the case of a single 
sinusoid, $\mathbf{S N R}_{B B_{x}}$ is given by:

$$
\begin{aligned}
S N R_{B B_{x}} & =\frac{P_{s_{B B_{x}}}}{P_{\eta_{B B_{x}}}} \\
& =\frac{\frac{1}{T} \int_{0}^{T}\left|\left[A_{m} e^{j\left[\left(\Omega_{m}-\Omega_{c}\right) t+\left(\Theta_{x, m}-\Theta_{c}\right)\right]}\right]\right|^{2} d t}{\sigma_{I_{x}}^{2}+\sigma_{Q_{x}}^{2}} \\
& =\frac{A_{m}^{2}}{2 N_{0} B} \\
& =S N R_{P D_{x}}
\end{aligned}
$$

\subsubsection{The Digital Baseband Signal}

The continuous-time baseband signal $\mathrm{s}_{B B_{x}}(\mathrm{t})$ is converted to discrete-time with an Analog to Digital Converter (ADC). The ADC uniformly samples the in-phase and quadrature components of the baseband signal, at a rate $\mathbf{T}_{S}$, producing:

$$
\begin{aligned}
y_{I_{x}}[n] & =y_{I_{x}}\left(n T_{s}\right) \\
& =\sum_{m=1}^{M} A_{m} \cos \left(\left(\Omega_{m}-\Omega_{c}\right) n T_{S}+\left(\Theta_{x, m}-\Theta_{c}\right)\right)+\eta_{I_{x}}\left(n T_{S}\right) \\
& =\sum_{m=1}^{M} A_{m} \cos \left(\omega_{m} n+\theta_{x, m}\right)+\eta_{I_{x}}[n] \\
& =\sum_{m=1}^{M} s_{I_{x, m}}[n]+\eta_{I_{x}}[n]
\end{aligned}
$$


and

$$
\begin{aligned}
y_{Q_{x}}[n] & =y_{Q_{x}}\left(n T_{s}\right) \\
& =\sum_{m=1}^{M} A_{m} \sin \left(\left(\Omega_{m}-\Omega_{c}\right) n T_{S}+\left(\Theta_{x, m}-\Theta_{c}\right)\right)+\eta_{Q_{x}}\left(n T_{S}\right) \\
& =\sum_{m=1}^{M} A_{m} \sin \left(\omega_{m} n+\theta_{x, m}\right)+\eta_{Q_{x}}[n] \\
& =\sum_{m=1}^{M} s_{Q_{x, m}}[n]+\eta_{Q_{x}}[n]
\end{aligned}
$$

where the normalized angular frequency $\omega_{m}$ (radians) is given by:

$$
\omega_{m}=\left(\Omega_{m}-\Omega_{c}\right) T_{S}
$$

and the consolidated phase term $\theta_{x, m}$ (radians) is given by:

$$
\theta_{x, m}=\Theta_{x, m}-\Theta_{c}
$$

Therefore, the digital complex baseband signal $\mathbf{y}_{B B_{x}}[\mathbf{n}]$ is:

$$
\begin{aligned}
y_{B B_{x}}[n] & =\sum_{m=1}^{M} A_{m} e^{j\left[\omega m n+\theta_{x}, m\right]}+\eta_{B B_{x}}[n] \\
& =\sum_{m=1}^{M} s_{B B_{x, m}}[n]+\eta_{B B_{x}}[n]
\end{aligned}
$$

where the noise component is Complex White Gaussian Noise (CWGN) with distribution:

$$
\eta_{B B_{x}} \sim N\left(0, \sigma_{z}^{2}\right)
$$


where

$$
\sigma_{I}^{2}=\sigma_{Q}^{2}=\frac{\sigma_{z}^{2}}{2}
$$

The spectrum of the discrete-time spectrum for the in-phase baseband signal is illustrated in figure 2.5:

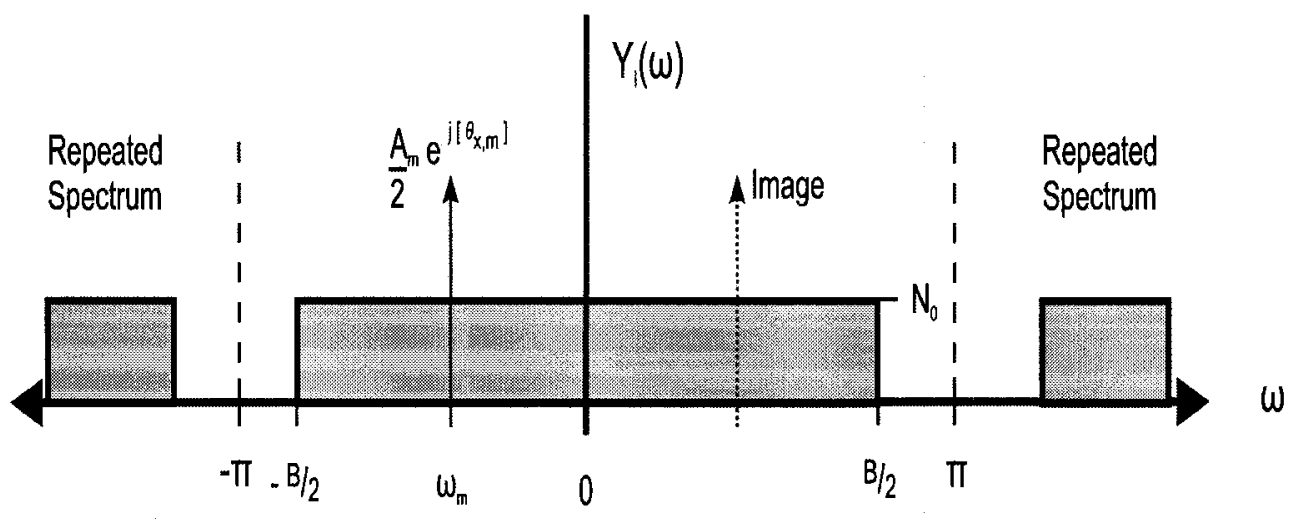

Figure 2.5: The discrete-time spectrum of the in-phase baseband signal.

\subsection{Sinusoidal Parameter Estimation}

Sinusoidal parameter estimation in the presence of white Gaussian noise is a classical problem. For single tone and reasonably spaced sinusoids, the traditional DFT based spectral estimator is a computationally efficient and effective method for sinusoidal parameter estimation. However, for closely spaced sinusoids (frequency separation $<\frac{1}{N}$ ), the DFT based spectral estimator leads to a nonlinear least squares problem and high resolution spectral estimation techniques must be employed [7], [2].

High resolution techniques such as Auto-Regressive Moving Average (ARMA) modeling, MUltiple SIgnal Classification (MUSIC) and Estimation of Signal Parameter via Rotational Invariance Techniques (ESPRIT) perform well in high SNR environments. However, in low SNR environments, these techniques tend to break down 
[7]. Within the class of high resolution spectral estimation techniques, the eigendecomposition technique provides greater accuracy under low SNR conditions; however, the computational complexity can be prohibitive for practical applications. The sinusoidal signals targeted in this thesis are reasonably spaced ( $\geq 4$ frequency bins);

therefore, the traditional DFT based spectral estimator is an efficient estimator[8] and will be studied in this thesis.

Classical sinusoidal parameter estimation is typically a two step process involving a coarse estimation stage followed by a fine estimation stage [9]. The coarse estimation stage is typically Fourier Transform based, due to the computational efficiency of the FFT. The fine estimation stage is typically performed via interpolation, zero padding or alternative methods of higher precision frequency estimation.

The following sections describe the performance of the FFT as a coarse frequency estimator for single and reasonably spaced sinusoids. It will be shown that an FFT based technique is a close approximation to a MLE for single and reasonably spaced sinusoids.

\subsubsection{Performance Bounds For Sinusoidal Parameter Esti- mation}

In parameter estimation, performance bounds are used to indicate the maximum achievable performance for a given signal type. Estimator bias and Root Mean Square Error (RMSE) are typically used to quantify estimator performance.

If the bias is minimized, the CRLB provides a theoretical limit on the minimum variance of an estimator. Additionally, the CRLB indicates the asymptotically optimum performance of a MLE. Therefore, the results of the CRLB analysis will be used to define a MLE for multiple sinusoids with unknown frequency, amplitude and 
phase, for signals above threshold. The CRLB is given by the inverse of the Fisher Information Matrix $(\mathbf{J}\{\alpha\})$. The CRLB is discussed in greater detail in Appendix A, where the vector CRLB equation is given by:

$$
\operatorname{var}\left\{\hat{\alpha}_{i}\right\} \geq \frac{1}{\mathbf{J}\{\alpha\}}=\left(-E_{p(z ; \alpha)}\left\{\frac{\partial^{2} \ln \mathbf{p}(\mathbf{z} ; \alpha)}{\partial \alpha^{2}}\right\}\right)^{-1}
$$

and $\mathrm{p}(\mathrm{z} ; \alpha)$ is the joint probability density function for the sample vector $y_{z}[n]$ with unknown parameter vector $\alpha$. The diagonal elements of equation 2.32 indicate the CRLB for the individual elements of $\alpha$.

A complete analysis of performance bounds for sinusoidal estimation is presented by Rife and Boorstyn [10], [8]. The specific case of sinusoids with unknown frequency, amplitude and phase is presented in the following sections. The performance bounds are evaluated first for the single tone case. The results of the CRLB calculation are then used to identify a realization of a MLE for a single sinusoid in AWGN. In Section 2.2.4, the results are generalized for the case of multiple reasonably spaced sinusoids, with all parameters unknown.

\subsubsection{Performance Bounds for Single Sinusoid Estimation}

The equation for a single complex sinusoid corrupted by AWGN is given by:

$$
\begin{aligned}
y_{z}[n] & =y_{I}[n]+j y_{Q}[n] \\
& =A \cos (\omega n+\theta)+\eta_{I}[n]+j\left(A \sin (\omega n+\theta)+\eta_{Q}[n]\right) \\
& =A e^{j(\omega n+\theta)}+\eta_{z}[n]
\end{aligned}
$$

where

$$
n=0, \ldots, N-1
$$


and the unknown parameter vector is given by [11]:

$$
\alpha=[\omega, A, \theta]^{T}
$$

The $\eta_{I}[n]$ and $\eta_{Q}[n]$ are independently identically distributed (i.i.d.) white Gaussian noise sources with distribution $\mathrm{N}\left(0, \sigma^{2}\right)$. Therefore, $\eta_{z}[n]$ is CWGN, with distribution $\mathrm{N}\left(0, \sigma_{z}^{2}\right)$, where $\sigma_{z}=\sqrt{2} \sigma$.

The probability density function for $y_{z}[n]$ is given by:

$$
p\left(y_{z} ; \alpha\right)=\left(\frac{1}{\pi \sigma_{z}^{2}}\right)^{N} e^{-\frac{1}{\sigma_{z}^{2}} \sum_{n=0}^{N-1}\left|y_{z}[n]-\mu_{z}[n]\right|^{2}}
$$

where $\mu_{z}[n]=A e^{j(\omega n+\theta)}$.

The probability density function is substituted into equation 2.32 , to determine the CRLBs for the unknown parameters. The elements of the Fisher information matrix are given by:

$$
\mathbf{J}\left\{\alpha_{i j}\right\}=\frac{2}{\sigma_{z}^{2}} \operatorname{Re}\left\{\sum_{n=0}^{N-1} \frac{\partial \mu_{z}^{*}[n] \partial \mu_{z}[n]}{\partial \alpha_{i} \partial \alpha_{j}}\right\}
$$

Substituting $\mu_{z}[n]$ and solving the partial differential equation, the Fisher information matrix (for unknown frequency, amplitude and phase) is given by:

$$
\mathbf{J}\{\alpha\}=\frac{2}{\sigma_{z}^{2}}\left[\begin{array}{ccc}
A^{2} \sum_{n=0}^{N-1} n^{2} & 0 & A^{2} \sum_{n=0}^{N-1} n \\
0 & N & 0 \\
A^{2} \sum_{n=0}^{N-1} n & 0 & A^{2} N
\end{array}\right]
$$


The inverse of the Fisher information matrix is then:

$$
\frac{1}{\mathbf{J}\{\alpha\}}=\left[\begin{array}{ccc}
\frac{6}{\operatorname{snr} N\left(N^{2}-1\right)} & 0 & \frac{-3}{\operatorname{snr} N(N+1)} \\
0 & \frac{\sigma_{z}^{2}}{2 N} & 0 \\
\frac{-3}{\operatorname{snr} N(N+1)} & 0 & \frac{2 N-1}{\operatorname{snr} N(N+1)}
\end{array}\right]
$$

where snr is the signal to noise ratio given by:

$$
s n r=\frac{A^{2}}{\sigma_{z}^{2}}
$$

The diagonal elements of $\mathbf{J}^{-1}\{\alpha\}$ indicate the CRLBs for the unknown parameter vector $\alpha=[\omega, \mathrm{A}, \theta]^{T}$. Therefore:

$$
\begin{gathered}
\operatorname{var}(\hat{\omega}) \geq \frac{6}{\operatorname{snr} N\left(N^{2}-1\right)} \\
\operatorname{var}(\hat{A}) \geq \frac{\sigma_{z}^{2}}{2 N}
\end{gathered}
$$

and

$$
\operatorname{var}(\hat{\theta}) \geq \frac{2 N-1}{\operatorname{snr} N(N+1)}
$$

\subsubsection{Maximum Likelihood Estimation for a Single Sinusoid}

The maximum likelihood estimate of $\alpha$, denoted $\hat{\alpha}$, maximizes the likelihood function $\mathrm{p}(\mathrm{z} ; \alpha)$, for the observed sample vector $y_{z}[n][12]$. The estimate $\hat{\alpha}$ also maximizes the log-likelihood function:

$$
\ln p\left(y_{z} ; \alpha\right)=-\ln \left[\left(\pi \sigma_{z}^{2}\right)^{N}\right]-\frac{1}{\sigma_{z}^{2}} \sum_{n=0}^{N-1}\left|y_{z}[n]-\mu_{z}[n]\right|^{2}
$$


With some manipulation, Rife [10] has developed an alternative representation:

$$
L_{0}=\left(\ln p\left(y_{z} ; \alpha\right)+\ln \left[\left(\pi \sigma_{z}^{2}\right)^{N}\right]\right)\left(\frac{\sigma_{z}^{2}}{N}\right)=-\frac{1}{N} \sum_{n=0}^{N-1}\left|y_{z}[n]-\mu_{z}[n]\right|^{2}
$$

The $\left|y_{z}[n]\right|^{2}$ terms are constants and can be dropped from the equation yielding:

$$
\begin{aligned}
L & =\frac{1}{N} \sum_{n=0}^{N-1}\left(y_{z}^{*}[n] \mu_{z}[n]+y_{z}[n] \mu_{z}^{*}[n]\right)-\frac{1}{N} \sum_{n=0}^{N-1}\left(\mu_{z}^{*}[n] \mu_{z}[n]\right) \\
& =\frac{2}{N} \sum_{n=0}^{N-1} \operatorname{Re}\left\{\left(y_{z}[n] \mu_{z}^{*}[n]\right)\right\}-\frac{1}{N} \sum_{n=0}^{N-1}\left|\mu_{z}[n]\right|^{2} \\
& =\frac{2}{N} \operatorname{Re}\left\{\sum_{n=0}^{N-1}\left(y_{z}[n] A e^{-j(\omega n+\theta)}\right)\right\}-\frac{1}{N} \sum_{n=0}^{N-1} A^{2} \\
& =\frac{2 A}{N} \operatorname{Re}\left\{e^{-j \theta} Z\left(e^{j \omega}\right)\right\}-A^{2}
\end{aligned}
$$

where

$$
Z\left(e^{j \omega}\right)=\sum_{n=0}^{N-1} y_{z}[n] e^{-j \omega n}
$$

and $\theta$ is assumed to be uniformly distributed over $[0,2 \pi)$. The $\log$-likelihood function is maximum for $\theta=\arg \left(Z\left(e^{j \omega}\right)\right)$, therefore:

$$
\max _{\theta} L=\frac{2 A}{N}\left|Z\left(e^{j \omega}\right)\right|-A^{2}
$$

Let $\hat{\omega}$ be the value that maximizes $\left|Z\left(e^{j \omega}\right)\right|$ then,

$$
\max _{\theta \omega} L=\frac{2 A}{N}\left|Z\left(e^{j \hat{\omega}}\right)\right|-A^{2}
$$


Taking the derivative with respect to $\mathrm{A}$ and setting the result to zero, the estimate $\hat{A}$ is given by:

$$
\hat{A}=\frac{1}{N}\left|Z\left(e^{j \hat{\omega}}\right)\right|
$$

Substituting $\hat{A}$ into equation 2.47 the maximum likelihood result is:

$$
\max _{\theta \omega A} L=\frac{1}{N^{2}}\left|Z\left(e^{j \hat{\omega}}\right)\right|^{2}=\frac{1}{N^{2}}\left|\sum_{n=0}^{N-1} y_{z}[n] e^{-j \hat{\omega} n}\right|^{2}
$$

Therefore, the values $\hat{A}$ and $\hat{\omega}$ are the MLEs of A and $\omega$, while the maximum likelihood estimate of $\theta$ is:

$$
\hat{\theta}=\arg \left\{Z\left(e^{j \hat{\omega}}\right)\right\}
$$

The function $\mathrm{Z}\left(\mathrm{e}^{j \omega}\right)$ is periodic in $\omega$ with a period $\omega_{s}=2 \pi / T$. Additionally, the discrete components of $\mathrm{Z}\left(\mathrm{e}^{j \omega}\right)$ are given by:

$$
Z[k]=\sum_{n=0}^{N-1} y_{z}[n] e^{-\frac{j 2 \pi n k}{N}}
$$

which is the Discrete Fourier Transform of the input data. Furthermore,

$$
\begin{aligned}
|Z[k]|^{2} & =\frac{1}{N^{2}}\left|\sum_{n=0}^{N-1} y_{z}[n] e^{-j \hat{\omega} n}\right|^{2} \\
& =\frac{1}{N} P[k]
\end{aligned}
$$

is the equation of the periodogram (equation B.14) scaled by a constant $1 / N$. Therefore, the DFT based periodogram is a realization of the ML estimator for a single sinusoid with all parameters unknown in the presence of AWGN [10], [7], [9]. 


\subsubsection{Performance Bounds for Multiple Sinusoids}

The equation for multiple complex sinusoids corrupted by AWGN is given by:

$$
\begin{aligned}
y_{z}[n] & =y_{I}[n]+j y_{Q}[n] \\
& =\sum_{m=1}^{M} A_{m} \cos \left(\omega_{m} n+\theta_{m}\right)+\eta_{I}[n]+j\left(\sum_{m=1}^{M} A_{m} \sin \left(\omega_{m} n+\theta_{m}\right)+\eta_{Q}[n]\right) \\
& =\sum_{m=1}^{M} A_{m} e^{j\left(\omega_{m} n+\theta_{m}\right)}+\eta_{z}[n]
\end{aligned}
$$

For the case of multiple sinusoids, $\mu_{z}[\mathrm{n}]=\sum_{m=1}^{M} A_{m} e^{j\left(\omega_{m} n+\theta_{m}\right)}$, the likelihood equation becomes [8]:

$$
\begin{aligned}
L= & \frac{1}{N} \sum_{n=0}^{N-1}\left(y_{z}^{*}[n] \mu_{z}[n]+y_{z}[n] \mu_{z}^{*}[n]\right)-\frac{1}{N} \sum_{n=0}^{N-1}\left(\mu_{z}^{*}[n] \mu_{z}[n]\right) \\
= & \frac{1}{N} \sum_{n=0}^{N-1}\left(y_{z}^{*}[n] \sum_{m=1}^{M} A_{m} e^{j\left(\omega_{m} n+\theta_{m}\right)}+y_{z}[n] \sum_{m=1}^{M} A_{m} e^{-j\left(\omega_{m} n+\theta_{m}\right)}\right) \\
& -\frac{1}{N} \sum_{n=0}^{N-1}\left(\sum_{m=1}^{M} A_{m} e^{-j\left(\omega_{m} n+\theta_{m}\right)}\right)\left(\sum_{m=1}^{M} A_{m} e^{j\left(\omega_{m} n+\theta_{m}\right)}\right) \\
= & \sum_{m=1}^{M}\left(\frac{2 A_{m}}{N} \operatorname{Re}\left\{\sum_{n=0}^{N-1} y_{z}[n] e^{-j\left(\omega_{m} n+\theta_{m}\right)}\right\}\right) \\
& -\frac{1}{N} \sum_{n=0}^{N-1}\left(\sum_{m=1}^{M} A_{m} e^{-j\left(\omega_{m} n+\theta_{m}\right)}\right)\left(\sum_{i=1}^{M} A_{m} e^{j\left(\omega_{m} n+\theta_{m}\right)}\right) \\
= & \sum_{m=1}^{M}\left(\frac{2 A_{m}}{N} R e\left\{e^{-j \theta_{m}} Z\left(e^{j \omega_{m}}\right)\right\}-A_{m}^{2}\right) \\
& -\frac{1}{N} \sum_{m=1}^{M} \sum_{\substack{i=1 \\
i \neq m}}^{M} A_{m} A_{i} \sum_{n=0}^{N-1} e^{-j\left(\omega_{m}-\omega_{i}\right) n+\left(\theta_{m}-\theta_{i}\right)}
\end{aligned}
$$

This likelihood equation is difficult to maximize and the resulting estimator would be computationally expensive. Also of note, when $\mathrm{M}=1$, the second term of equation 
2.55 vanishes. Additionally, Rife and Boorstyn [8] have shown that for large $\mathrm{N}$ and $\mathrm{M}>1$, the magnitude of the second term is relatively small and can be dropped, resulting in an "almost MLE". The approximate likelihood equation is given by:

$$
L_{\text {approx }}=\sum_{m=1}^{M}\left(\frac{2 A_{m}}{N} \operatorname{Re}\left\{e^{-j \theta_{m}} Z\left(e^{j \omega_{m}}\right)\right\}-A_{m}^{2}\right)
$$

As with the single tone case, the frequency estimates $\hat{\omega}_{m}$ maximize $\left|Z\left(e^{j \omega}\right)\right|$. The corresponding amplitude and phase estimates are provided by the DFT and are given by:

$$
\hat{A}_{m}=\frac{1}{N}\left|Z\left(e^{j \hat{\omega}_{m}}\right)\right|
$$

and

$$
\hat{\theta}_{m}=\arg \left\{Z\left(e^{j \hat{\omega}_{m}}\right)\right\}
$$

The impact of dropping the cross-product terms in equation 2.55 is a bias in the frequency and amplitude estimates, $\hat{\omega}_{m}$ and $\hat{A}_{m}$ respectively. Neglecting the crossproduct terms causes sidelobes in the spectral response, biasing the spectral estimates from their true values. The sidelobe behavior observed in the DFT output is commonly referred to as spectral leakage and the distortion of one signal is due to the displaced energy of another signal present.

The spectral leakage is caused by finite length of the input signal, which can be interpreted as the rectangular windowing of an infinite length signal. The rectangular window produces a sinc response in the frequency domain, given by:

$$
\operatorname{sinc}(x)=\frac{\sin (\pi x)}{\pi x}
$$


Spectral leakage can be minimized using window functions. When a window function is combined with the FFT, the process is referred to as a modified FFT. Multiple window functions exist, as discussed in Appendix B. Several tradeoff's are made based on performance parameters such as level of highest sidelobe, mainlobe width, decay rate, etc. The window function should be selected based on the input signal characteristics, primarily relative signal strength, frequency separation and SNR. A common window function in spectral estimation applications is the Hann, and it will be further investigated in this thesis.

When multiple sinusoids are present, window functions increase the frequency estimation accuracy for the FFT; however, there is a penalty associated with this performance increase. Window functions increase the mainlobe width and the variance of the frequency and amplitude estimates [13]. The common technique for reducing the variance is DFT averaging. The Bartlett and Welch periodogram methods, discussed in detail in Appendix B, reduce the variance of the spectral estimates and the performance of these techniques is investigated in this thesis.

The CRLBs and associated ML estimator for multiple sinusoids, with averaged modified periodograms is difficult to compute [9]. For most cases, high resolution techniques should be utilized to resolve closely spaced sinusoids. However, Rife and Boorstyn [8] have shown that when the minimum frequency separation of the sinusoids is greater than four divided by the FFT length and the sample period (4/NT or 4 frequency bins) and an appropriate window function is used, the FFT based coarse estimator is a good approximation of the ML estimator. Under these conditions, the parameter estimates of each of the individual sinusoids approach that of the single sinusoid case.

This is a very useful result for the estimation of multiple weak sinusoids. When 
the minimum frequency separation of the input signal is known, the FFT based estimator can be designed to ensure a minimum separation of 4 FFT bins. In doing so, the superior performance of the FFT relative to high resolution techniques in low SNR conditions [7] can be exploited, and the much simpler single tone CRLBs can be employed as effective performance bounds.

\subsection{Sinusoid Detection Theory}

Signal detection theory is the field of spectral analysis which uses statistical hypothesis testing to determine the detection of a signal of interest in noise.

In the binary hypothesis problem, the received data is analyzed and a decision is made between the null hypothesis $\left(\mathcal{H}_{0}\right)$ and the alternative hypothesis $\left(\mathcal{H}_{1}\right)$ :

$$
\begin{array}{ll}
\mathcal{H}_{0}: y[n]=\eta[n] & n=0,1, \ldots, N-1 \\
\mathcal{H}_{1}: y[n]=s[n]+\eta[n] & n=0,1, \ldots, N-1
\end{array}
$$

Since the noise component is a random variable, the detection problem must decide which of the two random processes occurred, based on their Probability Density Function (PDF). A detection is decided when the parameters of the observed data exceed the detection threshold.

Several errors and conditions are of particular importance in the analysis of detection performance. The probability of false alarm $\left(P_{F A}\right)$ is the probability of detecting

the signal when it is not present. The probability of a miss $\left(P_{M}\right)$ is the probability of not detecting the signal when it is present. The probability of detection $\left(P_{D}\right)$ is the probability of detecting the signal when it is present. The error conditions and terms 
are summarized as follows:

$$
\begin{aligned}
& P_{F A}=p\left(\text { decide } \mathcal{H}_{1} \mid \mathcal{H}_{0} \text { was sent }\right) \\
& P_{M}=p\left(\text { decide } \mathcal{H}_{0} \mid \mathcal{H}_{1} \text { was sent }\right) \\
& P_{D}=p\left(\text { decide } \mathcal{H}_{1} \mid \mathcal{H}_{1} \text { was sent }\right)
\end{aligned}
$$

Typically, detection performance is presented in the form of a ROC diagram. The ROC diagram is a plot of a signal detector's $P_{F A}$ vs. $P_{D}$ for the detection scenario under investigation and its corresponding signal conditions. A sample ROC diagram is illustrated in Figure 2.6.

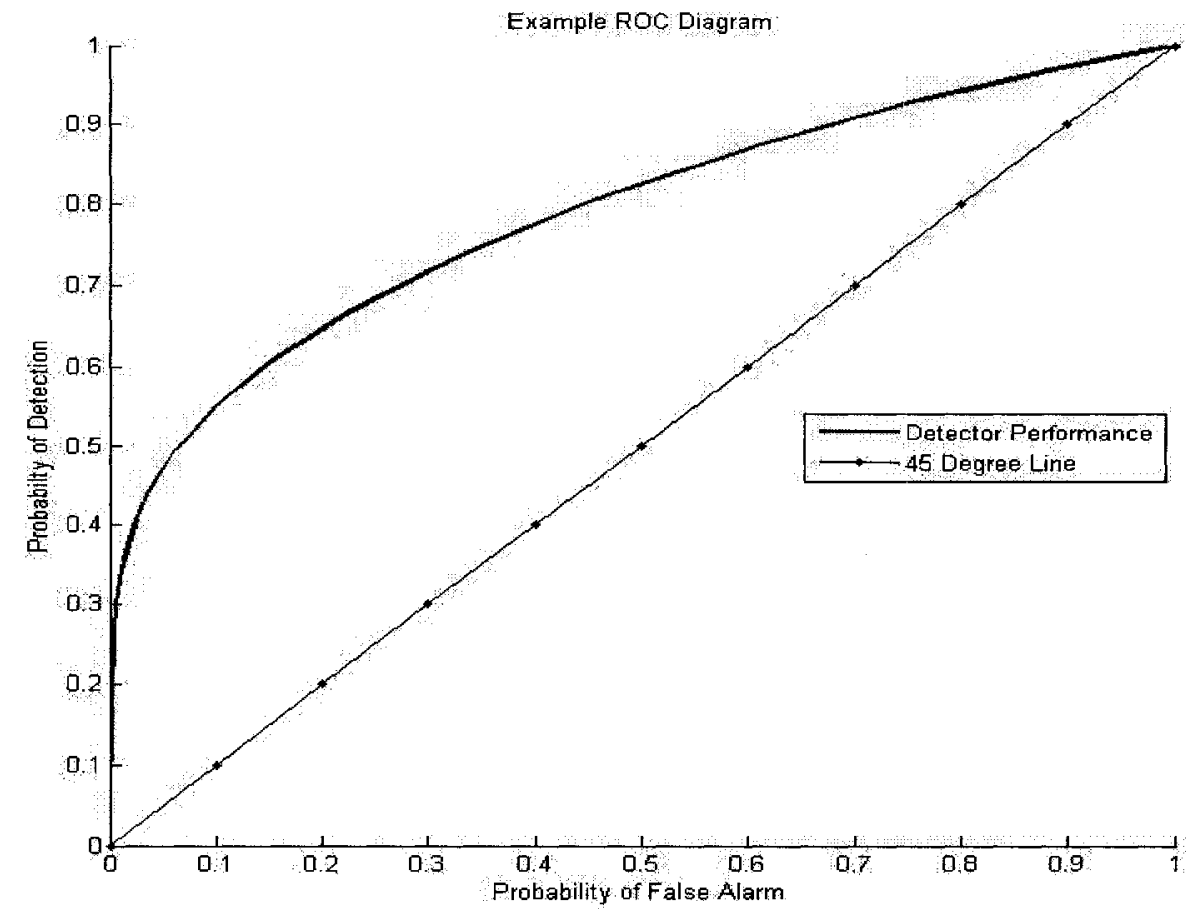

Figure 2.6: Example of a ROC diagram.

The 45 degree line indicates the detection performance for $P_{F A}=P_{D}$, or ignoring the data and deciding a detection by flipping a coin [1]. The ROC curve should always 
be above the 45 degree line.

\subsubsection{Primary Signal Detection Approaches}

The two primary approaches to solving the binary hypothesis problem are the NeymanPearson (NP) theorem and the Bayesian approach.

The NP theorem uses the likelihood ratio test to maximize the probability of detection for a given probability of false alarm. The NP theorem decides $\mathcal{H}_{1}$ if:

$$
L(\mathbf{y})=\frac{p\left(\mathbf{y} \mid \mathcal{H}_{1}\right)}{p\left(\mathbf{y} \mid \mathcal{H}_{0}\right)}>\gamma
$$

where $\gamma$ represents the detection threshold.

The NP theorem makes no assumptions about the prior information of unknown parameters and the detection threshold is based on the false alarm criteria. Kay [1] gives the following expression for the calculation of the NP detection threshold:

$$
P_{F A}=\int_{\mathbf{y}: L(y)>\gamma} p\left(\mathbf{y} \mid \mathcal{H}_{0}\right) d \mathbf{y}=\alpha
$$

The Bayesian approach assigns costs to each type of error and minimizes the overall probability of error. The cost $\mathrm{C}_{a b}$ indicates the cost associated with deciding $\mathcal{H}_{a}$ when $\mathcal{H}_{b}$ is true. The Bayesian detection threshold is based on the prior probabilities of the unknown parameters. The Bayesian detector decides $\mathcal{H}_{1}$ if:

$$
\frac{p\left(\mathbf{y} \mid \mathcal{H}_{1}\right)}{p\left(\mathbf{y} \mid \mathcal{H}_{0}\right)}>\frac{\left(C_{10}-C_{00}\right) p\left(\mathcal{H}_{0}\right)}{\left(C_{01}-C_{11}\right) p\left(\mathcal{H}_{1}\right)}=\gamma
$$

The NP detector is commonly realized as a Generalized Likelihood Ratio Test (GLRT), where the MLE of the unknown parameter vector, denoted $\hat{\alpha}$, are calculated 
and substituted into equation 2.61. The GLRT decides $\mathcal{H}_{1}$ if:

$$
L(\mathbf{y})=\frac{p\left(\mathbf{y} \mid \hat{\alpha}_{1}, \mathcal{H}_{1}\right)}{p\left(\mathbf{y} \mid \hat{\alpha}_{0}, \mathcal{H}_{0}\right)}>\gamma
$$

where $\hat{\alpha}_{a}$ is the unknown parameter vector under $\mathcal{H}_{a}$.

The Bayesian approach requires specification of the prior PDFs for the unknown parameters and multidimensional integration to solve the detection threshold. Due to its less restrictive assumptions and ease of implementation the GLRT is more widely used in signal detection applications [1]. Furthermore, for the problem of detecting multiple sinusoidal signals with unknown parameters, the prior PDFs are not known, suggesting the GLRT is the more appropriate detection strategy.

\subsubsection{The GLRT Detector for Single Complex Sinusoid}

For the case of a single complex sinusoid, the binary hypothesis problem is:

$$
\begin{array}{ll}
\mathcal{H}_{0}: \eta[n] & n=0,1, \ldots, N-1 \\
\mathcal{H}_{1}: y[n]=A e^{j(w n+\theta)}+\eta[n] & n=0,1, \ldots, N-1
\end{array}
$$

where the unknown parameter vector $\alpha$ is given by equation 2.35 .

The GLRT is derived first for the case of unknown amplitude and phase and then generalized to incorporate unknown frequency. 


\section{The GLRT for Unknown Amplitude and Phase}

The PDF for a single complex sinusoid in CWGN, defined in equation 2.36, is substituted into equation 2.64, producing:

$$
L(\mathbf{y})=\frac{\left(\frac{1}{\pi^{N} \sigma_{z}^{2 N}}\right) e^{-\frac{1}{\sigma_{z}^{2}} \sum_{n=0}^{N-1}\left|y_{z}[n]-\mu_{z}[n]\right|^{2}}}{\left(\frac{1}{\pi^{N} \sigma_{z}^{2 N}}\right) e^{-\frac{1}{\sigma_{z}^{2}} \sum_{n=0}^{N-1}\left|y_{z}[n]\right|^{2}}}>\gamma
$$

The logarithm is taken, producing the log likelihood function, and substituting $\left(\mu_{z}[n]=\right.$ $\left.\hat{A} e^{j(w n+\hat{\theta})}\right)$ :

$$
\begin{aligned}
\ln L(\mathbf{y}) & =\frac{1}{\sigma_{z}^{2}} \sum_{n=0}^{N-1}\left(y_{z}^{*}[n] \mu_{z}[n]+y_{z}[n] \mu_{z}^{*}[n]-\mu_{z}^{*}[n] \mu_{z}[n]\right)>\ln \gamma \\
& =\frac{1}{\sigma_{z}^{2}} \sum_{n=0}^{N-1}\left(y_{z}^{*}[n] \hat{A} e^{j(w n+\hat{\theta})}+y_{z}[n] \hat{A}^{*} e^{-j(w n+\hat{\theta})}\right. \\
& \left.\quad-\hat{A}^{*} e^{-j(w n+\hat{\theta})} \hat{A} e^{j(w n+\hat{\theta})}\right) \\
& >\ln \gamma
\end{aligned}
$$

To solve the likelihood function, the value for $\hat{A}$ which maximizes the log-likelihood function must be determined. First, equation 2.65 is presented in vector notation for ease of analysis:

$$
\ln L(\mathbf{y})=\frac{1}{\sigma_{z}^{2}}\left[\mathbf{y}^{H} \mathbf{e} \hat{A} e^{j \hat{\theta}}+\hat{A}^{H} e^{-j \hat{\theta}} \mathbf{e}^{H} \mathbf{y}-\hat{A}^{H} e^{-j \hat{\theta}} \mathbf{e}^{H} \mathbf{e} \hat{A} e^{j \hat{\theta}}\right]
$$


where $\mathbf{e}=\left[1 e^{j \omega} \ldots e^{j \omega(N-1)}\right]^{T}$ and $\mathbf{y}=\left[y_{z}[0] y_{z}[1] \ldots y_{z}[N-1]\right]^{T}$. The derivative is then [11]:

$$
\begin{aligned}
\frac{\partial \ln L(\mathbf{y})}{\partial \hat{A}} & =\frac{1}{\sigma_{z}^{2}}\left[\left(\mathbf{e}^{H} e^{-j \hat{\theta}} \mathbf{y}\right)^{*}+0-\left(\mathbf{e}^{H} \mathbf{e} \hat{A}\right)^{*}\right] \\
& =\frac{1}{\sigma_{z}^{2}}\left[\mathbf{e}^{H} e^{-j \hat{\theta}} \mathbf{y}-\mathbf{e}^{H} \mathbf{e} \hat{A}\right]^{*}
\end{aligned}
$$

Set the derivative to zero and solve for $\hat{A}$ :

$$
\begin{aligned}
0 & =\mathbf{e}^{H} e^{-j \hat{\theta}} \mathbf{y}-\mathbf{e}^{H} \mathbf{e} \hat{A} \\
\hat{A} & =\frac{\mathbf{e}^{H} \mathbf{y} e^{-j \hat{\theta}}}{\mathbf{e}^{H} \mathbf{e}} \\
& =\frac{1}{N} \sum_{n=0}^{N-1} y_{z}[n] e^{-j(\omega n+\hat{\theta})}
\end{aligned}
$$

Substituting this MLE of $\hat{A}$ into equation 2.65, the log-likelihood equation becomes:

$$
\begin{aligned}
\ln L(\mathbf{y})= & \left.\frac{1}{\sigma_{z}^{2}} \sum_{n=0}^{N-1} y_{z}^{*}[n]\left(\frac{1}{N} \sum_{n=0}^{N-1} y_{z}[n] e^{-j(\omega n+\hat{\theta})}\right) e^{j(w n+\hat{\theta})}\right) \\
& +\frac{1}{\sigma_{z}^{2}} \sum_{n=0}^{N-1}\left(\frac{1}{N} \sum_{n=0}^{N-1} y_{z}^{*}[n] e^{j(\omega n+\hat{\theta})}\right) e^{-j(w n+\hat{\theta})} y_{z}[n] \\
& -\frac{1}{\sigma_{z}^{2}} \sum_{n=0}^{N-1}\left[\left(\frac{1}{N} \sum_{n=0}^{N-1} y_{z}^{*}[n] e^{j(\omega n+\hat{\theta})}\right) e^{-j(w n+\hat{\theta})}\right. \\
\ln L(\mathbf{y})= & \left.\left.\frac{1}{\sigma_{z}^{2}}(1+1-1) \frac{1}{N} \mid \sum_{n=0}^{N-1} y_{z=0}^{N-1} y_{z}[n] e^{-j(\omega n+\hat{\theta})}\right) e^{j(w n+\hat{\theta})}\right] \\
\ln L(\mathbf{y})= & \frac{1}{\sigma_{z}^{2}} P[k]>\ln \gamma
\end{aligned}
$$


where $\mathrm{P}[\mathrm{k}]$ is the $\mathrm{k}^{\text {th }}$ frequency bin of the periodogram corresponding to $\omega=\frac{2 \pi k}{N}$. Therefore, for the case of a single sinusoid, with unknown amplitude and phase, the GLRT decides $\mathcal{H}_{1}$ for:

$$
T(\mathbf{y})=P[k]>\sigma_{z}^{2} \ln \gamma=\gamma^{\prime}
$$

where $\gamma^{\prime}$ is the new threshold under the test condition $\mathrm{T}(\mathbf{y})$.

$\mathrm{T}(\mathbf{y})$ is a chi-square-distributed random variable. Under condition $\mathcal{H}_{0}, \mathrm{~T}(\mathbf{y})$ is a central chi-squared distribution with two degrees of freedom and a probability density function given by [14]:

$$
p_{n}(u)=\frac{1}{\sigma_{z}^{2}} e^{-\frac{u}{\sigma_{z}^{2}}}
$$

Under condition $\mathcal{H}_{1}, \mathrm{~T}(\mathbf{y})$ is a noncentral chi-squared distribution with two degrees of freedom and a probability density function given by:

$$
p_{s}(u)=\frac{1}{\sigma_{z}^{2}} e^{-\frac{\left(\lambda^{2}+u\right)}{\sigma_{z}^{2}}} I_{0}\left(2 \sqrt{u} \frac{\lambda}{\sigma_{z}^{2}}\right)
$$

where $\lambda$ is the noncentrality parameter given by:

$$
\lambda=\sqrt{N} A
$$

The probability of false alarm is then given by:

$$
\begin{aligned}
P_{F A} & =p\left(P[k]>\gamma^{\prime} ; \mathcal{H}_{0}\right) \\
& =1-\int_{0}^{\gamma^{\prime}} p_{n}(u) d u
\end{aligned}
$$


while the probability of detection is given by:

$$
\begin{aligned}
P_{D} & =p\left(P[k]>\gamma^{\prime} ; \mathcal{H}_{1}\right) \\
& =1-\int_{0}^{\gamma^{\prime}} p_{s}(u) d u
\end{aligned}
$$

\section{The GLRT for Unknown Frequency, Amplitude and Phase}

The analysis in the previous section derived the periodogram as the realization of the GLRT for the case of a complex sinusoid with unknown amplitude and phase. For this case, the unknown parameter frequency was assumed known. This type of detector is referred to as a clairvoyant detector and its performance will be used as an upper bound on performance for the more general problem of unknown amplitude, frequency and phase.

For a single complex sinusoid, with unknown frequency, amplitude and phase, the GLRT decides $\mathcal{H}_{1}$ if:

$$
\max _{f} \frac{p\left(\mathbf{y} \mid \hat{A}, \hat{\theta}, f, \mathcal{H}_{1}\right)}{p\left(\mathbf{y} \mid \mathcal{H}_{0}\right)}>\ln \gamma
$$

for which the log-likelihood function is given by:

$$
\max _{f} \ln \frac{p\left(\mathbf{y} \mid \hat{A}, \hat{\theta}, f, \mathcal{H}_{1}\right)}{p\left(\mathbf{y} \mid \mathcal{H}_{0}\right)}>\ln \gamma
$$

Using the results from the previous section, equation 2.75 becomes:

$$
\max _{k} P[k]>\sigma_{z}^{2} \ln \gamma=\gamma^{\prime}
$$

Therefore, the GLRT detector for a sinusoid with unknown frequency, amplitude and phase is also the periodogram. The detector selects the largest peak produced by the 
periodogram and compares it to the threshold $\gamma^{\prime}$ to determine if a detection occurred.

The performance of this detector is similar to the previous case, except that all frequency bins are assessed. The resulting probability of false alarm and probability of detection for a single sinusoid with all parameters unknown are:

$$
P_{F A}=1-\left(\int_{0}^{\gamma^{\prime}} p_{n}(u) d u\right)^{N}
$$

and

$$
P_{D}=1-\left(\int_{0}^{\gamma^{\prime}} p_{n}(u) d u\right)^{N-1} \int_{0}^{\gamma^{\prime}} p_{s}(u) d u
$$

\section{Single Tone Detection for Averaged Periodograms}

Periodogram averaging is employed to reduce the variance of PSD estimates. The data is segmented into $\mathrm{K}$ blocks of length $\mathrm{L}$, the periodogram is calculated for each block and the results are averaged. The Bartlett averaged periodogram method is defined as [15]:

$$
P_{B}[k]=\frac{1}{K} \sum_{i=0}^{K-1} \frac{1}{L}\left|\sum_{n=0}^{L-1} y_{i}[n] e^{-j \omega n}\right|^{2}
$$

where,

$$
y_{i}[n]=y[i \cdot L+n]
$$

The Bartlett averaged periodogram is also a chi-square-distributed random variable. Under condition $\mathcal{H}_{0}, \mathrm{~T}(\mathbf{y})$ is a central chi-squared distribution with $2 \mathrm{~K}$ degrees of freedom and a probability density function given by:

$$
p_{n_{B}}(u)=\frac{u^{K-1} e^{-u\left(\frac{K}{\sigma_{z}^{2}}\right)}}{\left(\frac{\sigma_{z}^{2}}{K}\right)^{K}(K-1) !}
$$


Under condition $\mathcal{H}_{1}, \mathrm{~T}(\mathbf{y})$ is a noncentral chi-squared distribution with $2 \mathrm{~K}$ degrees of freedom and a probability density function given by:

$$
p_{s_{B}}(u)=\frac{u^{\frac{(K-1)}{2}}}{\left(\frac{\sigma_{z}^{2}}{K}\right)\left(\lambda_{B}\right)^{K-1}} e^{-\left(\lambda_{B}^{2}+u\right)\left(\frac{K}{\sigma_{z}^{2}}\right)} I_{K-1}\left(2 \sqrt{u} \lambda_{B}\left(\frac{K}{\sigma_{z}^{2}}\right)\right)
$$

where $\lambda_{B}$ is the non-centrality parameter given by:

$$
\lambda_{B}=\sqrt{L} A
$$

The probability of false alarm is then given by:

$$
P_{F A}=1-\left(\int_{0}^{\gamma^{\prime}} p_{n_{B}}(u) d u\right)^{L}
$$

and

$$
P_{D}=1-\left(\int_{0}^{\gamma^{\prime}} p_{n_{B}}(u) d u\right)^{L-1} \int_{0}^{\gamma^{\prime}} p_{s_{B}}(u) d u
$$

The Welch method uses overlapped and windowed periodograms for the generation of its spectral estimate [15]. The detection performance for the Welch method is much more difficult to analyze and its performance will be determined via Monte Carlo simulation.

\subsubsection{Detection of Multiple Sinusoids}

In Section 2.2.4 it was shown that under certain conditions, the ML estimator performance for multiple sinusoids approaches that of the single sinusoid case. The key conditions discussed were the requirement for appropriate filtering to reduce spectral leakage and a minimum signal separation of 4 frequency bins. 
The detection threshold of the GLRT detector is based on the MLE of the sinusoid parameters. Therefore, when the requirements for single sinusoid MLE performance is maintained, for the case of multiple sinusoids, the single sinusoid GLRT performance bounds can also be used.

At the beginning of this section, it was shown that signal detection is typically presented in the form of a ROC curve. Unfortunately, the ROC does not provide a useful performance indication for the multiple sinusoid case. The ROC curve is generated by plotting the $\mathrm{P}_{D}$ vs. $\mathrm{P}_{F A}$ and the problem lies in the definition of what a detection truly represents. From equation 2.78 and equation 2.76 , a detection is declared if the magnitude of the largest periodogram element exceeds the detection threshold. The detection does not differentiate between a detection caused by the signal component $\left(\mathrm{P}_{\text {DCorrect }}\right)$ and one caused by the noise components $\left(\mathrm{P}_{\text {DFalse }}\right)$. Aditionally, the detection will not differentiate between the multiple sinusoids. As a result, the detection performance for multiple sinusoids will be biased by the largest sinusoid present. Additionally, increasing the number of sinusoids will increase the probability of detection of one of the multiple sinusoids, but not necessarily the one of interest. The difference between $\mathrm{P}_{D}$ and $\mathrm{P}_{D C \text { orrect }}$ is presented in Figure 2.7.

To evaluate the detection performance of multiple sinusoids, the $\mathrm{P}_{D C o r r e c t}$ is used as a performance indicator. This technique only evaluates the detection performance at the FFT bins corresponding to the signals present. For the single sinusoid case, this corresponds to the case of a single sinusoid with known frequency and the probability of detection is given in equation 2.73. The multiple sinusoid performance can then be assessed by the degradation in performance of $\mathrm{P}_{D \text { Correct }}$ with respect to the single sinusoid case. When the $\mathrm{P}_{D C o r r e c t}$ curves for the single sinusoid and multiple sinusoid problems converge, the detection performance bounds for single sinusoid detection 


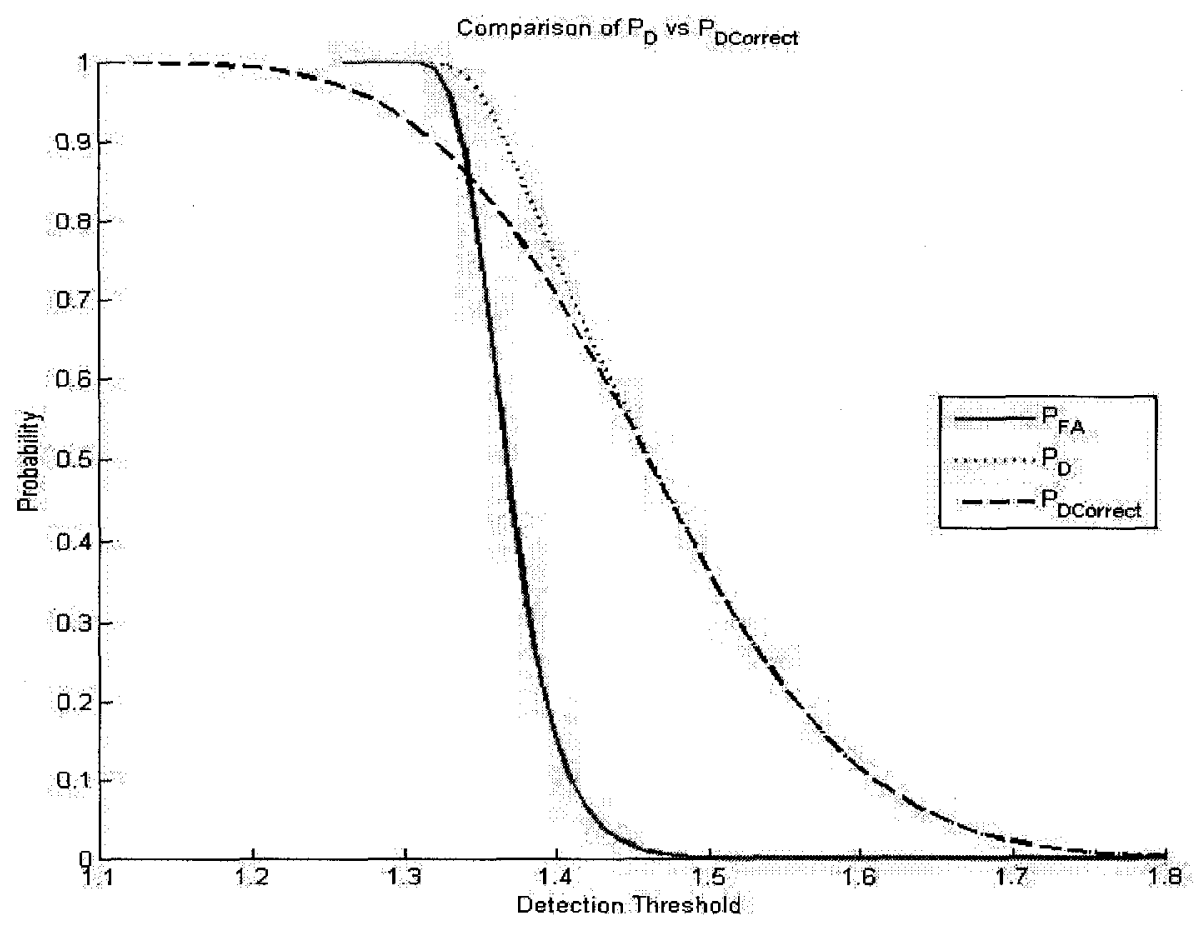

Figure 2.7: Detection performance comparison.

can be applied to the multiple sinusoid case.

\subsection{Bearing Estimation Performance Bounds}

In Section 2.2 it was discussed that, for an unbiased estimator, the CRLB provides a lower limit on the variance of the estimator for parameter $\alpha$.

The CRLB for bearing estimation has been developed by Stoica [16], for the case of a linear array. These results have been generalized for the case of a planar array [17]. The circular antenna array is a special case of the planar array and its bearing estimation performance is presented in this section.

The digital complex baseband signal vector for channel $\mathrm{x}$ is given by equation 2.29 and the vector of received data for all channels is given by: 


$$
\mathbf{y}_{B B}[n]=\left[\begin{array}{c}
\mathbf{y}_{B B_{1}}[n] \\
\mathbf{y}_{B B_{2}}[n] \\
\vdots \\
\mathbf{y}_{B B_{X}}[n]
\end{array}\right]=\left[\begin{array}{c}
\sum_{m=1}^{M} s_{B B_{1, m}}[n]+\eta_{B B_{1}}[n] \\
\sum_{m=1}^{M} s_{B B_{2, m}}[n]+\eta_{B B_{2}}[n] \\
\vdots \\
\sum_{m=1}^{M} s_{B B_{X, m}}[n]+\eta_{B B_{X}}[n]
\end{array}\right]
$$

which can be expressed as:

$$
\begin{aligned}
\mathbf{y}_{B B}[n]= & \mathbf{A}(\phi) \mathbf{s}_{B B}[n]+\eta_{B B}[n] \\
= & {\left[\begin{array}{cccc}
a_{1}\left(\phi_{1}\right) & a_{1}\left(\phi_{2}\right) & \ldots & a_{1}\left(\phi_{M}\right) \\
a_{2}\left(\phi_{1}\right) & a_{2}\left(\phi_{2}\right) & \ldots & a_{2}\left(\phi_{M}\right) \\
\vdots & \vdots & \ddots & \vdots \\
a_{X}\left(\phi_{1}\right) & a_{X}\left(\phi_{2}\right) & \ldots & a_{X}\left(\phi_{M}\right)
\end{array}\right]\left[\begin{array}{c}
s_{B B_{1}}[n] \\
s_{B B_{2}}[n] \\
\vdots \\
s_{B B_{M}}[n]
\end{array}\right]+\left[\begin{array}{c}
\eta_{B B_{1}}[n] \\
\eta_{B B_{2}}[n] \\
\vdots \\
\eta_{B B_{M}}[n]
\end{array}\right] }
\end{aligned}
$$

In Equation 2.84, $\mathbf{s}_{B B}[\mathrm{n}]$ represents the $\mathbf{M}$ sinusoids observed at the center of the array, while $\mathbf{A}(\phi)$ represents the complex array response or array manifold. The $m^{\text {th }}$ column of the array manifold, referred to as the Source Position Vector (SPV), indicates the array response due to a signal with bearing $\phi_{m}$. Bearing estimation is performed by searching the array manifold against all combinations of $\phi_{m}$ to estimate the true signal bearings. It is important to note that the array manifold is a function of the antenna array geometry; therefore, the positions of the antenna elements will impact the accuracy of the bearing estimates. The elements of the array manifold represented as a function of antenna position are given by:

$$
a_{x}\left(\phi_{m}\right)=e^{-j \pi\left(r_{x}(x) \cos \phi_{m}+r_{y}(x) \sin \phi_{m}\right)}
$$

where $\mathrm{r}_{x}(x)$ and $\mathrm{r}_{y}(x)$ are the positions of the antenna element $\mathrm{x}$ along the $\mathrm{x}$-axis and 
$y$-axis of the planar array, normalized to units of half wavelengths. Therefore, the normalized antenna radius is:

$$
r_{x}^{2}(x)+r_{y}^{2}(x)=\frac{2 R}{\lambda}
$$

which is the aperture of the antenna array.

\subsubsection{The CRLB for Bearing Estimation}

The CRLB expression for bearing estimation was first derived by Stoica [16], and further described by Van Trees [18]. The derived CRLB is presented as follows:

$$
\operatorname{var}(\phi) \geq \frac{\sigma^{2}}{2 N}\left(\operatorname{Re}\left\{\mathbf{H} \odot \mathbf{P}_{s s}^{T}\right\}\right)^{-1}
$$

where $\mathbf{H}$ is given by:

$$
\mathbf{H}=\mathrm{D}^{*} P_{A}^{\perp} \mathrm{D}
$$

$\mathbf{P}_{s s}$ is the source covariance matrix given by:

$$
\mathbf{P}_{s s}=E\left\{s_{B B}[n] s_{B B}^{*}[n]\right\}
$$

$\odot$ is the Hadamard matrix product and $\mathbf{D}^{*}$ is the conjugate transpose of matrix $\mathbf{D}$.

The derivative matrix $\mathbf{D}$, in equation 2.89, represents the derivative of $\mathbf{A}(\phi)$ with 
respect to $\phi$ given by:

$$
\mathbf{D}=\left[\begin{array}{cccc}
\frac{\partial a_{1}\left(\phi_{1}\right)}{\partial \phi_{1}} & \frac{\partial a_{1}\left(\phi_{2}\right)}{\partial \phi_{2}} & \ldots & \frac{\partial a_{1}\left(\phi_{M}\right)}{\partial \phi_{M}} \\
\frac{\partial a_{2}\left(\phi_{1}\right)}{\partial \phi_{1}} & \frac{\partial a_{2}\left(\phi_{2}\right)}{\partial \phi_{2}} & \ldots & \frac{\partial a_{2}\left(\phi_{M}\right)}{\partial \phi_{M}} \\
\vdots & \vdots & \ddots & \vdots \\
\frac{\partial a_{X}\left(\phi_{1}\right)}{\partial \phi_{1}} & \frac{\partial a_{X}\left(\phi_{2}\right)}{\partial \phi_{2}} & \ldots & \frac{\partial a_{X}\left(\phi_{M}\right)}{\partial \phi_{M}}
\end{array}\right]
$$

while $P_{A}^{\perp}$ represents the orthogonal projection matrix with respect to $\mathbf{A}(\phi)[18]$ (or equivalently, the projection matrix onto the noise subspace) given by:

$$
P_{A}^{\perp}=\left[\mathbf{I}-\mathbf{A}(\phi)\left[\mathbf{A}^{*}(\phi) \mathbf{A}(\phi)\right]^{-1} \mathbf{A}^{*}(\phi)\right]
$$

Therefore, $\mathbf{H}$ is the orthogonal projection of the $\mathbf{D}^{*} \mathbf{D}$ onto a subspace orthogonal to the subspace spanned by the SPVs of $\mathbf{A}(\phi)$. Additionally, the term $\mathbf{D}^{*} \mathbf{D}$ represents the squared Euclidean norm or magnitude square of $\mathbf{D}$ [18].

\subsubsection{CRLB for Bearing of a Single Sinusoid}

For the case of a single sinusoid, the matrix $\mathbf{A}(\phi)$ reduces to a single SPV given by:

$$
\mathbf{A}(\phi)=\left[\begin{array}{c}
a_{1}\left(\phi_{1}\right) \\
a_{2}\left(\phi_{1}\right) \\
\vdots \\
a_{X}\left(\phi_{1}\right)
\end{array}\right]
$$


the matrix $\mathbf{D}$ reduces to the column vector:

$$
\mathbf{D}=\left[\begin{array}{c}
\frac{\partial a_{1}\left(\phi_{1}\right)}{\partial \phi_{1}} \\
\frac{\partial a_{2}\left(\phi_{1}\right)}{\partial \phi_{1}} \\
\cdots \\
\frac{\partial a_{X}\left(\phi_{1}\right)}{\partial \phi_{1}}
\end{array}\right]
$$

and the matrix $\mathbf{P}_{s s}$ reduces to a the scalar constant $\mathbf{P}_{s s}$. Inserting these results into equation 2.88 produces the simplified CRLB given by:

$$
\begin{aligned}
\operatorname{var}(\phi) & \geq \frac{\sigma^{2}}{2 N P_{s s}} \frac{1}{\mathbf{D}^{*} P_{A}^{\perp} \mathbf{D}} \\
& =\frac{1}{2 N \mathrm{snr}} \mathrm{D}^{*} P_{A}^{\perp} \mathbf{D}
\end{aligned}
$$

To solve the CRLB, $\mathbf{A}(\phi)$ can be represented as a curve spanning an X-dimensional subspace [17] and applying differential geometry. An arbitrary $\mathbf{A}(\phi)$ curve is shown in Figure 2.8.

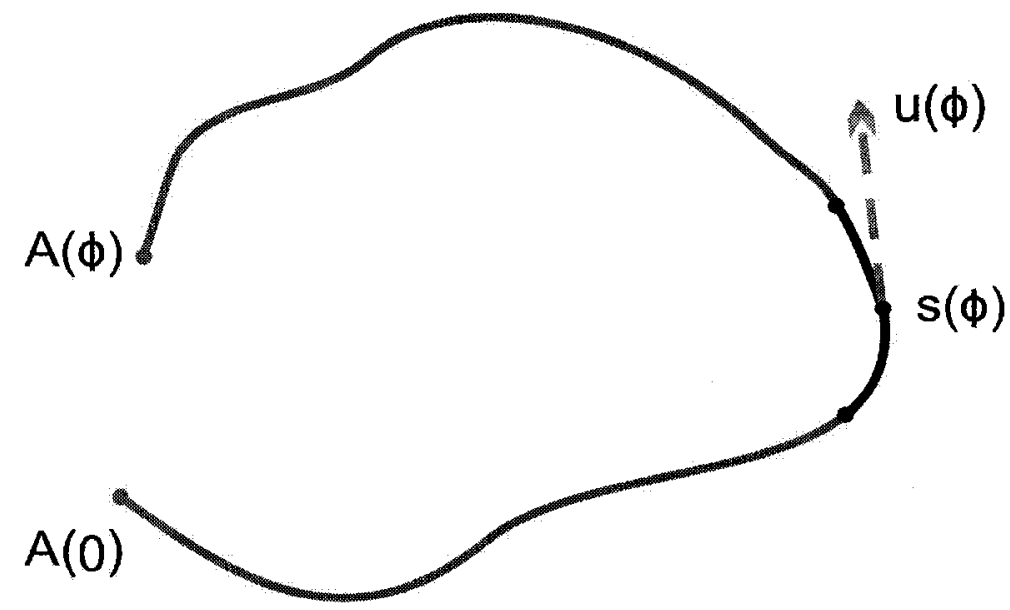

Figure 2.8: Example of a curve $\mathbf{A}(\phi)$, an arc on the curve $\mathbf{s}(\phi)$ and its unit tangent $\mathbf{u}(\phi)$. 
The arc $\mathbf{s}(\phi)$ in Figure 2.8 is of length $\mathbf{s}$. Of particular interest is the rate of change of $\mathbf{s}$ and unit tangent the vector $\mathbf{u}(\phi)$ given by:

$$
\frac{\partial \mathbf{s}(\phi)}{\partial \phi}=|\mathbf{D}|=\left[\begin{array}{c}
\pi\left(-r_{x}(1) \sin \phi_{1}+r_{y}(1) \cos \phi_{1}\right) \\
\pi\left(-r_{x}(2) \sin \phi_{1}+r_{y}(2) \cos \phi_{1}\right) \\
\vdots \\
\pi\left(-r_{x}(X) \sin \phi_{1}+r_{y}(X) \cos \phi_{1}\right)
\end{array}\right]
$$

and

$$
\mathbf{u}(\phi)=\frac{\partial \mathbf{A}(\phi)}{\partial \mathbf{s}(\phi)}=\frac{\mathbf{D}}{\frac{\partial \mathbf{s}(\phi)}{\partial \phi}}
$$

Rearranging the terms of 2.98 produces the relationship:

$$
\mathbf{D}=\frac{\partial \mathbf{s}(\phi)}{\partial \phi} \mathbf{u}(\phi)
$$

Substituting equation 2.99 into equation 2.95, the CRLB for bearing becomes:

$$
\operatorname{var}(\phi) \geq \frac{1}{2 N \operatorname{snr}\left[\frac{\partial \mathbf{s}^{*}(\phi)}{\partial \phi} \frac{\partial \mathbf{s}(\phi)}{\partial \phi}\right] \mathbf{u}^{*}(\phi) P_{A}^{\perp} \mathbf{u}(\phi)}
$$

By choosing the phase reference to be at the array centroid, $\mathbf{u}(\phi)$ can be made orthogonal to $\mathbf{A}(\phi)[17]$, therefore:

$$
P_{A}^{\perp} \mathbf{u}(\phi)=\mathbf{u}(\phi)
$$

and the CRLB for bearing becomes:

$$
\operatorname{var}(\phi) \geq \frac{1}{2 N \operatorname{snr}\left[\frac{\partial \mathbf{s}^{*}(\phi)}{\partial \phi} \frac{\partial \mathbf{s}(\phi)}{\partial \phi}\right] \mathbf{u}^{*}(\phi) \mathbf{u}(\phi)}
$$


The unit tangent vector has a magnitude of 1 , therefore:

$$
\operatorname{var}(\phi) \geq \frac{1}{2 N \operatorname{snr}\left[\frac{\partial \mathbf{s}^{*}(\phi)}{\partial \phi} \frac{\partial \mathbf{s}(\phi)}{\partial \phi}\right]}
$$

The circular array is a balanced symmetric array (i.e., the vectors $\mathbf{r}_{x}$ and $\mathbf{r}_{y}$ are orthogonal and have the same magnitude). Since the two vectors have the same magnitude, $\left|\mathbf{r}_{x}\right|=\left|\mathbf{r}_{y}\right|$, where || represents the Euclidean norm of the specified vector. Substituting this result into equation 2.97 produces:

$$
\frac{\partial \mathbf{s}^{*}(\phi)}{\partial \phi} \frac{\partial \mathbf{s}(\phi)}{\partial \phi}=\left(\pi\left|\mathbf{r}_{x}\right|\right)^{2}
$$

and the CRLB for bearing, for a circular antenna array is given by:

$$
\operatorname{var}(\phi) \geq \frac{1}{2 N \operatorname{snr} \pi^{2}\left|\mathbf{r}_{x}\right|^{2}}
$$

The expression $\left|\mathbf{r}_{x}\right|$ in equation 2.105 is the Euclidean norm of the vector $\mathbf{r}_{x}$, therefore:

$$
\left|\mathbf{r}_{x}\right|^{2}=\sum_{x=1}^{X} r_{x}^{2}(x)=\left(\frac{2 R}{\lambda}\right)^{2} \sum_{x=1}^{X}\left[\cos \left(\frac{2 \pi x}{X}\right)\right]^{2}
$$

From equations 2.105 and 2.106, it is observed that the CRLB for bearing is a function of wavelength. When calculating the CRLB for performance across a frequency band, the highest frequency component must be used, corresponding to a weaker bound, applicable to all frequencies of interest. 


\section{Multiple Sinusoid Bearing Estimation Performance}

The complexity of the CRLB for bearing estimation increases rapidly with the number of number of signals present[17]. While the single sinusoid performance bound is a function of SNR and antenna geometry, the multiple sinusoid problem is also influenced by the relative angular separation of the measured signals.

However, as discussed in Section 2.2.4, if appropriate windowing is employed and the relative frequency separation of the sinusoids exceeds four FFT bins, the accuracy of the multiple sinusoid parameter estimates approach those of the single sinusoid case. Under these conditions, the performance of the bearing estimates will be bounded by the CRLB presented in equation 2.105. 


\section{Chapter 3}

\section{Detection and Bearing Estimation Algorithms}

In the previous chapter, it was shown that FFT based estimators are approximate maximum likelihood estimators for the case of multiple, reasonably spaced sinusoids

( $\geq 4$ frequency bins) [8] in a low SNR environment. Furthermore, the performance bounds for each of the individual sinusoids approach that of the single sinusoid case when the sinusoids are reasonably spaced. Therefore, in this thesis, we will focus on FFT based detection and bearing estimation techniques.

The FFT based detection and bearing estimation algorithms investigated in this report are composed of three signal processing blocks: PSD Computation, Detection and Bearing Estimation. The signal processing elements are illustrated in Figure 3.1.

The two detection and estimation techniques compared are the FFT Cross-Correlation Eigen Decomposition technique and the FFT Interferometer Technique. The major differences between the algorithms are realized in the PSD and bearing estimation blocks of Figure 3.1. Additionally, the FFT processing may or may not include windowing; therefore, the performance of the two algorithms will be studied in conjunction with various windowing options. 


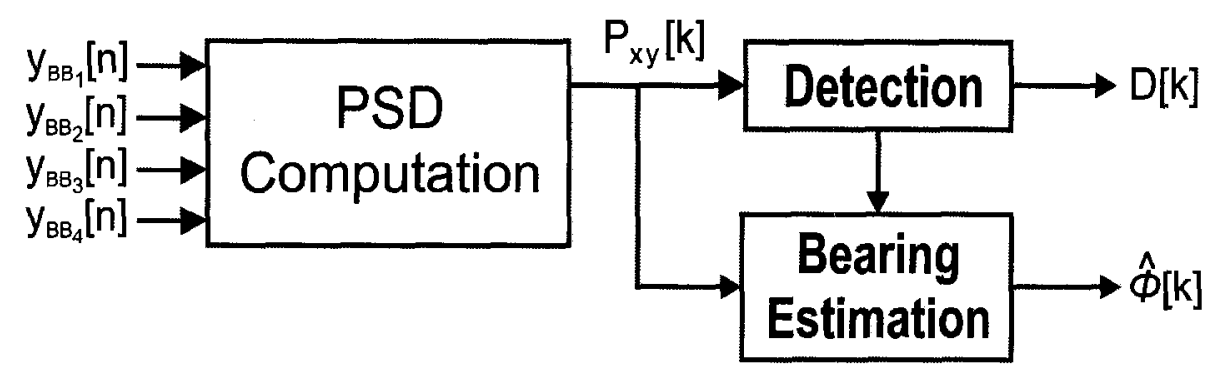

Figure 3.1: The signal processing model, where the subscripts $\mathrm{x}, \mathrm{y}$ represent the antenna numbers for an antenna pair.

For ease of analysis, it is assumed that $\omega_{m}=\frac{2 \pi k_{m}}{L}$, where $k_{m} \in(0, \mathrm{~L}-1)$ and an L-point FFT is performed. This implies that all tone frequencies correspond exactly to one of the FFT bins. For this special case, the fine estimation stage is not required. However, the simulations will contain experiments with off-bin frequencies.

\subsection{PSD Computation}

The PSD computation consists of four elements, FFT processing, cross-correlation and averaging, as illustrated in Figure 3.2.

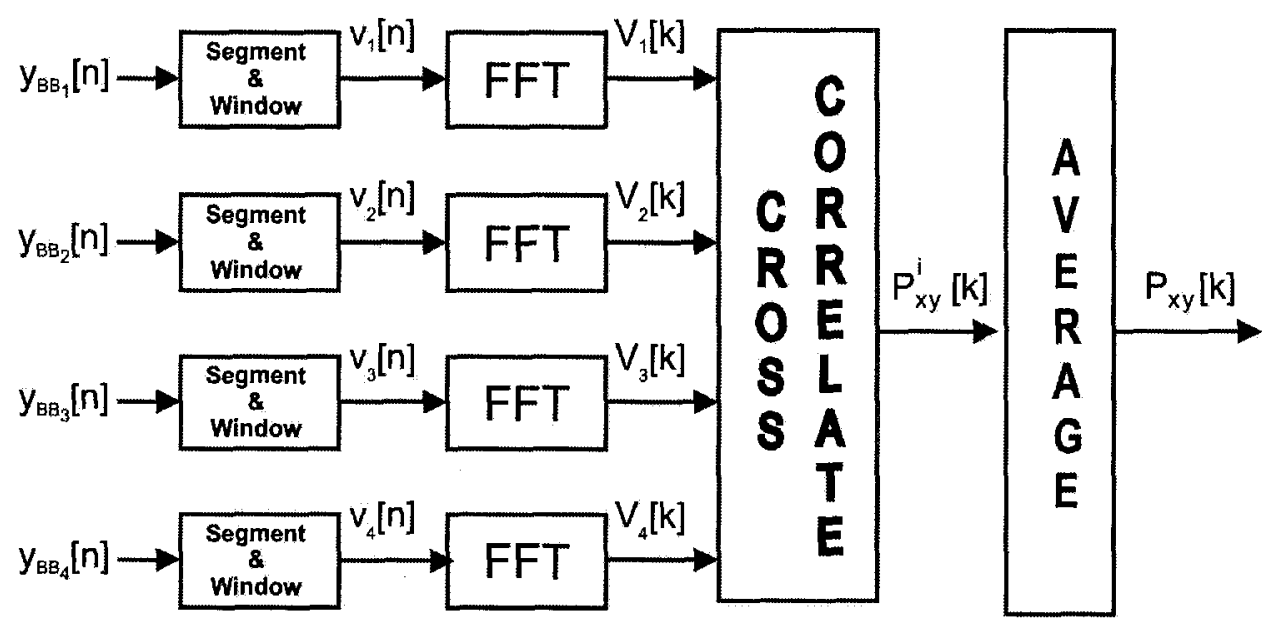

Figure 3.2: The processing elements of the PSD Computation Block. 


\subsubsection{Signal Segmentation and Windowing}

The antenna array receives $\mathrm{M}$ signals on each of the $\mathrm{X}$ antenna elements. The digital complex baseband signal captured on antenna $\mathrm{x}$, from equation 2.29 is:

$$
y_{B B_{x}}[n]=\sum_{m=1}^{M} A_{m} e^{j\left[\omega_{m} n+\theta_{x, m}\right]}+\eta_{B B_{x}}[n]
$$

A total of $\mathrm{N}$ samples are recorded for each detection and bearing estimation calculation. The $\mathrm{N}$ samples are segmented into $\mathrm{K}$ blocks of length $\mathrm{L}<\mathrm{N}$ to facilitate FFT averaging, which is required for reduction of the signal variance.

The Bartlett method segments the $\mathrm{N}$ samples of $\mathrm{y}_{x}[n]$ into $\mathrm{K}$ non-overlapping segments of length L. Additionally, the input samples are not windowed.

The Welch method segments the $\mathrm{N}$ samples of $\mathrm{y}_{x}[n]$ into K overlapping segments of length L, with each segment offset by D samples. Typically, a Hann window function is used to reduce the spectral leakage for each L-point segment.

\subsubsection{FFT Computation}

The second stage of signal processing is the computation of the FFT on the blocks

of input data. The FFT operation includes an optional window function w[n], used to reduce spectral leakage. The windowed data sequence for block $\mathrm{i}$ on channel $\mathrm{x}$ is given by:

$$
v_{x}^{i}[n]=\sum_{m=1}^{M} A_{m} w[n] e^{j\left[w_{m} n+\theta_{x, m}+\theta_{i}\right]}+\eta_{B B_{x}}[n] w[n]
$$

where $\theta_{i}$ is the phase offset of the signal due to the offset of the $\mathrm{i}^{\text {th }}$ block from the start of the original data sequence. The FFT of the windowed data sequence is given 
by:

$$
\begin{aligned}
V_{x}^{i}[k] & =\sum_{n=0}^{L-1} v_{x}^{i}[n] e^{-j \omega n} \\
& =\sum_{n=0}^{L-1} \sum_{m=1}^{M} A_{m} w[n] e^{j\left[\omega_{m} n+\theta_{x, m}+\theta_{i}\right]} e^{-j \omega n}+N_{x}[k] \\
& =\sum_{m=1}^{M} A_{m} e^{j\left[\theta_{x, m}+\theta_{i}\right]}\left[\sum_{n=0}^{L-1} w[n] e^{-j\left(\omega-\omega_{m}\right) n}\right]+N_{x}[k] \\
V_{x}^{i}[k] & =\sum_{m=1}^{M} A_{m} e^{j\left[\theta_{x, m}+\theta_{i}\right]} W\left[k-k_{m}\right]+N_{x}[k]
\end{aligned}
$$

where $W\left[k-k_{m}\right]$ is a circularly shifted version of $W[k]$. The spectrum of the input signal segment $\mathrm{Y}[\mathrm{k}]$, window spectrum $\mathrm{W}[\mathrm{k}]$ and windowed data sequence $\mathrm{V}[\mathrm{k}]$, for a sinusoid with $\omega_{m}=\frac{\pi}{2}$ and a 32 point Hann window is illustrated in Figure 3.3:

a)

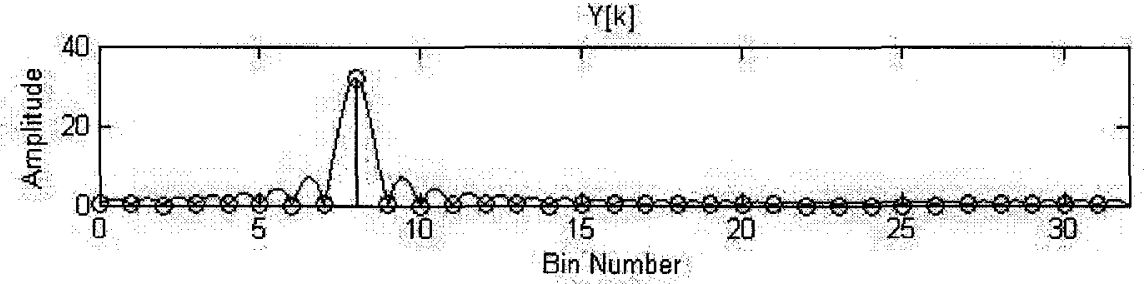

W[k]

b)

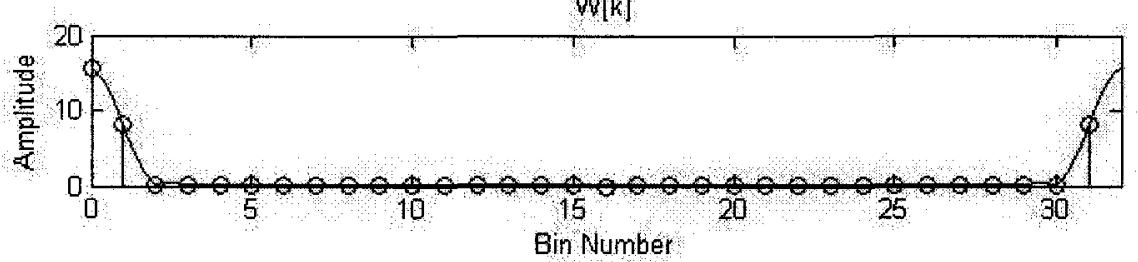

Yk]

c)

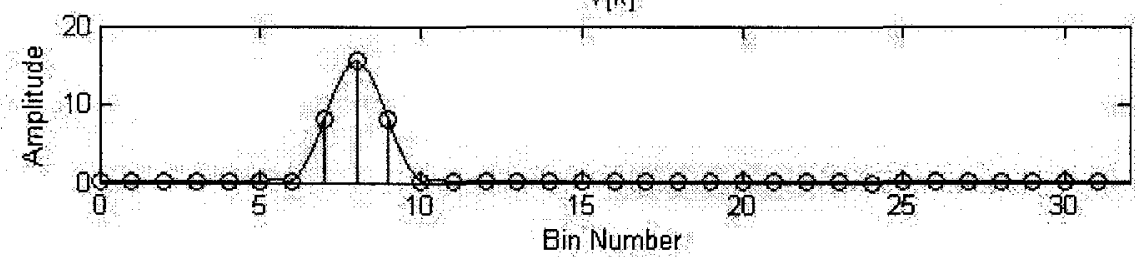

Figure 3.3: The solid line represents the DTFT spectrum, while the stem plot indicates the discrete DFT values. The three plots indicate the spectrum of the (a) input signal, (b) window function and (c) windowed input sequence. 
When the frequency of the sinusoid is known, the FFT can be designed to ensure the signal is centered on an FFT bin. For this case, the Bartlett method will provide superior performance, since it does not experience the processing loss associated with a window function. However, when the frequency of the sinusoid is unknown, the the worst case processing loss of the rectangular and Hann window functions must be considered.

\subsubsection{Cross-Correlation}

The third processing block of Figure 3.2 is cross-correlation, producing the multichannel PSD matrix [15], for a single realization of $\mathrm{v}[\mathrm{n}]=\left[\mathrm{v}_{1}[n] \mathrm{v}_{2}[n] \mathrm{v}_{3}[n] \mathrm{v}_{4}[n]\right]^{T}$.

The multichannel PSD matrix, illustrated in Figure 3.4, is Hermitian and positive semi-definite. The on-diagonal elements of the multichannel PSD matrix, highlighted in Figure 3.4 are the autospectral estimates, while the off-diagonal elements are the cross spectral estimates [15].

The power spectral density estimate for channel pair $\mathrm{x}, \mathrm{y}$ is given by:

$$
P_{x y}^{i}[k]=\frac{1}{L U} V_{x}^{i}[k] V_{y}^{i *}[k]
$$

The constant $U$ is a function of the selected window function and is required to remove the statistical bias produced by the window function. Without $U$, the power spectral density estimate will deviate from its true value. The term $U$ is given by:

$$
U=\frac{1}{L} \sum_{n=0}^{L-1}(w[n])^{2}
$$




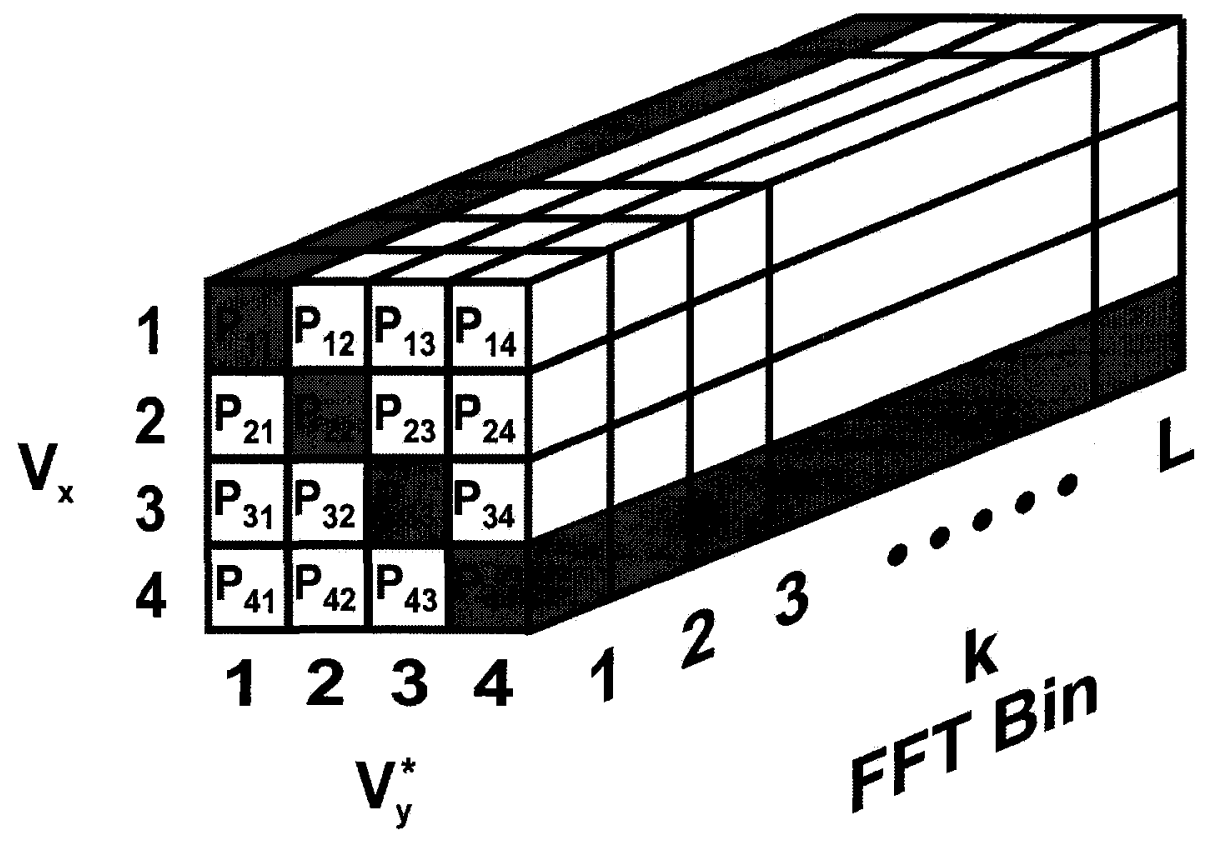

Figure 3.4: Multichannel Power Spectral Density matrix for v[n].

Substituting equation 3.2 into 3.3 the power spectrum estimate is given by:

$$
P_{x y}^{i}[k]=\frac{1}{L U}\left[\sum_{m=1}^{M} A_{m} e^{j\left[\theta_{x, m}+\theta_{i}\right]}+N_{x}[k]\right]\left[\sum_{m=1}^{M} A_{m} e^{-j\left[\theta_{y, m}+\theta_{i}\right]}+N_{y}^{*}[k]\right]
$$

As discussed in Section 2.2.4, it is desirable to implement FFT windowing and limit the minimum sinusoidal separation to four FFT bins. When these requirements are satisfied, single sinusoidal parameter estimation bounds may be applied to the multiple sinusoid problem. Under these conditions, a maximum of one sinusoid will fall in a particular FFT bin. Additionally, the window function will reduce the impact of the off bin sinusoids in equation 3.5, allowing them to be dropped. The power spectral estimate is then given by:

$$
P_{x y}^{i}[k]=\frac{1}{L U}\left[\left|A_{m}\right|^{2} e^{j \theta_{x-y, m}}\right]+\left[N_{x}[k] N_{y}^{*}[k]\right]
$$


where $\theta_{x-y, m}$ is the phase difference between the $\mathrm{m}^{\text {th }}$ sinusoid observed on channels x and $y$.

The PSD of the complex noise component of equation 3.6 is given by:

$$
P_{\eta}=\frac{N_{0} B}{L}
$$

The noise observed on the two channels is assumed to be uncorrelated, independently identically distributed AWGN.

\subsubsection{PSD Averaging}

The final processing block of Figure 3.2 is averaging of the multichannel PSD estimates. A total of $\mathrm{N}$ samples are recorded for detection and estimation. With FFT-based estimators, increasing $\mathrm{N}$ does not reduce the variance of the estimators. Instead, averaging of PSD estimates must be performed to reduce the variance. Several variations of PSD averaging exist for minimizing estimator variance. The two techniques studied in this report are the Bartlett Method and the Welch Method.

\section{The Bartlett Method}

The multichannel PSD estimate of segment $\mathrm{i}$ is given by equation 3.3 and the Bartlett

averaged multichannel PSD estimate, for $\mathrm{K}=\frac{N}{L}$ averaged segments, is given by:

$$
P_{x y}[k]=\frac{1}{K} \sum_{i=1}^{K} P_{x y}^{i}[k]
$$

With the Bartlett method, averaging $\mathrm{K}$ multichannel PSD estimates reduces the variance of the cross power spectral density estimates by a factor K. However, the cost of this variance reduction is a decrease in spectral resolution by a factor $\mathrm{K}$. 


\section{The Welch Method}

The multichannel PSD estimate of segment $i$ is given by equation 3.3 and the Welch averaged cross power spectral density estimate is also given by equation 3.8. However, the value for $\mathrm{K}$ is larger due to the overlapping of segments.

A special case of $50 \%$ overlap exists, which is computationally efficient and almost optimum. For the case of $50 \%$ overlap, $\mathrm{K}=\frac{2 N}{L}-1$. The Welch method has the same spectral resolution as the Bartlett method, with an additional estimator variance reduction [19] of $9 / 16$ or $2.5 \mathrm{~dB}$.

\subsection{Sinusoid Detection}

The averaged multichannel PSD estimate data $P_{x y}[k]$, is processed by the detection signal processing block to evaluate the presence of signals in the input data. The detection processing block uses the diagonal elements of $P_{x y}[k]$, which is the set of averaged periodograms for each of the $\mathrm{X}$ channels.

The output of the detection signal processing block is the detection vector $\mathbf{D}[\mathrm{k}]$, indicating the frequency bin of each detected signal.

\subsubsection{The Single Channel Periodogram Detector}

In many practical applications, a single channel is selected for the signal detection process, while the entire array is used for bearing estimation.

The detection performance for a single channel, using the Bartlett averaged periodogram was derived in Section 2.3.2. The PDF was determined to be a chi-square distribution with $2 \mathrm{~K}$ degrees of freedom. Under condition $\mathcal{H}_{0}$, the periodogram estimate is a central-chi square distribution and under condition $\mathcal{H}_{1}$, the periodogram 
estimate is a noncentral chi-square distribution with non-centrality parameter $\lambda_{B}=$ $\sqrt{L} A$.

The probability of false alarm and probability of detection are given be Equation 2.80 and Equation 2.81, respectively.

The performance for a single channel, using the Welch averaged periodogram is difficult to derive and its performance will be established via Monte-Carol simulations.

\subsubsection{The Multichannel Averaged Periodogram Detector}

By using the entire trace of the multichannel PSD matrix, increased detection performance is achieved, at the expense of a slight increase in computational complexity.

The averaged periodograms from the each of the $\mathrm{X}$ channels are averaged, producing the multichannel averaged periodogram. The multichannel averaged periodogram is given by:

$$
P_{\text {Multi }}[k]=\frac{1}{X} \sum_{x=1}^{X} P_{x x}[k]=\frac{1}{K X} \sum_{x=1}^{X} \sum_{i=1}^{K} P_{x x}^{i}[k]
$$

For the case of Bartlett averaged periodograms, the periodogram estimates have a chi-squared distribution, with $2 \mathrm{KX}$ degrees of freedom. Under condition $\mathcal{H}_{0}$, the periodogram estimate is a central-chi square distribution given by:

$$
p_{n_{B}}(u)=\frac{u^{K X-1} e^{-u\left(\frac{K X}{\sigma_{z}^{2}}\right)}}{\left(\frac{\sigma_{z}^{2}}{K X}\right)^{K X}(K X-1) !}
$$

Under condition $\mathcal{H}_{1}$, the periodogram estimate is a noncentral chi-square distribution given by:

$$
p_{s_{B}}(u)=\frac{u^{\frac{(K X-1)}{2}}}{\left(\frac{\sigma_{z}^{2}}{K X}\right)\left(\lambda_{B}\right)^{K X-1}} e^{-\left(\lambda_{B}^{2}+u\right)\left(\frac{K X}{\sigma_{z}^{2}}\right)} I_{K X-1}\left(2 \sqrt{u} \lambda_{B}\left(\frac{K X}{\sigma_{z}^{2}}\right)\right)
$$


with non-centrality parameter $\lambda_{B}=\sqrt{L} A$.

As with the single channel case, the detection performance for the Welch averaged periodogram will be determined via Monte-Carlo simulations.

\subsection{Bearing Estimation}

In the previous section, the trace of the multichannel PSD matrix was used to detect the presence of sinusoids. In this section, the off-diagonal elements of the multichannel PSD matrix will be used to calculate an AOA estimate for each of the detected signals.

Two methods of bearing estimation are studied in this thesis, the four channel interferometer and eigen-decomposition.

The four channel interferometer is a computationally efficient bearing estimation technique, which uses a subset of the multichannel PSD matrix. On the other hand, the eigen-decomposition technique is a computationally intensive method which utilizes the entire multichannel PSD matrix to generate bearing estimates.

For the case of a four channel eigen-decomposition, a closed form expression exists for the characteristic equation, whose roots are the eigenvalues, as well as a closed form solution for the roots. By exploiting the closed form solutions, a significant reduction in computational complexity can be realized.

Another eigen-decomposition technique, based on the power method is also studied. The power method is a less computationally demanding technique, at the cost of reduced bearing accuracy. 


\subsubsection{The Interferometer Technique}

The interferometer is a phase difference (or equivalently a time difference) technique for bearing estimation [20]. For the case of the four channel receiver, depicted in Figure 2.1, a four channel interferometer is utilized.

The four channel interferometer uses the phase difference between antenna pairs $[1,3]$ and $[2,4]$ to generate a bearing estimate $\hat{\phi}$. Using equation 2.4, the phase difference between the antenna 1 and antenna 3 is given by:

$$
\begin{aligned}
\theta_{13} & =\theta_{1}-\theta_{3} \\
& =\left(\frac{\Omega R}{c}\right)[\cos (\phi)-\cos (\phi-\pi)] \\
& =\left(\frac{2 \pi R}{\lambda}\right)[2 \cos (\phi)] \\
& =\frac{4 \pi R}{\lambda} \cos (\phi)
\end{aligned}
$$

Similarly,

$$
\begin{aligned}
\theta_{24} & =\theta_{2}-\theta_{4} \\
& =\left(\frac{\Omega R}{c}\right)\left[\cos \left(\phi-\frac{\pi}{2}\right)-\cos \left(\phi-\frac{3 \pi}{2}\right)\right] \\
& =\left(\frac{2 \pi R}{\lambda}\right)[\sin (\phi)-\sin (\phi-\pi)] \\
& =\frac{4 \pi R}{\lambda} \sin (\phi)
\end{aligned}
$$

The four channel interferometer uses equations 3.12 and 3.13 to determine the AOA 
of the target signal using:

$$
\begin{aligned}
\phi & =\tan ^{-1}\left(\frac{\sin (\phi)}{\cos (\phi)}\right) \\
& =\tan ^{-1}\left(\frac{\frac{4 \pi R}{\lambda} \sin (\phi)}{\frac{4 \pi R}{\lambda} \cos (\phi)}\right) \\
& =\tan ^{-1}\left(\frac{\theta_{24}}{\theta_{13}}\right)
\end{aligned}
$$

In Section 2.2.3, it was shown that the MLE of phase at each antenna is given by $\arg \left\{Z\left(e^{j \hat{\omega}}\right)\right\}$. Furthermore, the estimated phase difference between antenna $\mathrm{x}$ and $\mathrm{y}$, at frequency bin $\mathrm{k}$, is given by:

$$
\hat{\theta}_{x y}[k]=\arg \left\{P_{x y}[k]\right\}
$$

where $\mathrm{P}_{x y}[k]$ is the averaged PSD estimate given by equation 3.8. Substituting the phase difference estimates into equation 3.14, the AOA estimate for the sinusoid at frequency bin $\mathrm{k}$ is given by:

$$
\begin{aligned}
\hat{\phi}[k] & =\tan ^{-1}\left(\frac{\hat{\theta}_{24}[k]}{\hat{\theta}_{13}[k]}\right) \\
& =\tan ^{-1}\left(\frac{\arg \left\{P_{24}[k]\right\}}{\arg \left\{P_{13}[k]\right\}}\right)
\end{aligned}
$$

Unfortunately, by expressing the phase relationship as a ratio in equation 3.16 , the sign terms are disassociated from their respective phase terms, resulting in ambiguity between quadrants as depicted in Figure 3.5.

The phase ambiguity is corrected by incorporating the sign of $\arg \left\{P_{13}[k]\right\}$ and $\arg \left\{P_{24}[k]\right\}$ in the bearing estimation calculation. The bearing estimation is now given by: 


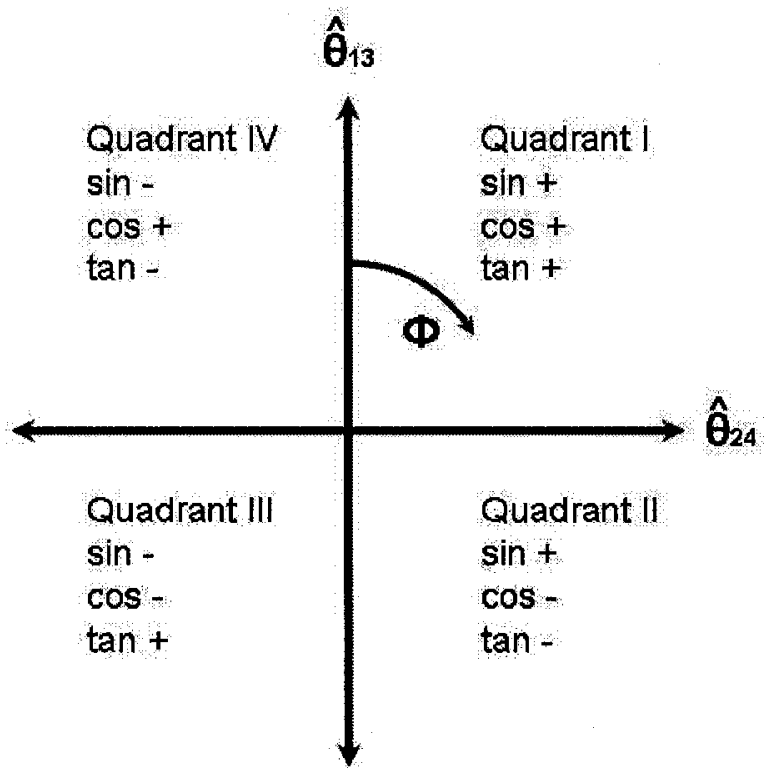

Figure 3.5: Ambiguity of $\tan ^{-1}$ due to the combined sign term.

$$
\hat{\phi}[k]= \begin{cases}\tan ^{-1}\left(\frac{\arg \left\{P_{24}[k]\right\}}{\arg \left\{P_{13}[k]\right\}}\right)+\pi & : \arg \left\{P_{13}[k]\right\}<0, \arg \left\{P_{24}[k]\right\}>0 \\ \tan ^{-1}\left(\frac{\arg \left\{P_{24}[k]\right\}}{\arg \left\{P_{13}[k]\right\}}\right)-\pi & : \arg \left\{P_{13}[k]\right\}<0, \arg \left\{P_{24}[k]\right\}<0 \\ \tan ^{-1}\left(\frac{\arg \left\{P_{24}[k]\right\}}{\arg \left\{P_{13}[k]\right\}}\right) & : \text { otherwise }\end{cases}
$$

For each bearing estimate, only the $\mathrm{P}_{13}$ and $\mathrm{P}_{24}$ components, at frequency bin $\mathrm{k}$ of the multichannel PSD matrix are required. The utilization of the multichannel PSD matrix, for the four channel interferometer, is shown in Figure 3.6.

As such, only a portion of the multichannel PSD matrix needs to be computed. The relatively low computational complexity of the interferometer makes it a favorable AOA technique for smaller, less capable signal processing platforms. 


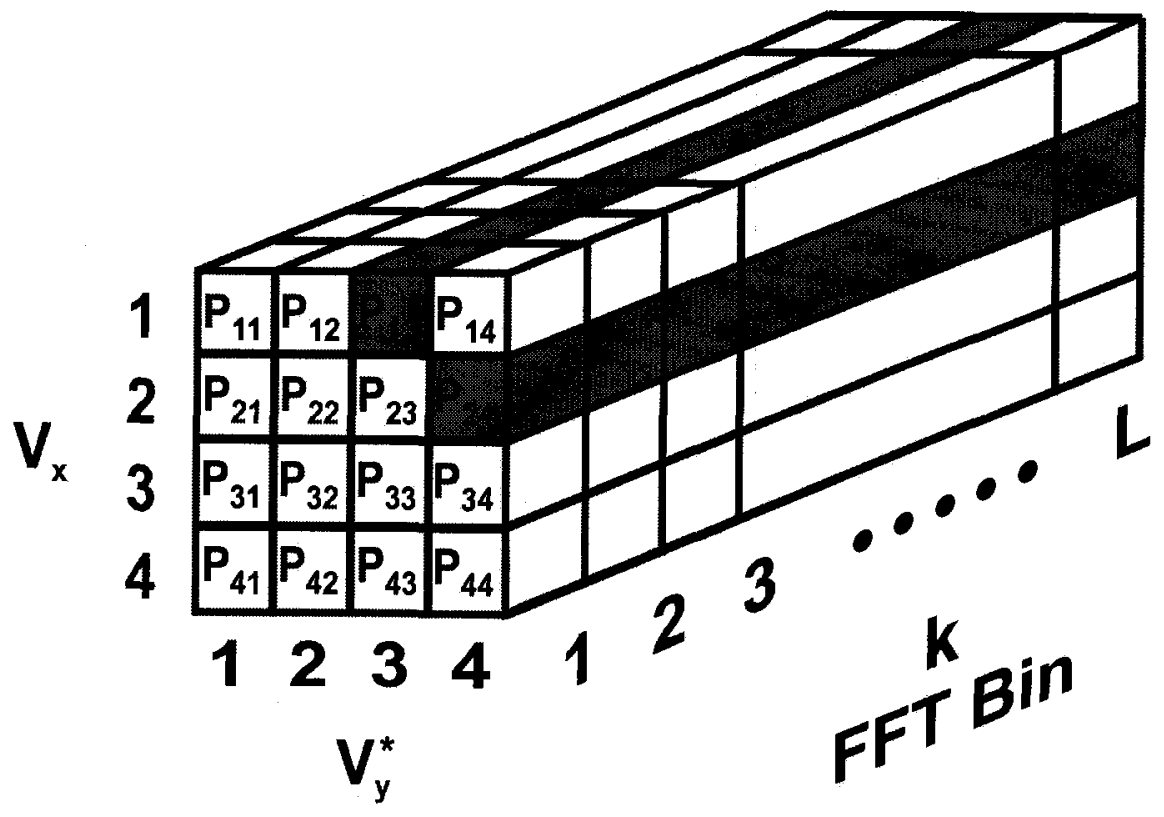

Figure 3.6: Multichannel PSD matrix utilization for four channel Interferometer.

\subsubsection{The Eigen-Decomposition Technique}

Eigen-decomposition is a diagonalization technique, which decomposes the matrix $\mathbf{P}$ into the product of its eigenvalues and eigenvectors as follows [18] [21] [22]:

$$
\mathbf{P} \Delta=\Delta \Lambda
$$

Applying the eigen-decomposition technique to the bearing estimation problem, equation 3.18 becomes:

$$
\mathbf{P}[k] \boldsymbol{\Delta}=\boldsymbol{\Delta} \boldsymbol{\Lambda}
$$

where $\mathbf{P}[\mathrm{k}]$ is the slice of the multichannel PSD matrix corresponding to frequency 
bin $\mathrm{k}$, given by:

$$
\mathbf{P}[k]=\left[\begin{array}{cccc}
P_{11}[k] & P_{12}[k] & P_{13}[k] & P_{14}[k] \\
P_{21}[k] & P_{22}[k] & P_{23}[k] & P_{24}[k] \\
P_{31}[k] & P_{32}[k] & P_{33}[k] & P_{34}[k] \\
P_{41}[k] & P_{42}[k] & P_{43}[k] & P_{44}[k]
\end{array}\right]
$$

$\Delta$ is the array of eigenvectors, given by:

$$
\Delta=\left[\delta_{1} \delta_{2} \delta_{3} \delta_{4}\right]=\left[\begin{array}{llll}
\delta_{11} & \delta_{21} & \delta_{31} & \delta_{41} \\
\delta_{12} & \delta_{22} & \delta_{32} & \delta_{42} \\
\delta_{13} & \delta_{23} & \delta_{33} & \delta_{43} \\
\delta_{14} & \delta_{24} & \delta_{34} & \delta_{44}
\end{array}\right]
$$

and $\Lambda$ is the diagonal matrix of eigenvalues, given by:

$$
\Lambda=\left[\begin{array}{cccc}
\lambda_{1} & 0 & 0 & 0 \\
0 & \lambda_{2} & 0 & 0 \\
0 & 0 & \lambda_{3} & 0 \\
0 & 0 & 0 & \lambda_{4}
\end{array}\right]
$$

Inserting equations 3.21 and 3.22 into equation 3.19 yields the fundamental eigenequation:

$$
\left(\mathbf{P}[k]-\lambda_{i} \mathbf{I}\right) \delta_{i}=\mathbf{0}
$$

Eigen-decomposition based bearing estimation is a three step process. The first step is the computation of the eigenvalues $\left(\lambda_{i}\right)$ of matrix $\mathbf{P}[\mathrm{k}]$. The second step uses the computed eigenvalues to calculate the corresponding eigenvectors. The third and final step is computation of a bearing estimate from the computed eigenvectors. 


\section{Computation of the Eigenvalues}

The eigenvalues of matrix $\mathbf{P}[\mathrm{k}]$ are computed in two stages. First, the characteristic polynomial for matrix $\mathbf{P}[\mathrm{k}]$ is computed, then the eigenvalues are calculated from the characteristic polynomial.

An examination of equation 3.23 reveals that for a non-trivial eigenvector solution:

$$
\operatorname{rank}\left(\mathbf{P}[k]-\lambda_{i} \mathbf{I}\right)<\operatorname{dim}\left(\mathbf{P}[k]-\lambda_{i} \mathbf{I}\right)=X
$$

This implies that:

$$
\operatorname{det}\left(\mathbf{P}[k]-\lambda_{i} \mathbf{I}\right)=\mathbf{0}
$$

which produces the characteristic equation [22].

Solving equation 3.25, with $\lambda=\lambda_{i}$ produces the characteristic polynomial of the form:

$$
\lambda^{X}+c_{1} \lambda^{X-1}+\ldots+c_{X-1} \lambda+c_{X}=0
$$

The derivation of the characteristic polynomial and its coefficients, for the case of a $4 \times 4$ matrix, is presented in Annex C.

Once the coefficients of the characteristic polynomial are calculated, the roots of the characteristic polynomial must be calculated to determine the eigenvalues. For the case of a $4 \times 4$ matrix, the characteristic polynomial is of degree 4 . Therefore, calculating the eigenvalues requires solving the quartic equation. A closed form solution to the quartic equation exists and an algorithm for performing this operation is presented in Annex D.

The eigenvalues for Hermitian matrices are real. Additionally, the magnitude of the eigenvalue indicates the relative strength of the corresponding signal. Therefore, 
the eigenvalues are ordered from largest to smallest in the eigenvalue array.

$$
\lambda_{1} \geq \lambda_{2} \geq \ldots \geq \lambda_{X}, \quad \lambda_{i} \in \lambda
$$

\section{Computation of the Eigenvectors}

Each eigenvector is computed separately by substituting its corresponding eigenvalue into equation 3.23 and solving the system of linear equations. The system of linear equations, for $\lambda_{1}$, and a $4 \times 4$ matrix is given by:

$$
\begin{aligned}
& \left(P_{11}[k]-\lambda_{1}\right) \delta_{11}+P_{12}[k] \delta_{12}+P_{13}[k] \delta_{13}+P_{11}[k] \delta_{14}=0 \\
& P_{21}[k] \delta_{11}+\left(P_{22}[k]-\lambda_{1}\right) \delta_{12}+P_{23}[k] \delta_{13}+P_{24}[k] \delta_{14}=0 \\
& P_{31}[k] \delta_{11}+P_{32}[k] \delta_{12}+\left(P_{33}[k]-\lambda_{1}\right) \delta_{13}+P_{34}[k] \delta_{14}=0 \\
& P_{41}[k] \delta_{11}+P_{42}[k] \delta_{12}+P_{43}[k] \delta_{13}+\left(P_{44}[k]-\lambda_{4}\right) \delta_{14}=0
\end{aligned}
$$

Several methods can be used to solve the linear system of equations, such as LU Decomposition and Gauss-Jordan Elimination [23]. The technique implemented in this thesis is Gauss-Jordan Elimination with pivoting. The pivoting option allows row swapping in the elimination process, avoiding round off and zero term errors.

\section{Computation of the Bearing Estimates}

The Eigen-Decomposition technique computes a bearing estimate by minimizing the cost function $\mathbf{C}(\phi)$, representing the mean square error associated with the error between the array manifold $\mathbf{A}(\phi)$ and the slice of the multichannel PSD matrix $\mathbf{P}[\mathrm{k}]$.

The cost $\mathbf{C}(\phi)$, associated with $\mathbf{A}(\hat{\phi})$ and the input signal $\mathbf{V}[\mathrm{k}]$ is the mean squared error between the two components. The error is given by the projection of $\mathrm{V}[\mathrm{k}]$ onto 
the subspace orthogonal to $\mathbf{A}(\hat{\phi})$, given by [18]:

$$
\mathbf{C}(\hat{\phi})=\operatorname{trace}\left\{\left|P_{A}^{\perp} \mathbf{V}[k]\right|^{2}\right\}
$$

where $P_{A}^{\perp}$ is the orthogonal projection matrix defined in equation 2.92 .

Expanding the magnitude squared operation produces:

$$
\mathbf{C}(\hat{\phi})=\operatorname{trace}\left\{P_{A}^{\perp} \mathbf{V}[k] \quad \mathbf{V}^{*}[k] P_{A}^{\perp}\right\}
$$

From equation 3.3, it is observed that $\mathbf{V}[\mathrm{k}] \mathbf{V}^{*}[\mathrm{k}]$ is a scaled version of $\mathbf{P}[\mathrm{k}]$. Therefore, the cost function can be interpreted as:

$$
\mathbf{C}(\hat{\phi})=\operatorname{trace}\left\{P_{A}^{\perp} \quad \mathbf{P}[k] \quad P_{A}^{\perp}\right\}
$$

The multichannel PSD matrix can be decomposed via eigenvector expansion as follows:

$$
\mathbf{P}[k]=\sum_{i=1}^{X} \lambda_{i} \delta_{i} \delta_{i}^{H}
$$

where the eigenvalues and eigenvectors have been computed in the previous section.

Substituting equation 3.35 into equation 3.34 , the cost function becomes:

$$
\mathbf{C}(\hat{\phi})=\sum_{i=1}^{X} \lambda_{i} \delta_{i}^{H} P_{A}^{\perp} P_{A}^{\perp} \delta_{i}
$$

As discussed in Section 2.2.4, when appropriate filtering is employed and the relative frequency separation is 4 bins or greater, the multiple tone parameter estimation performance approaches that of the single tone case. Under these conditions, the principle eigenvector can be attributed to the detected signal and the remain- 
ing eigenvectors correspond to the noise space. Therefore, the cost function can be optimized for the principle eigenvector and the remaining eigenvectors can be ignored. Reducing the cost function to consider a single signal and dropping the scalar eigenvalue produces:

$$
\mathbf{C}(\hat{\phi})=\delta_{1}^{H} P_{A}^{\perp} P_{A}^{\perp} \delta_{1}
$$

For the case of a four-element circular antenna array, discussed in Section 2.1, the SPV is given by:

$$
\mathbf{A}(\phi)=\left[\begin{array}{c}
a_{1}(\hat{\phi}) \\
a_{2}(\hat{\phi}) \\
a_{3}(\hat{\phi}) \\
a_{4}(\hat{\phi})
\end{array}\right]=\left[\begin{array}{c}
e^{j \frac{2 \pi R}{\lambda} \cos (\hat{\phi})} \\
e^{j \frac{2 \pi R}{\lambda} \cos \left(\hat{\phi}-\frac{\pi}{2}\right)} \\
e^{j \frac{2 \pi R}{\lambda} \cos (\hat{\phi}-\pi)} \\
e^{j \frac{2 \pi R}{\lambda} \cos \left(\hat{\phi}-\frac{3 \pi}{2}\right)}
\end{array}\right]
$$

Inserting 3.38 into equation 3.37 , the cost function for the four-element circular antenna array is given by:

$$
\mathbf{C}(\hat{\phi})=\frac{3}{4} \sum_{i=1}^{4}\left|\delta_{1 i}\right|^{2}-\frac{1}{4} \sum_{i=1}^{4} \sum_{\substack{j=1 \\ j \neq i}}^{4} \delta_{1 i} \delta_{1 j}^{*} e^{j \frac{2 \pi R}{\lambda}\left[\cos \left(\hat{\phi}-(i-1) \frac{\pi}{2}\right)-\cos \left(\hat{\phi}-(j-1) \frac{\pi}{2}\right)\right]}
$$

The bearing estimate $\hat{\phi}$ is acquired by searching the values of $\phi$ for the value which minimizes the cost function $\mathbf{C}(\hat{\phi})$. Several optimization techniques exist for locating minimum of a function. The steepest descent algorithm is a common technique which utilizes the gradient (first derivative of the function) to locate a local minimum [23]. The algorithm iterates through successive bearing estimates until the gradient equals zero, indicating a local minimum has been obtained. The successive bearing estimates, using the steepest descent algorithm are given by:

$$
\phi_{t+1}=\phi_{t}+\epsilon \frac{\partial \mathbf{C}\left(\hat{\phi}_{t}\right)}{\partial \phi}
$$


where $\epsilon$ is a small number greater than 0 , representing the algorithm's step size. An example cost function and its gradient are shown in Figure 3.7.

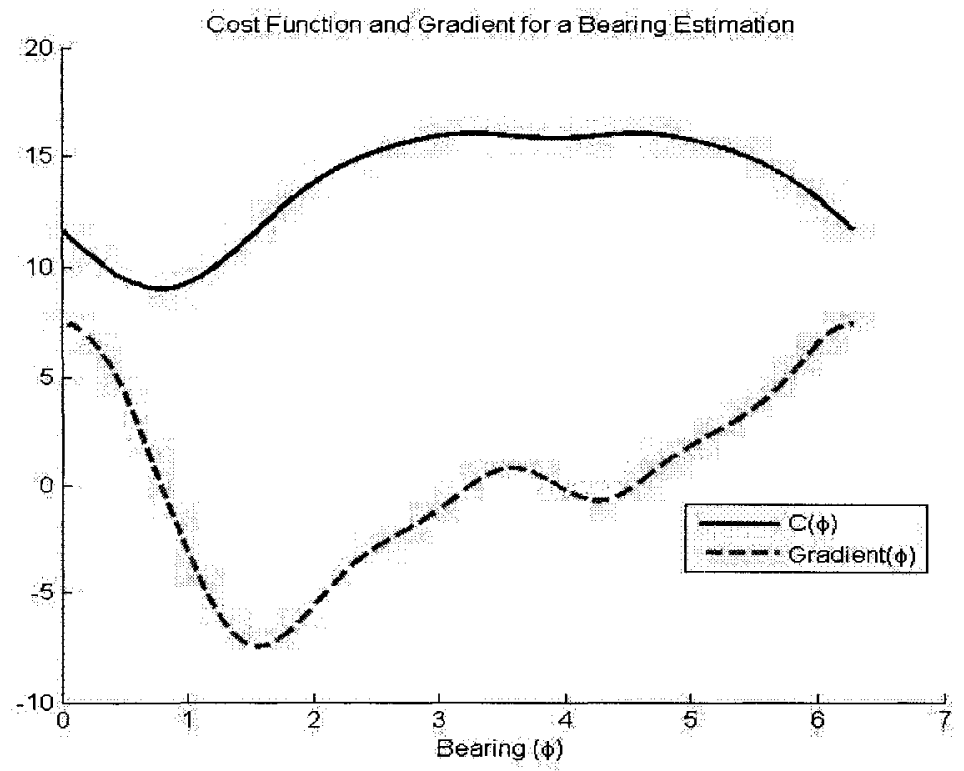

Figure 3.7: Cost function and gradient for a signal with bearing pi/4.

As illustrated in Figure 3.7, the cost function can have multiple local minimums. Care must be taken in selecting the initial bearing estimate to ensure the steepest descent algorithm finds the global minimum of the cost function. In this thesis, the initial bearing estimate is obtained by performing an interferometer bearing estimation and using the result as the starting point for the steepest descent algorithm.

\subsubsection{Eigen-Decomposition Technique using the Power Method}

The power method, also referred to as the power iteration method, provides an approximation of the primary eigenvalue of a square matrix [21] [22]. For large matrices the eigen-decomposition is difficult to solve and computationally demanding. For such matrices, the power method provides an efficient approximation of the primary 
eigenvalue. Due to the channelization performed by the FFT, only the primary eigenvalue is of interest, making the power method an attractive technique. However, since the power method is an iterative approximation technique, it will have greater error in comparison with the numerical eigen-decomposition.

In this thesis, the power method was considered. However, the square $(4 \times 4)$ matrix is small and the numerical eigen-decomposition was not computationally restrictive. For this case, the power method would not provide a significant reduction in computational complexity. Additionally, similar bearing estimation performance was observed for the interferometer and eigen-decomposition in Section 4.2. This also makes the power method less attractive since it produces less accurate bearing estimates in comparison with the numerical eigen-decomposition. Therefore, the performance of the power method was not further studied in this report. However, for antenna arrays with a greater number of elements, the size of the square matrix will also increase and the power method may become a more attractive approach. 


\section{Chapter 4}

\section{Results}

The results presented in this thesis were performed using MATLAB.

For each trial, an input signal was generated, based on the given simulation parameters (number of signals, SNR, frequency, phase and bearing). Each input signal consisted of one second of baseband data, sampled at a rate of $5 \mathrm{MHz}$ complex. The input signal is generated at baseband and represents the tuned signal with a $5 \mathrm{MHz}$ bandwidth and a carrier frequency of $57.5 \mathrm{MHz}$.

For all trials, the noise floor was created with a variance of $1\left(\sigma_{z}^{2}=1\right)$. The amplitude of each signal was calculated for the specified SNR, with reference to the noise floor. For the case of multiple sinusoids, each sinusoid was generated individually and summed at the particular antenna input.

The antenna configuration simulated in this thesis is a four channel circular array with a radius of 1 meter. Each channel is sampled independently and sampling is assumed to be phase coherent.

The goal of this thesis is to develop theoretical sinusoid detection and bearing estimation performance bounds and evaluate these bounds through Monte Carlo simulations. However, the next phase of this development is to implement the signal 
processing architecture on a real-time signal processing platform. Therefore, it is desirable to select simulation parameters which are representative of the intended hardware platform. Due to the weak signal strength of the sinusoids ( $\geq-45 \mathrm{~dB}$ ), a large FFT must be employed and an Field Programmable Gate Array (FPGA) is a favorable platform for supporting this requirement. The Virtex ${ }^{\circledR}$ family of FPGAs from Xilinx Incorporated support multiple FFT cores with numerous FFT implementation options [24]. In an effort to restrict the application to one Virtex ${ }^{\circledR} 5$ FPGA [25], an FFT size of 32768 points was selected.

For all simulations, a 32768 point FFT was employed. Therefore, the minimum signal separation, based on a minimum $12.5 \mathrm{KHz}$ separation, is 81.92 bins. Due to the large signal separation, the requirement of a 4 bin signal separation is achieved. When the input signal is sufficiently windowed, the performance of the multiple sinusoid estimates should approach those of the single sinusoid case.

\subsection{Detection Results}

The periodogram detection results are grouped into four simulation types based on input signal conditions. The simulation types are summarized as follows:

- Single sinusoid at center of an FFT bin;

- Single sinusoid with random frequency, uniformly distributed within one FFT bin;

- Single sinusoid with random frequency and random amplitude; and,

- Multiple sinusoid with random frequency and random amplitude. 
For each simulation type, simulations were performed for the Bartlett and Welch methods, as well as single channel and multiple channel detection.

Each simulation is composed of 1000 independent trials, to ensure statistically reliable results.

For each trial, two input signals were generated, the first consisting of only noise and the second consisting of signal(s) plus noise. The noise only data was used to evaluate the probability of false alarm for the given test conditions, while the signal plus noise data was used to evaluate the probability of detection.

For each trial, each input sinusoid was assigned a random phase and bearing. The assigned phase and bearing values were uniformly distributed between 0 and $2 \pi$.

\subsubsection{Single Sinusoid with Frequency at Center Bin}

The center bin results represent the best possible performance for the detectors. When the signal is located at the center of an FFT bin, the signal is not degraded by scalloping loss. Therefore, it demonstrates the maximum signal to noise ratio achievable for the selected FFT parameters.

The frequency selected for the single sinusoid, center bin simulations was 58.75 $\mathrm{MHz}$, corresponding to frequency bin 8192 .

\section{Single Channel Detection}

Single channel detection performance was evaluated using channel 1 of the simulated antenna array.

For the Bartlett method, the one second of input data was segmented into 152 non-overlapping blocks of 32768 samples. The single channel detection results, for the Bartlett method and a single sinusoid at the center of an FFT bin, are shown in 
Figure 4.1.

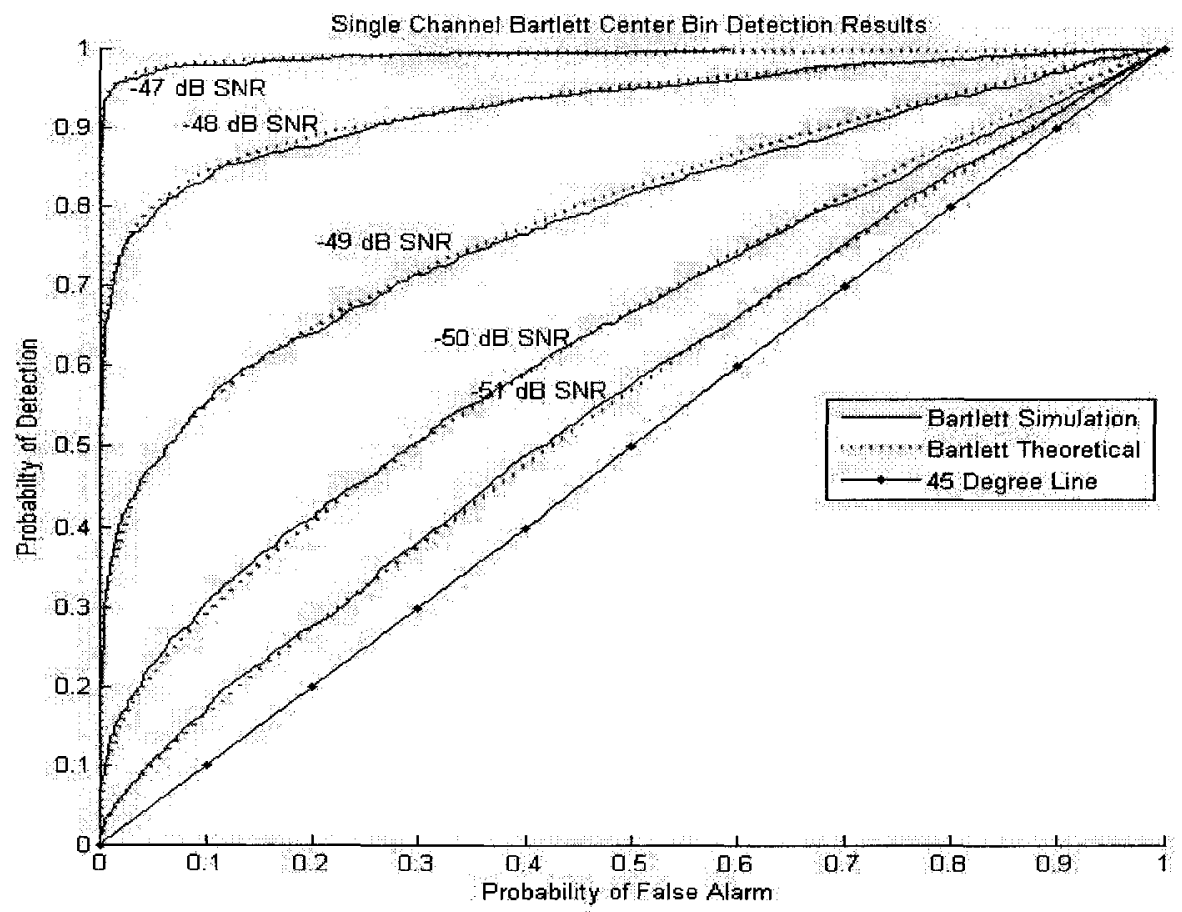

Figure 4.1: ROC for single channel Bartlett detection with frequency at center of FFT bin.

As indicated in Figure 4.1, the Bartlett simulation results are in very good agreement with the theoretical results derived in Section 2.3.2. 
For the Welch method, the one second of data was segmented into 304 overlapping blocks of length 32768 samples. The samples were segmented using $50 \%$ overlap and each block was windowed using a 32768 point Hann window.

The single channel results, for a single sinusoid at the center of an FFT bin using the Welch method, are presented in Figure 4.2. 


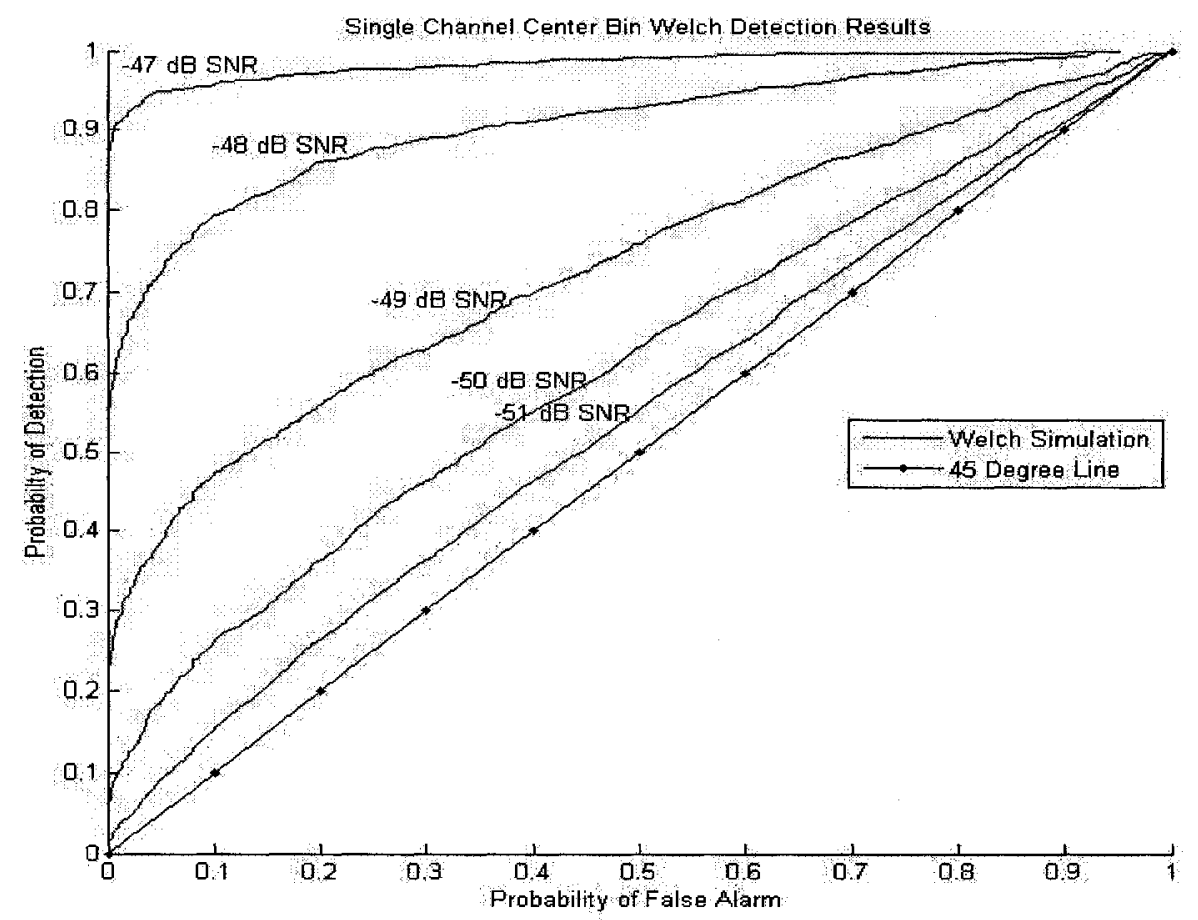

Figure 4.2: ROC for single channel Welch detection with frequency at center of FFT bin.

For the case of a single sinusoid located at the center of an FFT bin, the Bartlett method outperforms the Welch method as expected. A comparison of the two single channel methods is presented in Figure 4.3. 


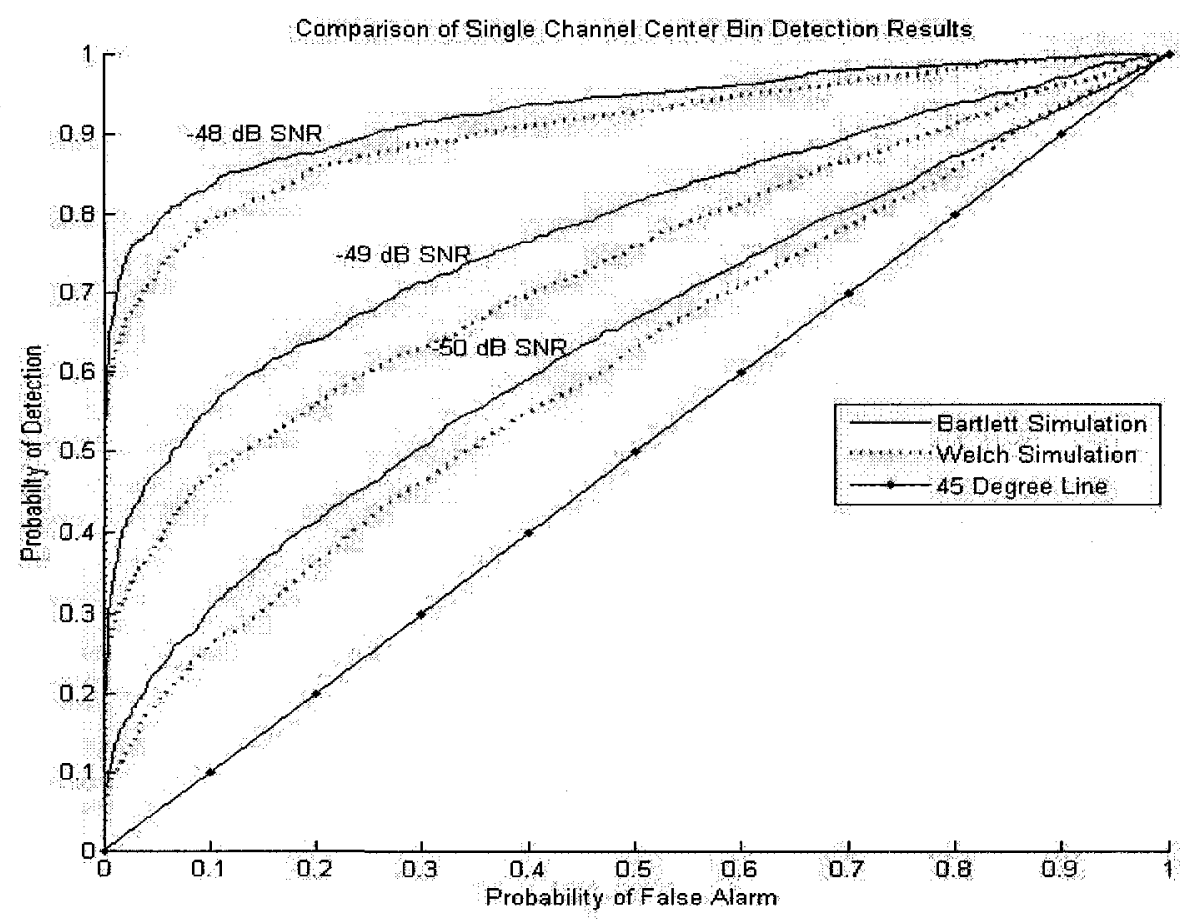

Figure 4.3: Comparison of ROCs for single channel Bartlett and Welch detection with frequency at center of FFT bin.

\section{Multiple Channel Detection}

The multiple channel detection was performed by processing four individual channels and then averaging the results to further reduce the variance of the periodogram estimates.

The multiple channel results, for the Bartlett method, with a single sinusoid at the center of an FFT bin, are shown in Figure 4.4.

As the results indicate, the multiple channel Bartlett simulations are in very good agreement with the theoretical performance. 


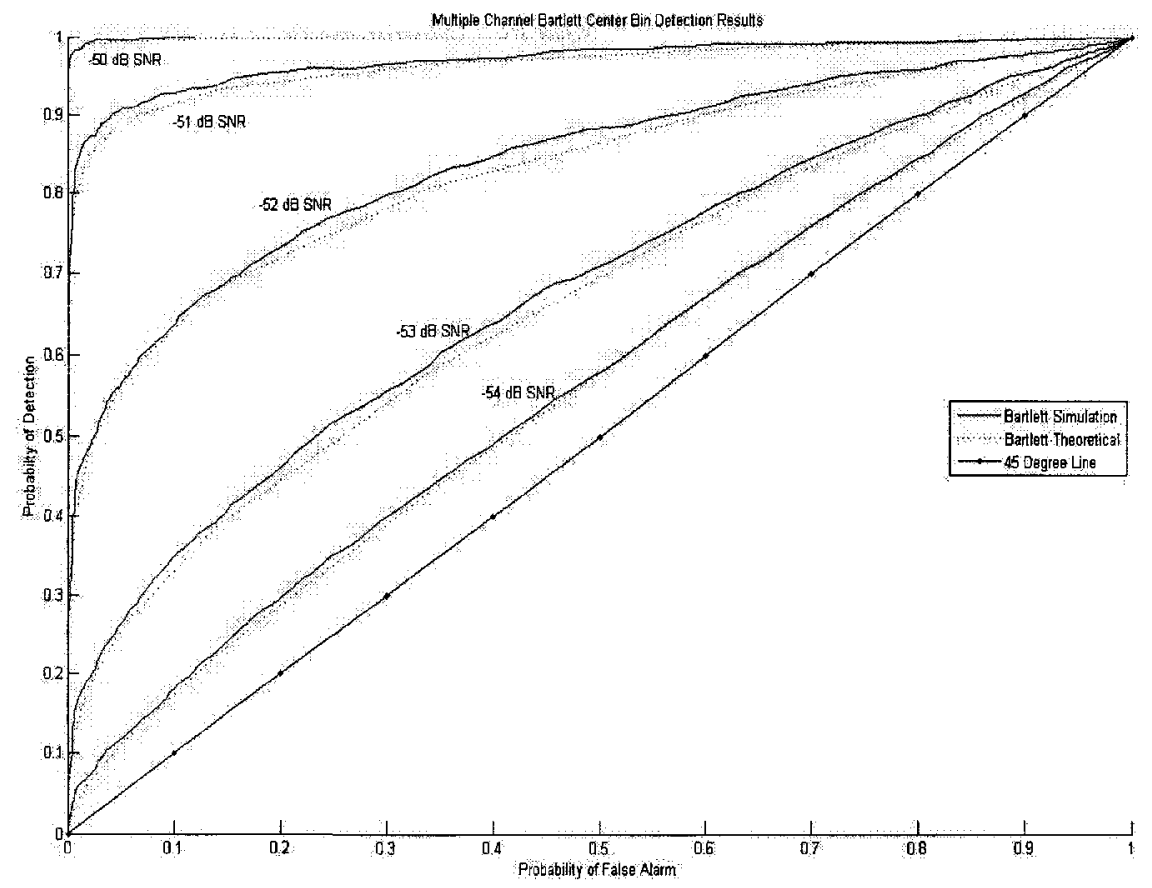

Figure 4.4: ROC for multiple channel Bartlett detection with frequency at center of FFT bin.

For the multiple channel Welch method, the detection performance for a single sinusoid centered on an FF' bin is presented in Figure 4.5. 


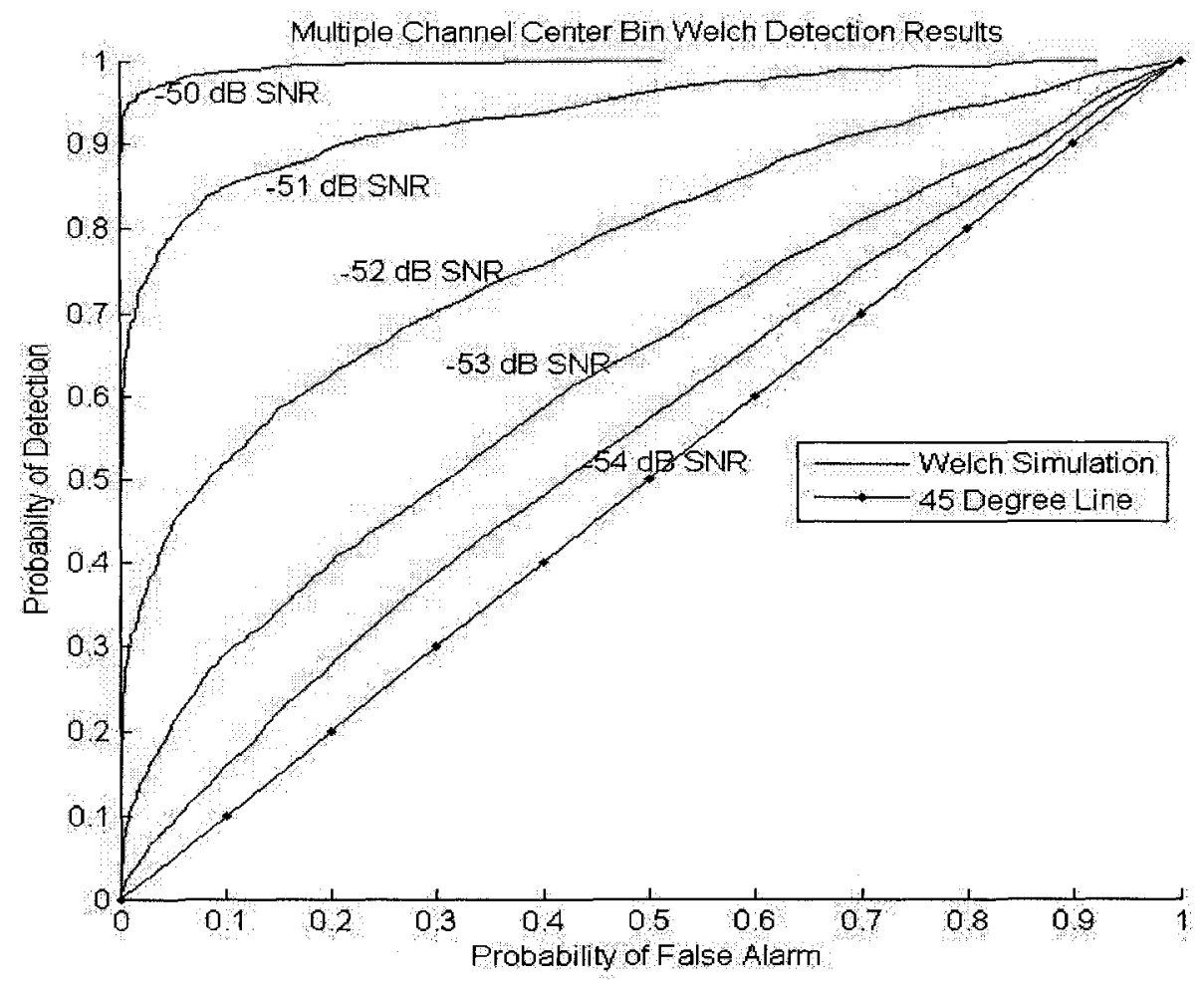

Figure 4.5: ROC for multiple channel Welch detection with frequency at center of FFT bin.

As with the single channel detectors, the multiple channel Bartlett method outperformed the multiple channel Welch method, when the sinusoid is centered on an FFT bin. The comparison of the multichannel detectors is shown in Figure 4.6. 


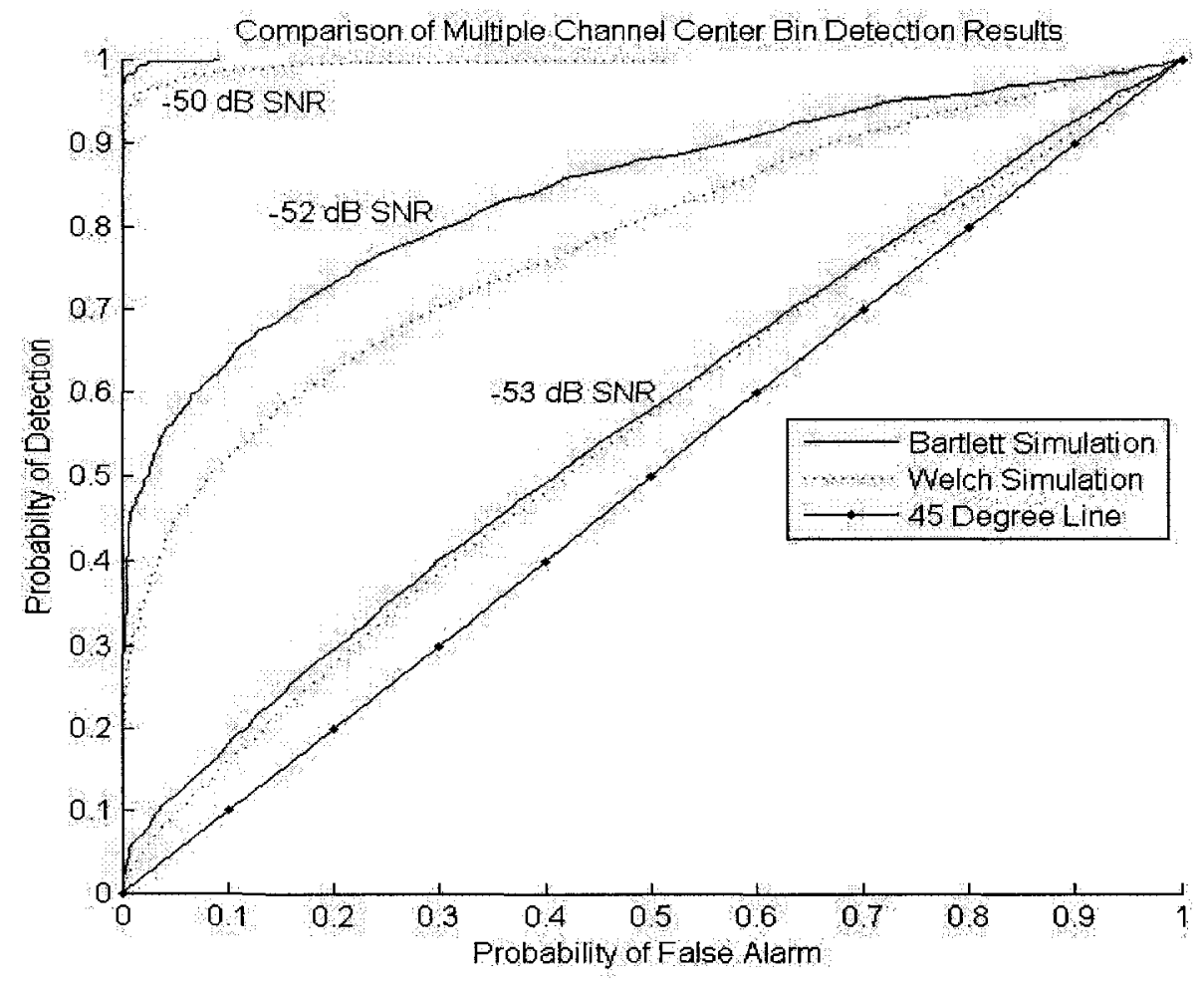

Figure 4.6: Comparison of ROCs for multiple channel Bartlett and Welch detection with frequency at center of FFT bin.

The multiple channel detectors outperformed the single channel detectors by approximately $3 \mathrm{~dB}$. The $3 \mathrm{~dB}$ processing gain is due to the non-coherent combining of the four independent channels. The performance gain of the multiple channel detectors versus the single channel detectors is shown in Figure 4.7 . 


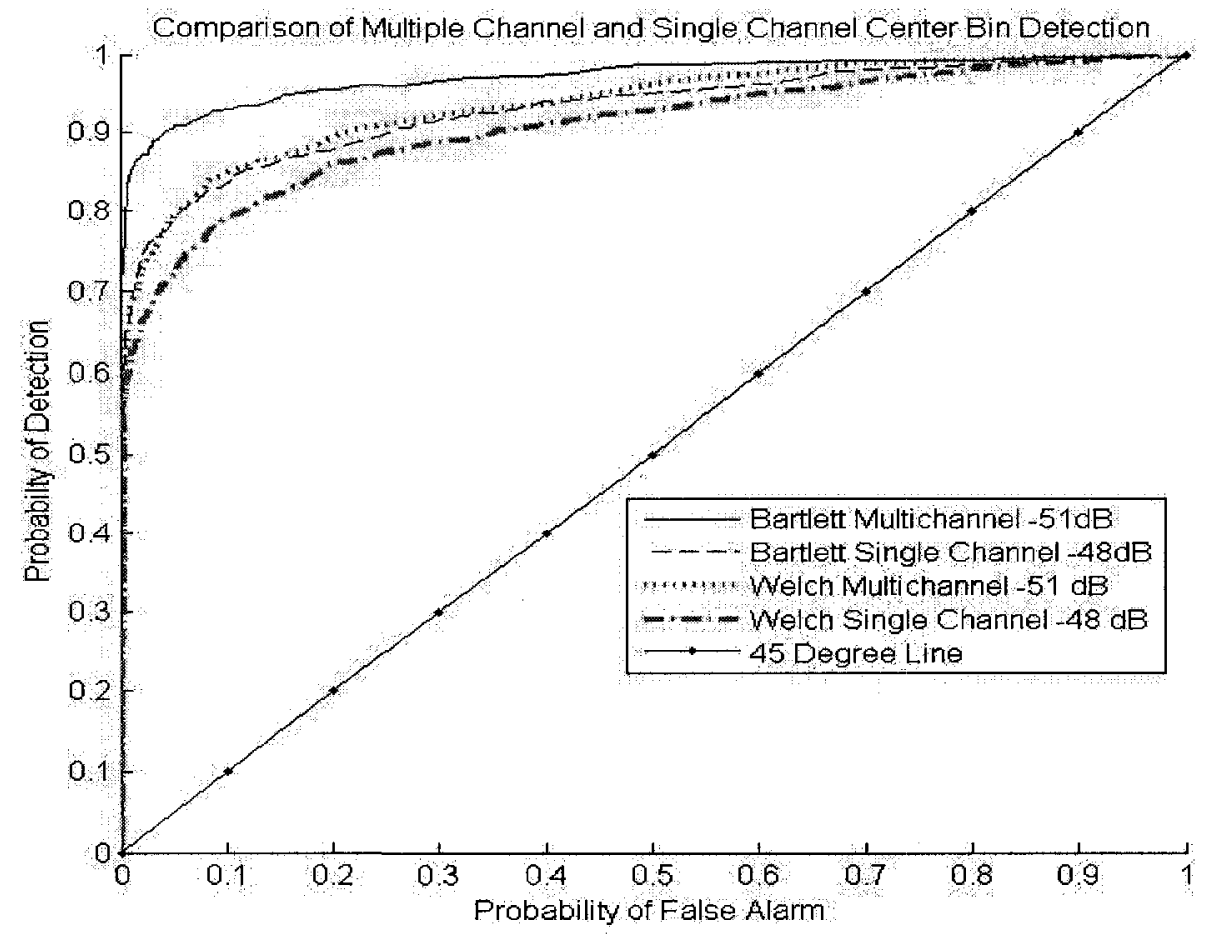

Figure 4.7: ROC for single channel Welch detection with frequency at center of FFT bin. 


\subsubsection{Single Sinusoid with Random Frequency}

It many practical applications, the frequency of the target signal is unknown. As a result, the sinusoid can be located at any position within an FFT bin. When the sinusoid is offset from the center of an FFT bin, the window function and its associated scalloping loss will have a negative impact on signal detection performance.

The simulations in this section evaluate the detection performance for the case of a single sinusoid with random frequency. To ensure an even distribution, the frequencies were selected from a uniform distribution within frequency bin 8192 .

\section{Single Channel Detection}

The single channel, Bartlett detection results for the case of a single sinusoid with random frequency are presented in Figure 4.8.

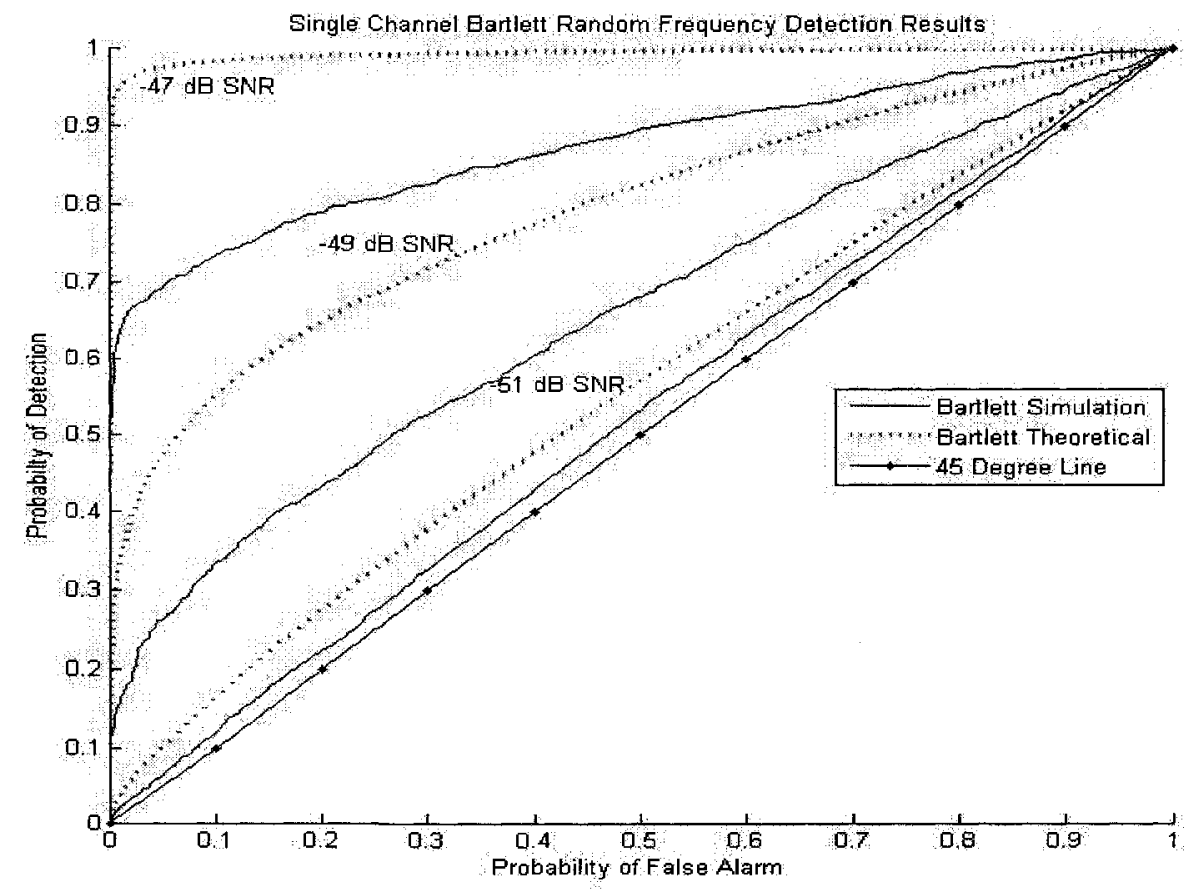

Figure 4.8: ROC for single channel Bartlett detection with random frequency. 
As a reference, the Bartlett theoretical results from the previous section (center bin) have been provided. The simulation results indicate significant performance degradation when the sinusoid frequency deviates from the center of the FFT bin. The Bartlett method does not employ windowing, which is equivalent to rectangular windowing in the time domain. The resulting sinc response in the frequency domain has a narrow main lobe ( $3 \mathrm{~dB}$ bandwidth of 0.89 bins) and considerable scalloping loss $(3.92 \mathrm{~dB})[26]$, resulting in considerable spectral leakage and reduced signal energy within the target frequency bin. The effect of the rectangular window on detection performance is observed in Figure 4.8 and is appreciable.

The single channel, Welch detection results for the case of a single sinusoid with random frequency are presented in Figure 4.9.

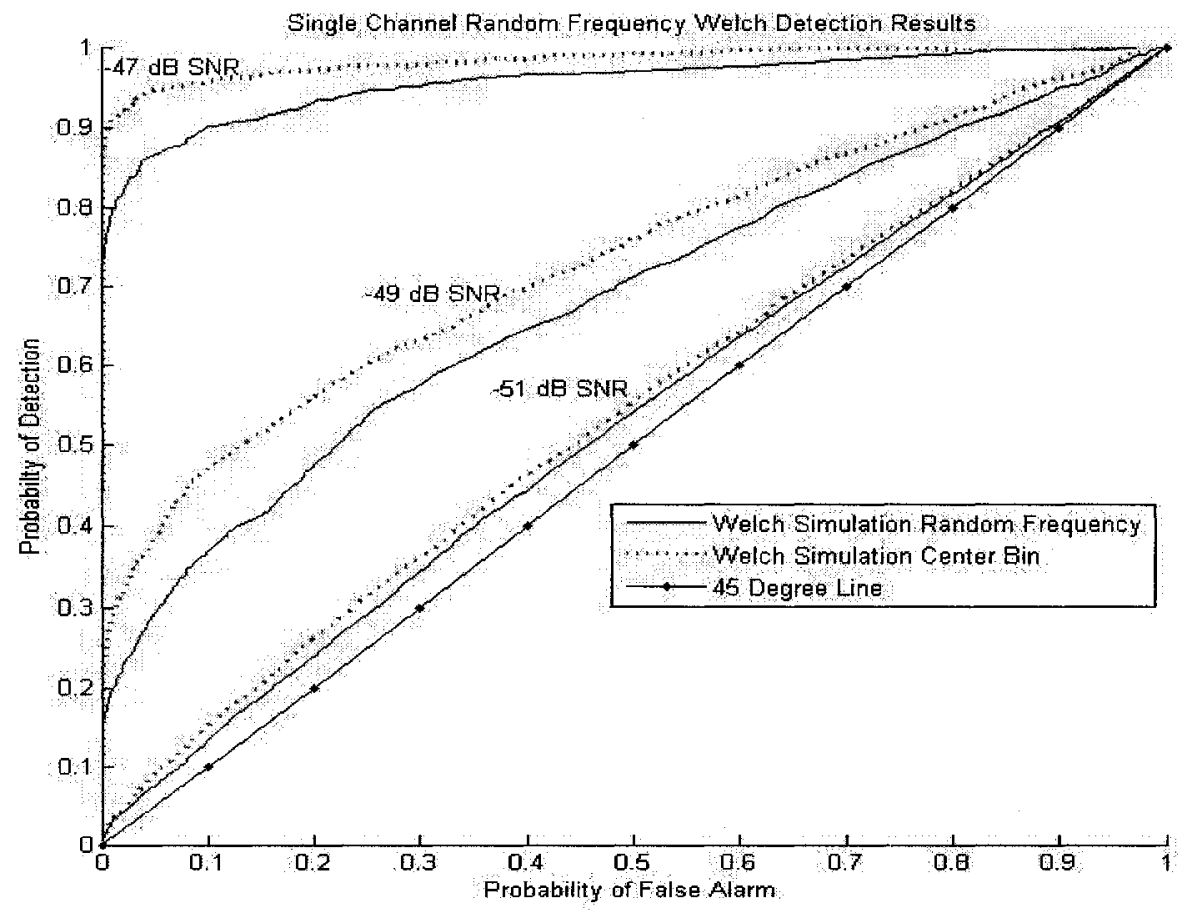

Figure 4.9: ROC for single channel Welch detection with random frequency. 
As a reference, the Welch simulation results from the previous section (center bin) have been provided. The results indicate that the Welch method does not experience the same degree of performance degradation as the Bartlett method, when the frequency is randomized. The frequency domain response of the Hann window has a broader main lobe ( $3 \mathrm{~dB}$ bandwidth of 1.44 bins) and less scalloping loss (1.42 $\mathrm{dB}$ ) [26], resulting in less spectral leakage and more signal energy within the target frequency bin. As a result, the Welch method exhibits much lower performance degradation for sinusoids with random frequencies.

A comparison of the detection performance for the single channel Bartlett and Welch Methods is provided in Figure 4.10.

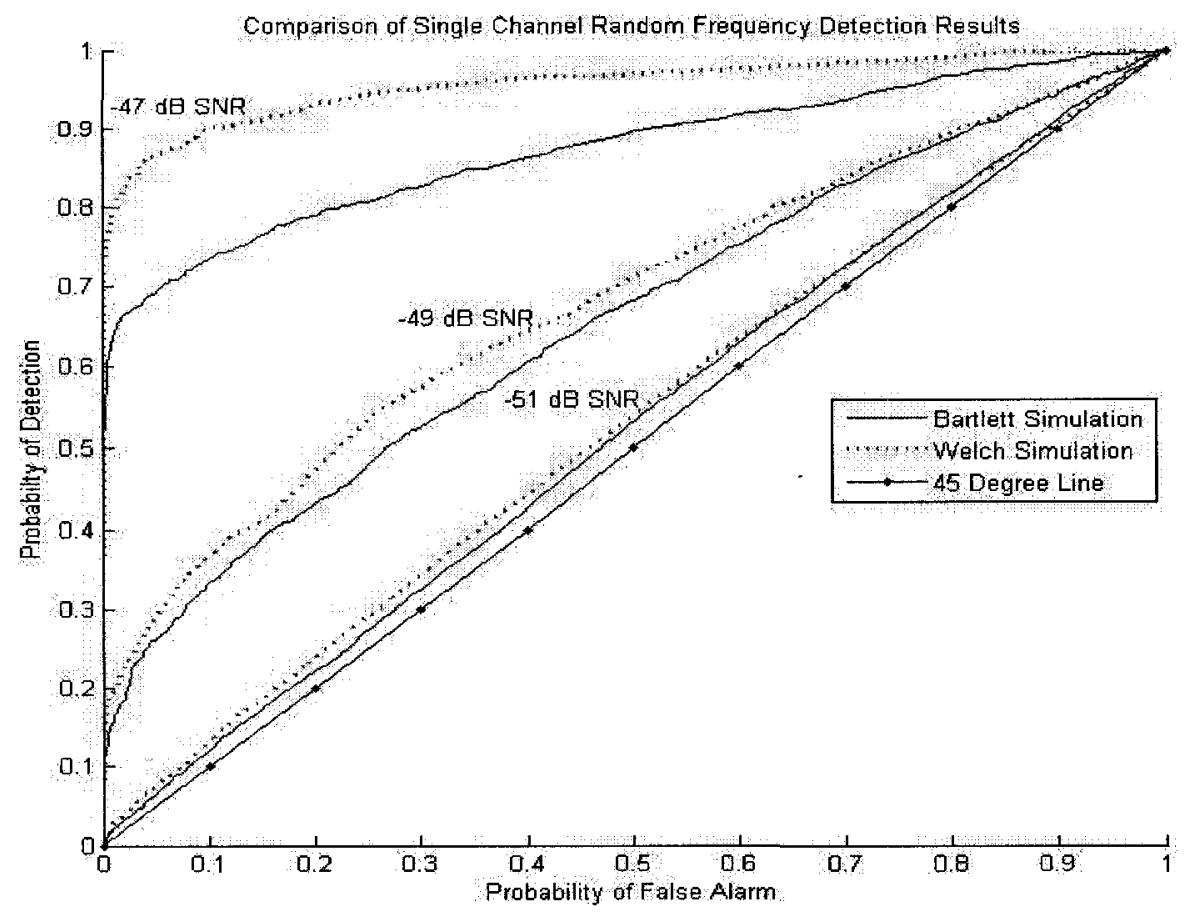

Figure 4.10: ROC comparison for single channel detection with random frequency.

As the results indicate, the single channel Welch method outperforms the single 
channel Bartlett method for the case of single sinusoid with random frequency. The three spectral averaging variables influencing detection performance are FFT size, percentage overlap and the window function employed. To constrain the computational complexity to a single Virtex ${ }^{\circledR} 5$ FPGA, the FFT size was fixed at 32768 points for all simulations. Therefore, the change in relative performance for the Bartlett and Welch methods can be attributed to percentage overlap and the window function employed. The Hann window function of the Welch technique has a wider main lobe and lower worst case processing loss in comparison with the rectangular window of the Bartlett technique. As a result, the Hann window experiences less SNR degradation when the signal frequency deviates from the center of an FFT bin. The $50 \%$ overlap of the Welch method implemented in this thesis also contributes to the observed performance improvement (i.e. The $50 \%$ overlap increases the number of non-coherent averages by approximately a factor of 2 ).

\section{Multiple Channel Detection}

The multiple channel detection was performed by processing four individual channels and averaging the results to further reduce the variance of the periodogram estimates.

The multiple channel results, for the Bartlett method and the Welch method are shown in Figure 4.11. 


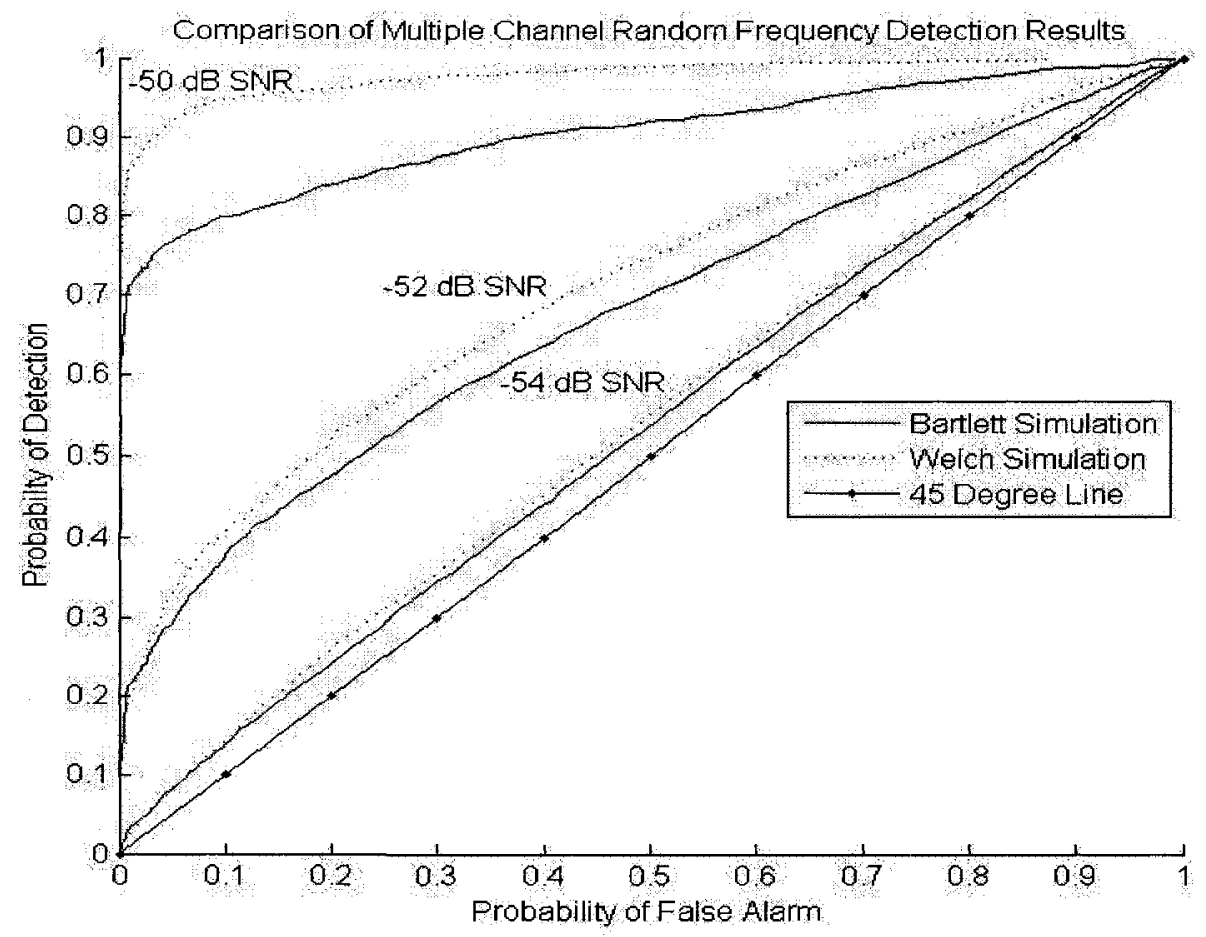

Figure 4.11: ROC comparison for multiple channel detection with random frequency.

In figure 4.11 , a $3 \mathrm{~dB}$ processing gain is realized by averaging the four independent channels. This result is consistent with the $3 \mathrm{~dB}$ gain observed in the center bin results of section 4.1.1. A comparison of the single channel and multiple channel detection performance, for the case of a single sinusoid with random frequency is shown in Figure 4.12. 


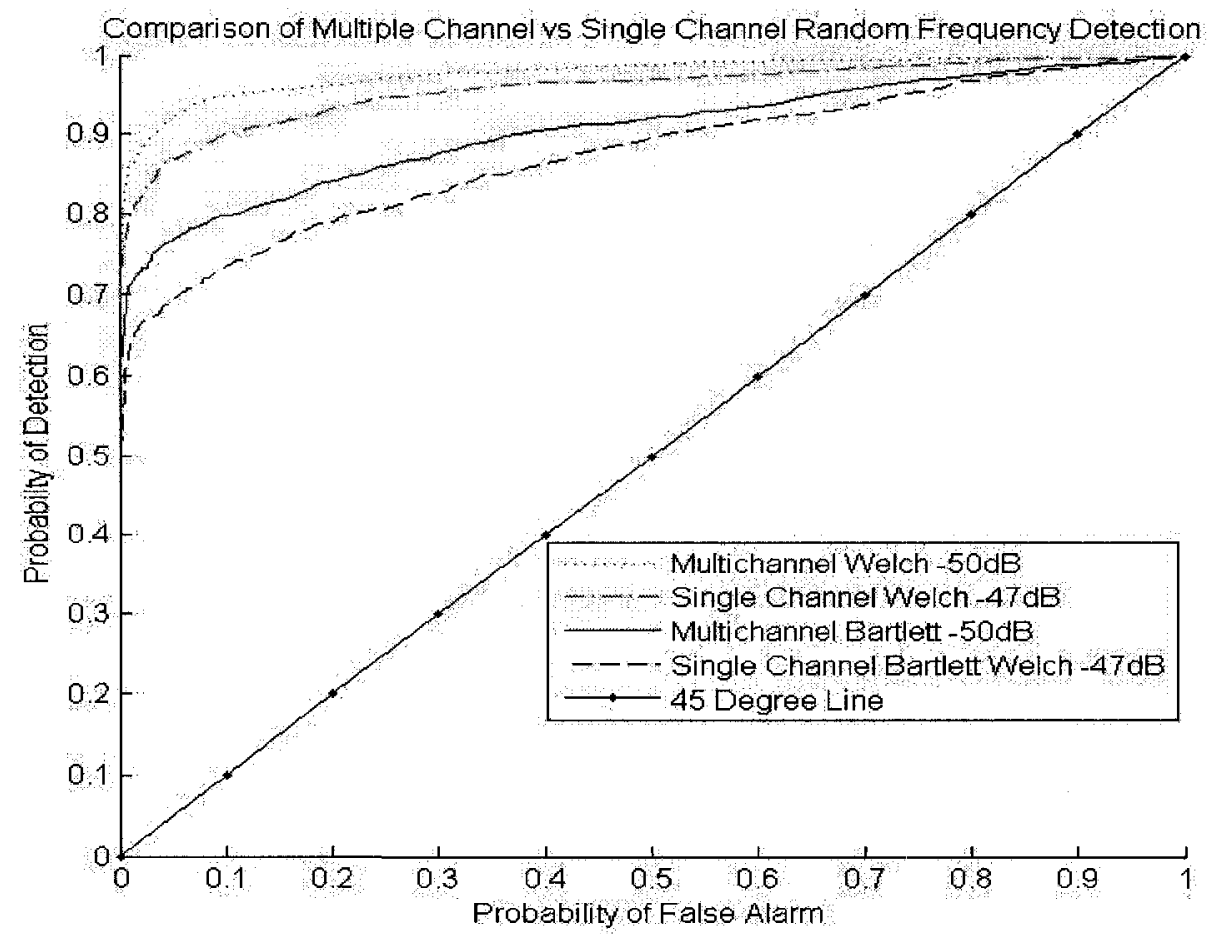

Figure 4.12: ROC comparison single vs. multiple channel detection with random frequency. 


\subsubsection{Single Sinusoid with Random Frequency and Random Amplitude}

In many practical applications, the amplitude of the target signal is also unknown. However, the signal amplitude may be confined to a known range and the detection performance will be bounded by the fixed amplitude performance for the bounds of the amplitude range.

The simulations in this section build upon those in the previous section. A single sinusoid was generated with uniformly distributed phase (0 to $2 \pi$ ), bearing ( 0 to $2 \pi$ ) and frequency (uniform within FFT bin 8192).

\section{Single Channel Detection}

For the single channel detection simulations, the SNR of the sinusoid was uniformly distributed over the range -47 to $-51 \mathrm{~dB}$. The single channel detection performance of the Bartlett and Welch method for a sinusoid with random frequency and random amplitude is shown in Figure 4.13

As the results indicate, the fixed amplitude results for the limits of the amplitude range provide coarse bounds for the random amplitude detection performance. Simulations were performed for the amplitude ranges 35 to $45 \mathrm{~dB}$ and 45 to $50 \mathrm{~dB}$, producing similar results.

As observed in the fixed amplitude case, the Welch method outperformed the Bartlett method.

Also of note, the detection performance of both detection methods for random amplitude, were slightly higher than their corresponding fixed amplitude results for the mean of the amplitude range $(-49 \mathrm{~dB})$. 


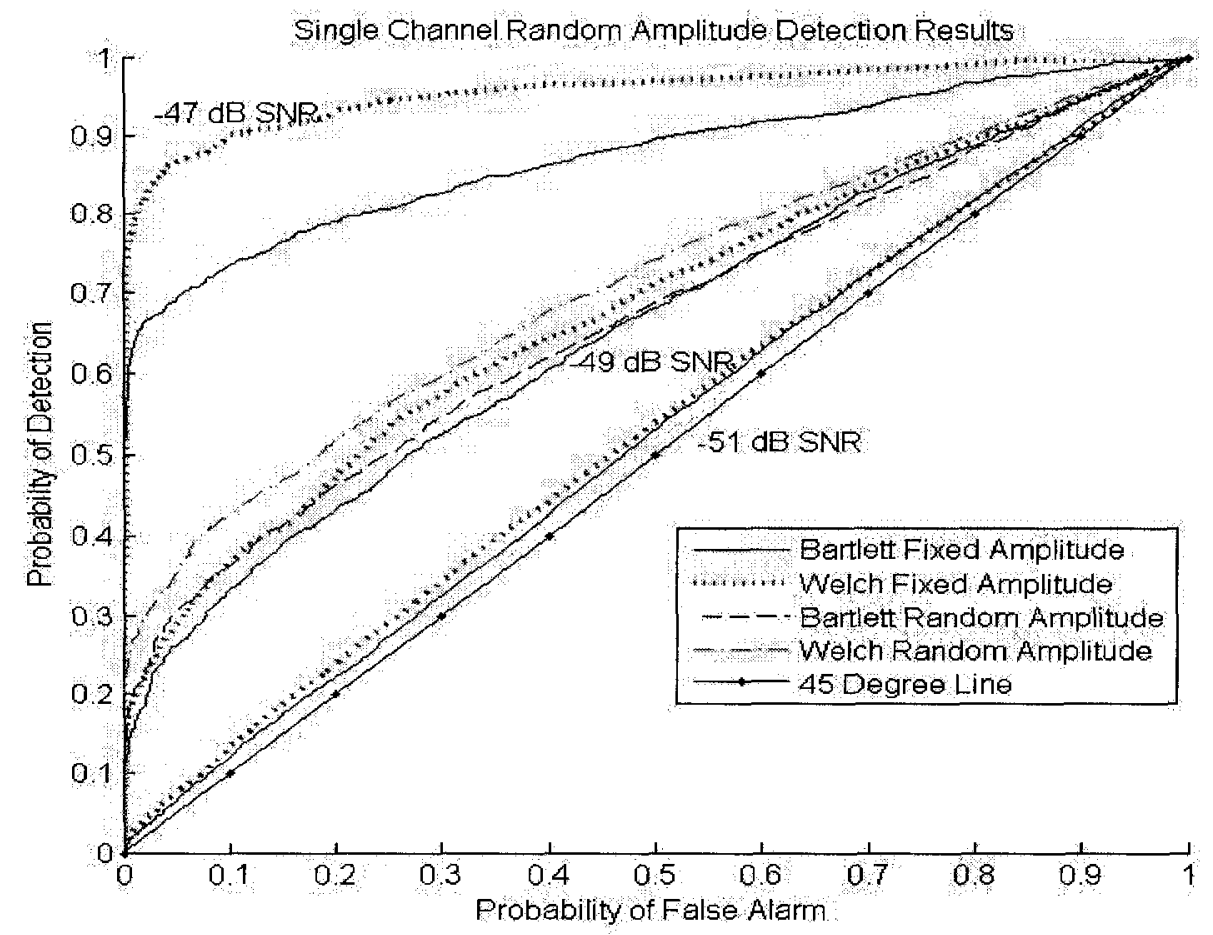

Figure 4.13: ROC for single channel detection with random frequency and random amplitude. 


\section{Multiple Channel Detection}

The multiple channel detection was performed by processing four individual channels and averaging the results to further reduce the variance of the periodogram estimates.

The multiple channel results, for the Bartlett method and the Welch method are shown in Figure 4.14

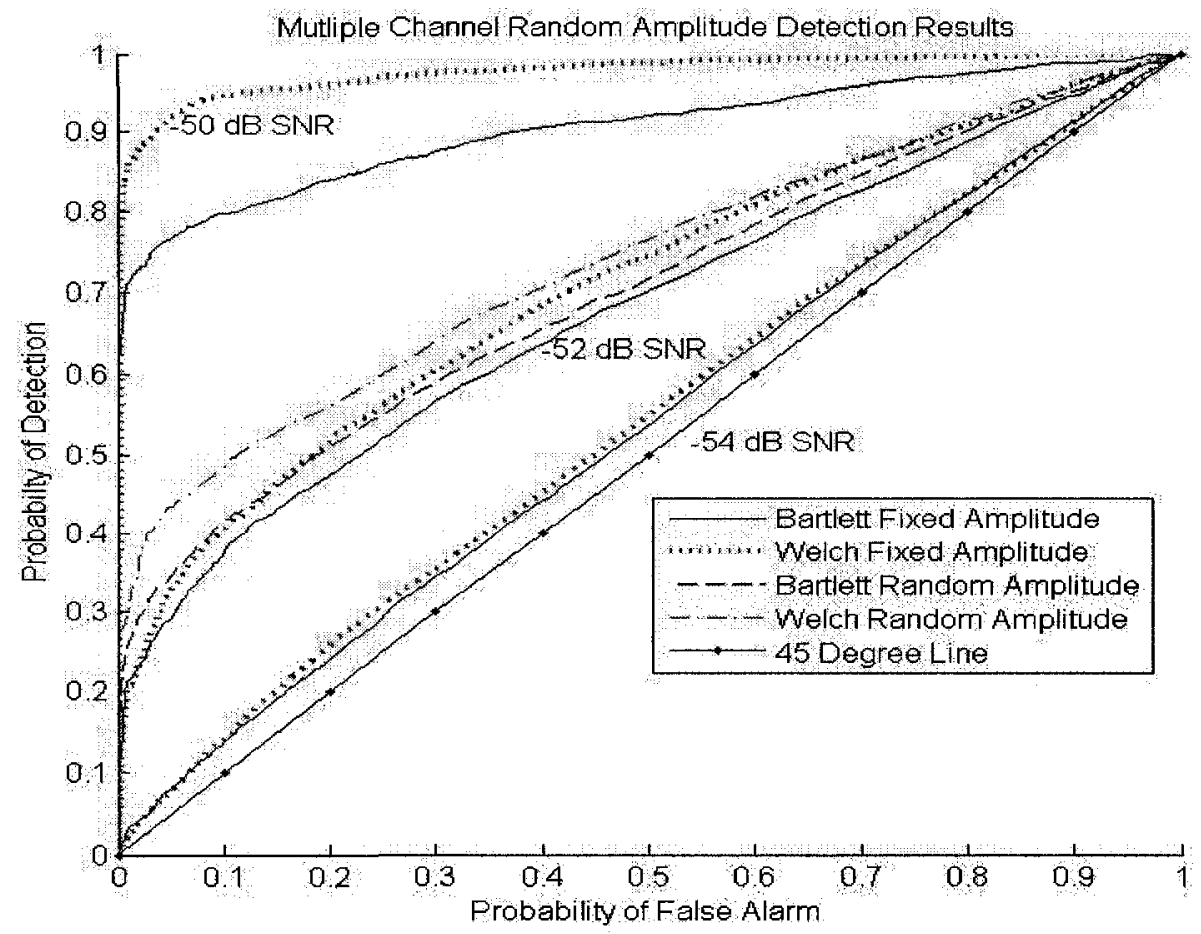

Figure 4.14: ROC for multiple channel detection with random frequency and random amplitude.

Similar to the single channel results, the multiple channel detection performance is coarsely bounded by the fixed amplitude performance at the amplitude bounds. Additionally, the detection performance is slightly greater than the fixed amplitude performance at the mean of the amplitude range $(-52 \mathrm{~dB})$.

A comparison of the single channel and multiple channel detection performance is shown in Figure 4.15. As observed in the previous sections, the multiple channel 
detection provides about $3 \mathrm{~dB}$ of processing gain.

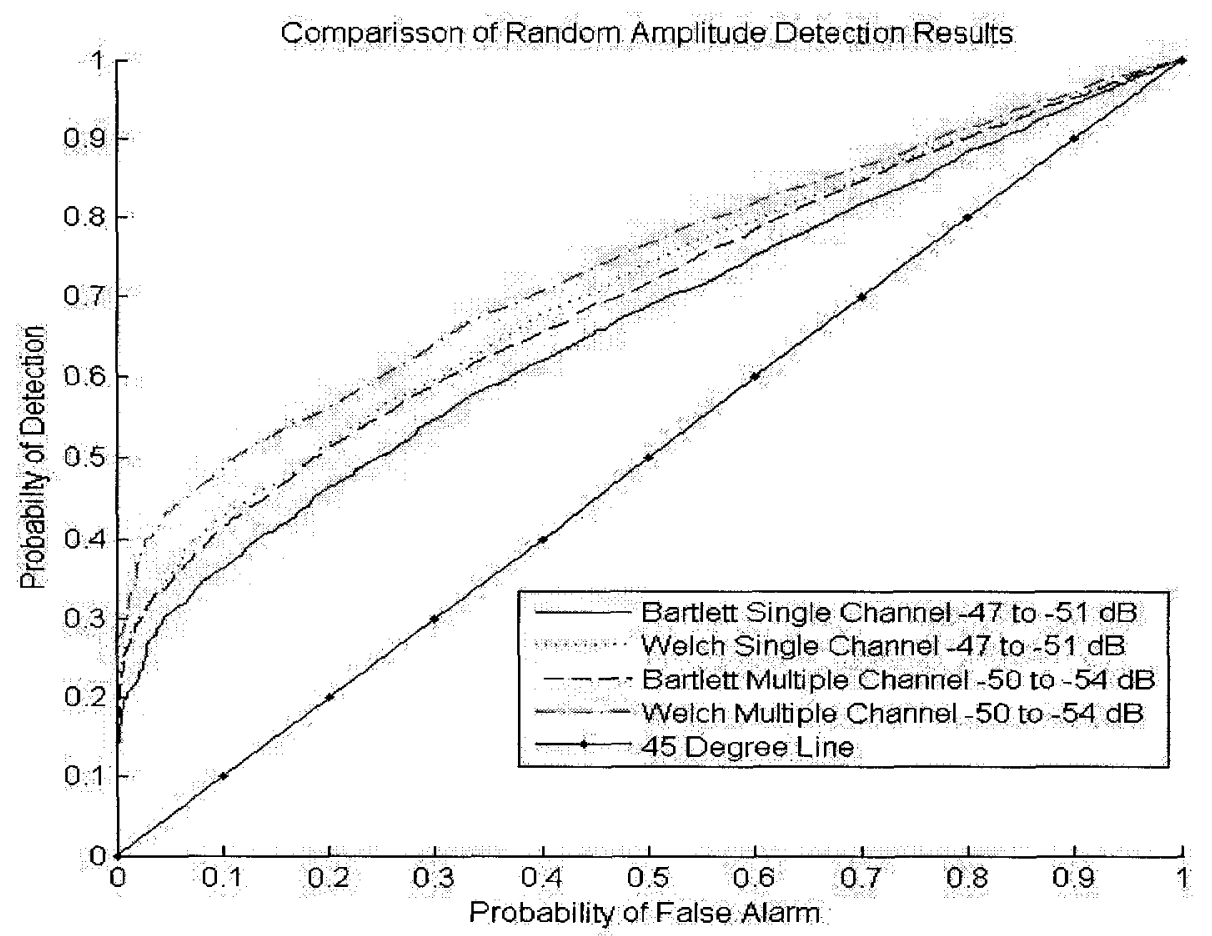

Figure 4.15: Comparison of ROCs for random frequency and random amplitude detection.

\subsubsection{Multiple Sinusoids with Random Frequency}

The multiple sinusoid detection performance was evaluated by simulating 10 sinusoids of random phase and bearing, with a minimum frequency separation of $12.5 \mathrm{kHz}$.

As discussed in Section 2.3.3, the standard ROC curve presents a biased representation of detection performance when multiple sinusoids are present. Instead, the probability of detection curve is calculated for all sinusoids present. The multiple sinusoid probability of detection is compared with that of the single sinusoid case, to evaluate the performance degradation due to the presence of multiple sinusoids. 


\section{Single Channel Detection}

The single channel detection performance was evaluated by uniformly distributing the SNR of the 10 sinusoids from -47 to $-51 \mathrm{~dB}$. The single channel, multiple sinusoid detection results are presented in Figure 4.16. The multiple sinusoid detection results

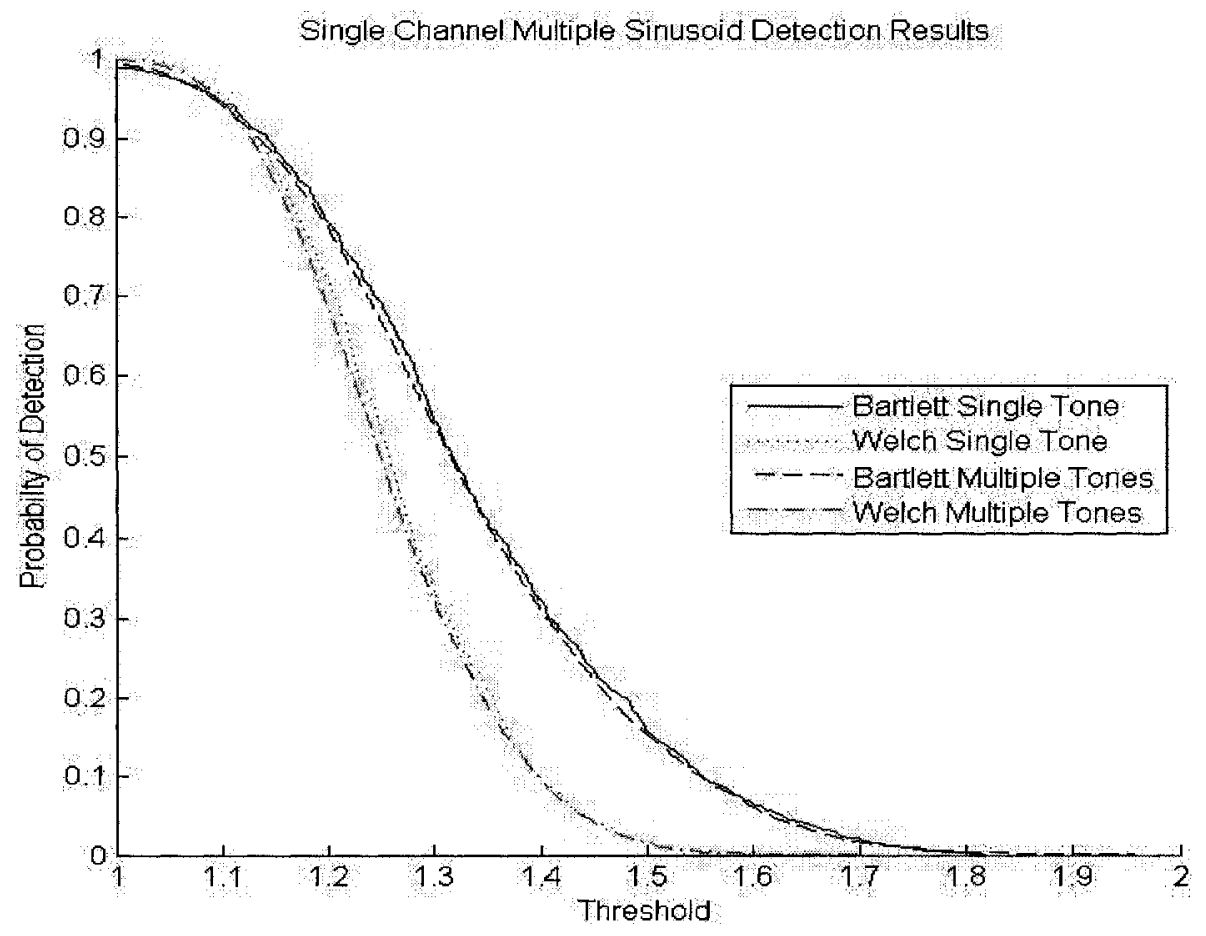

Figure 4.16: ROC comparison for single channel detection with multiple sinusoids of random frequency and random amplitude.

are in good agreement with the single sinusoid case. The minimum sinusoid frequency separation of 81.92 FFT bins provides sufficient separation, reducing the impact of spectral leakage to a negligible level. Therefore, the single sinusoid performance bounds can be applied to the multiple sinusoid case, for the specified signal conditions. 


\section{Multiple Channel Detection}

The multiple channel detection performance was evaluated by uniformly distributing the SNR of the 10 sinusoids from -50 to $-54 \mathrm{~dB}$. The multiple channel results, for the Bartlett method and the Welch method are shown in Figure 4.17

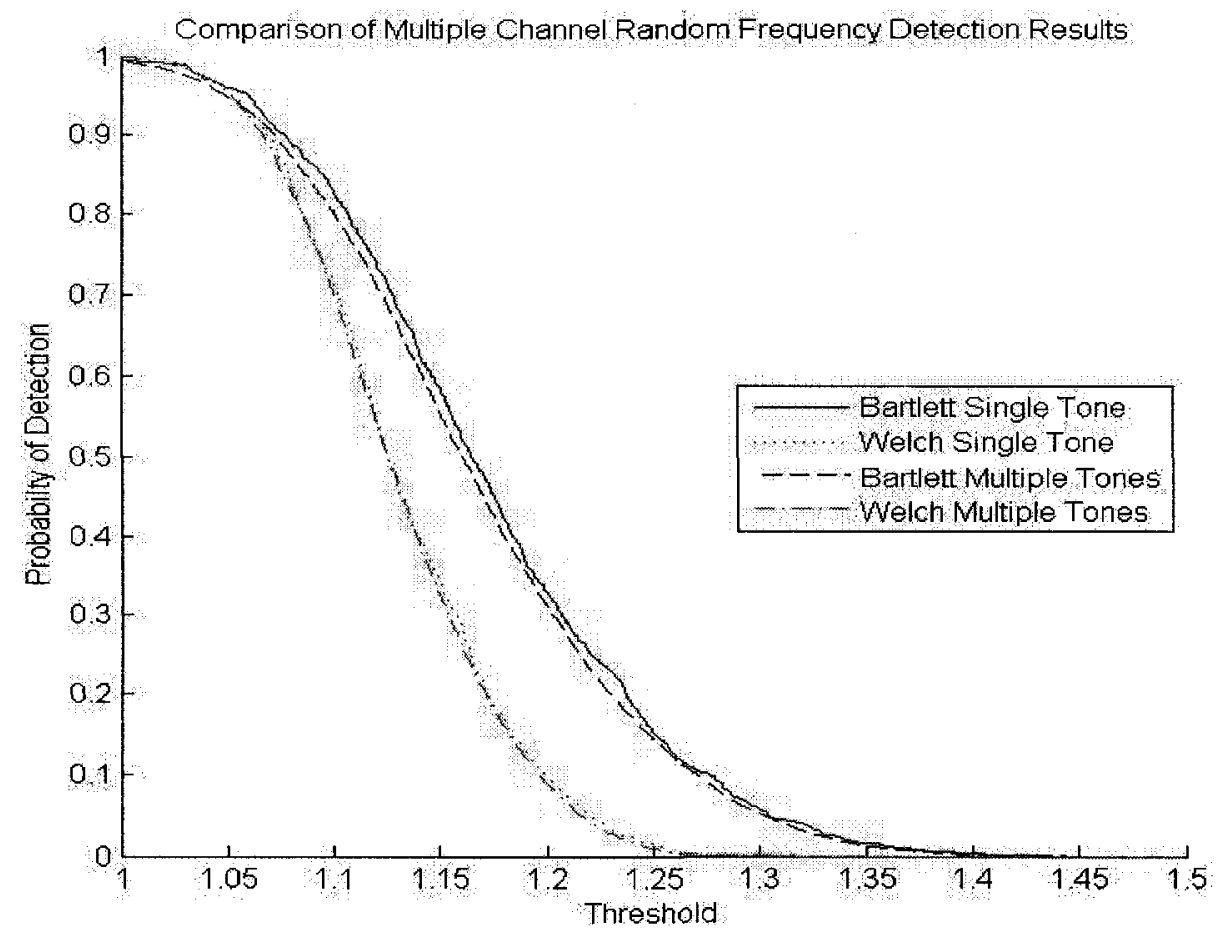

Figure 4.17: ROC comparison for multiple channel detection with multiple sinusoids of random frequency and random amplitude.

The multiple sinusoid detection results are in good agreement with the single sinusoid case for multiple channels, as was also the case for single channel detection. Therefore, the single sinusoid, multiple channel performance bounds can be used to predict the detection performance, when multiple sinusoids are present. 


\subsubsection{Conclusions}

The simulation results for the case of a single sinusoid, at the center of an FFT bin, were in very good agreement with the theoretical values. Additionally, the Bartlett method demonstrated superior performance when the sinusoids were located at the center of an FFT bin.

For the case of a single sinusoid with random frequency, both the Bartlett and Welch methods experienced a performance degradation. However, the performance impact was considerably greater with the Bartlett method and the Welch method demonstrated much better overall performance. The most likely factor influencing this change in detection performance is the window function employed by the different techniques.

For the case of a single sinusoid with random amplitude and random frequency, the detection performance was coarsely bounded by the fixed amplitude detection performance at the bounds of the amplitude range. Additionally, the detection performance was observed to be slightly greater than the fixed amplitude performance at the mean of the amplitude range in decibels.

For the case of multiple sinusoids, a minimum frequency separation of 81.92 FFT bins was employed. This separation minimized the spectral leakage and the multiple sinusoid performance was observed to be in good agreement with the single sinusoid results. Therefore, the single sinusoid detection performance bounds can be used.

Averaging the four independent channels enabled non-coherent combining, resulting in a $3 \mathrm{~dB}$ processing gain. The $3 \mathrm{~dB}$ processing gain was observed in all four simulation scenarios.

For the general case of multiple sinusoids with unknown amplitude, frequency and phase, the multichannel Welch technique is the recommended approach. 


\subsection{Bearing Estimation Results}

The bearing estimation results are grouped into three simulation scenarios based on input signal conditions. The simulation scenarios are summarized as follows:

- Single sinusoid at center of an FFT bin;

- Single sinusoid with random frequency; and,

- Multiple sinusoids with random frequency and random amplitude.

For each simulation type, simulations were performed for the Bartlett and Welch methods, as well as interferometer and Eigen-decomposition bearing estimation.

Each point on the bearing estimation performance curve represents the mean of 250 independent trials.

The bearing estimation results are further subdivided based on the bearing of the desired signal $\left(0, \frac{\pi}{8}\right.$ and $\left.\frac{\pi}{4}\right)$. For each simulation type, bearing estimation performance was measured by the bearing RMSE in radians.

For each trial, each input sinusoid was assigned a random phase. The assigned phase values were uniformly distributed between 0 and $2 \pi$.

The CRLB for bearing estimation was provided in Section 2.4.1, where the Euclidean norm of the antenna positions was specified in equation 2.106. The squared Euclidean norm of the four element circular array, with radius one meter and maximum frequency $60 \mathrm{MHz}$ is given by:

$$
\left|\mathbf{r}_{x}\right|^{2}=\left(\frac{2(1)}{5}\right)^{2} \sum_{x=1}^{4}\left[\cos \left(\frac{2 \pi x}{4}\right)\right]^{2}=\left(\frac{2}{5}\right)^{2}(2)=0.32
$$


Substituting equation 4.1 and the FFT length of $32 \mathrm{k}$ into equation 2.105, the CRLB for the four-element antenna array and specified signal conditions is given by:

$$
\operatorname{var}(\phi) \geq \frac{1}{2\left(5 \times 10^{6}\right) \operatorname{snr} \quad \pi^{2} 0.32}=\frac{1}{3200000 \pi^{2} \mathrm{snr}}
$$

\subsubsection{Single Sinusoid with Frequency at Center Bin}

For the case of a single sinusoid at the center of an FFT bin, the sinusoid frequency of $58.75 \mathrm{MHz}$ was used, placing it at the center of FFT bin 8192 .

\section{Sinusoid with Bearing 0}

The sinusoid from bearing 0 represents the worst case of all signal conditions. This scenario is referred to as the "end fire" condition [20] when considering the antenna pair 1 and 3. The bearing estimation performance is presented in Figure 4.18.

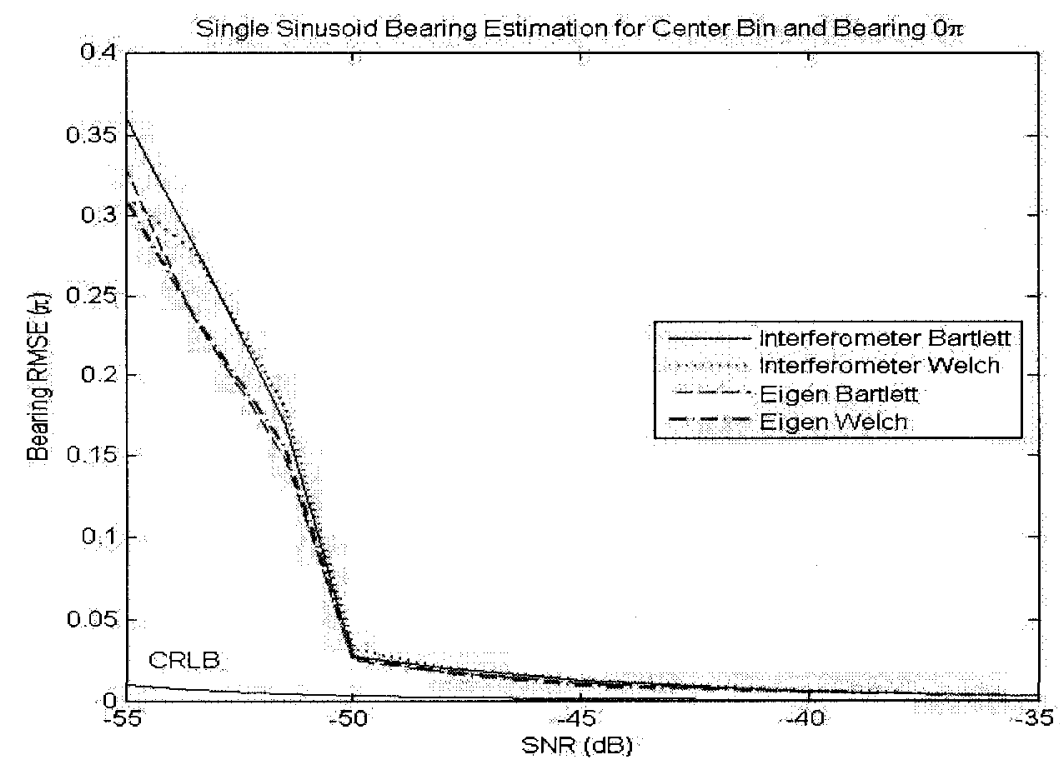

Figure 4.18: Linear representation of bearing estimation performance for a single sinusoid at the center of an FFT bin, from bearing 0 . 
As illustrated in Figure 4.18, the bearing estimation error is minimal under high SNR conditions. Additionally, at high SNRs, the bearing error approaches that of the CRLB. This result is expected, since the bearing estimation CRLB is an asymptotic bound.

The primary focus of this section is the relative performance of the interferometer and the eigen-decomposition techniques for bearing estimation. This is better demonstrated by plotting the results on a logarithmic scale as illustrated in Figure 4.19 .

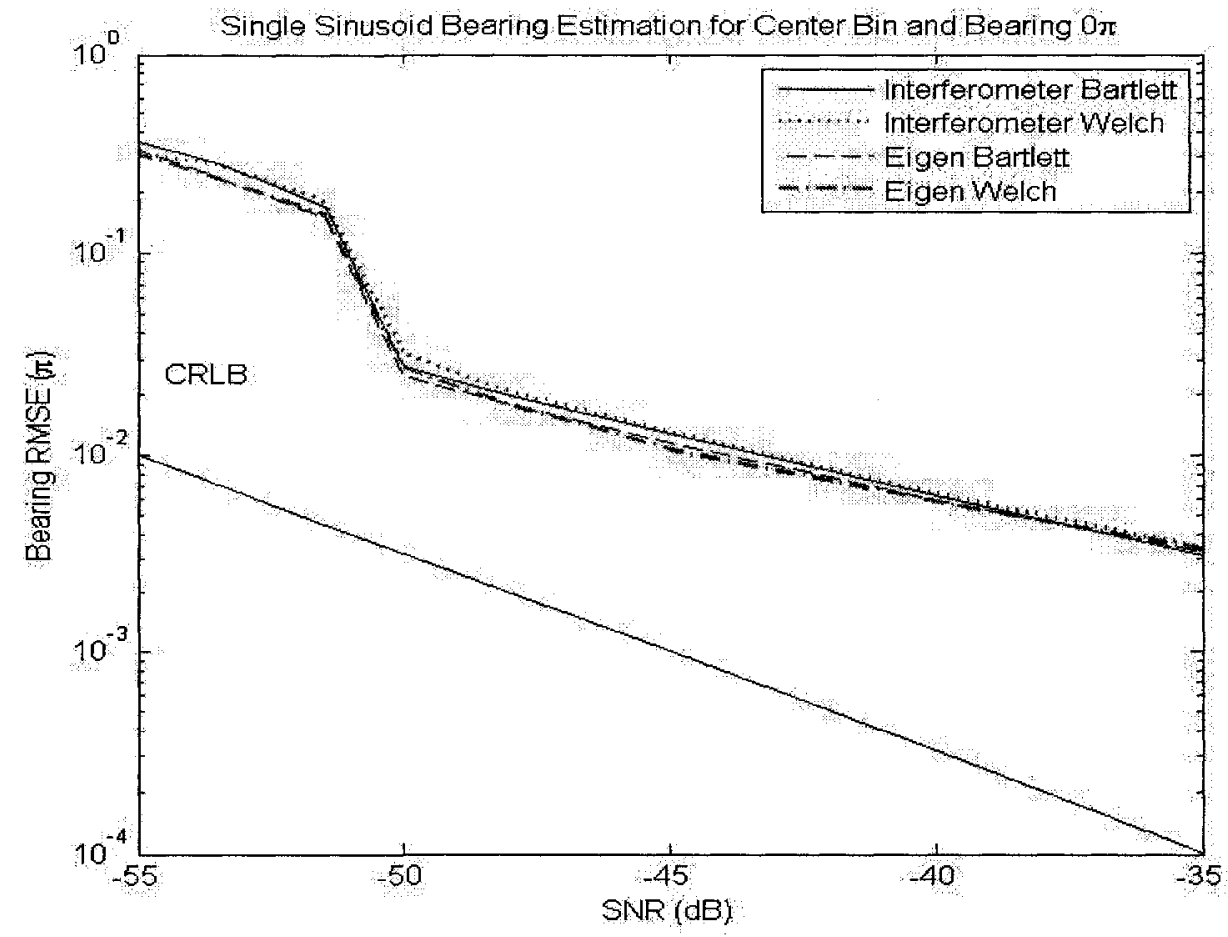

Figure 4.19: Logarithmic representation of bearing estimation performance for a single sinusoid at the center of an FFT bin, from bearing 0 .

The results indicate comparable performance for the Bartlett and Welch methods when the sinusoid is located at the center of an FFT bin. Additionally, the Eigendecomposition technique provides a slight increase in performance compared to the 
interferometer technique.

A threshold effect is observed, where below the threshold a steep rise in bearing RMSE is exhibited. The threshold effect is observed at approximately $-50 \mathrm{~dB}$ SNR for all techniques.

Additionally, the CRLB provides a loose bound on the performance of the bearing estimators. The CRLB is an asymptotic bound, providing tighter performance estimates as the SNR increases.

\section{Sinusoid with Bearing $\pi / 8$}

The sinusoid from bearing $\pi / 8$ represents an average signal condition. The bearing estimation performance is presented in Figure 4.20

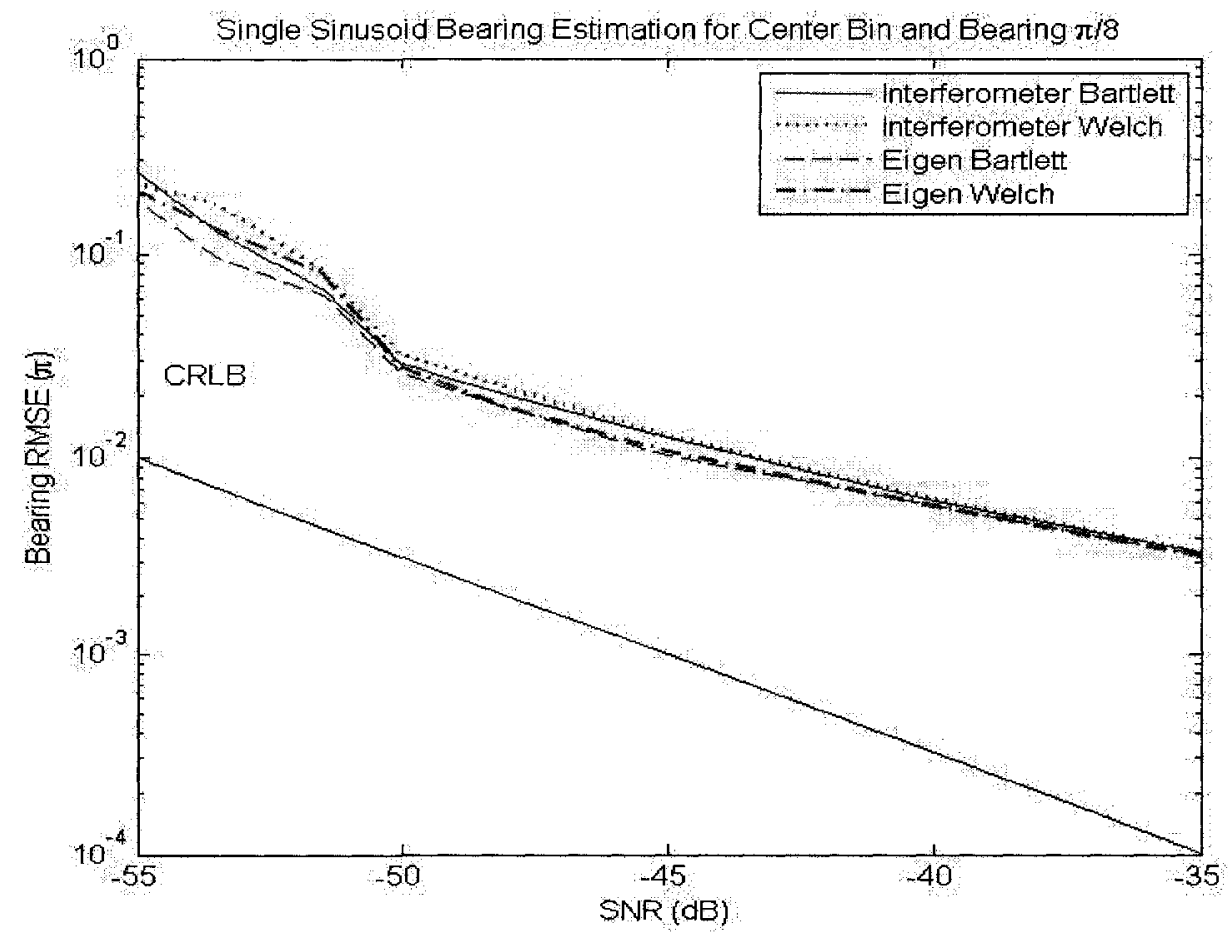

Figure 4.20: Bearing estimation performance for a single sinusoid at the center of an FFT bin, from bearing $\pi / 8$. 
The bearing estimation results indicate similar performance between the Bartlett and Welch methods. Additionally, the Eigen-decomposition technique provides a slight increase in performance compared to the interferometer technique.

For the case of a single signal, at the center of an FFT bin from bearing $\pi / 8$, the threshold effect is observed for SNRs of $-50 \mathrm{~dB}$ or lower.

\section{Sinusoid with Bearing $\pi / 4$}

The sinusoid from bearing $\pi / 4$ represents the best possible signal condition. The bearing estimation performance is presented in Figure 4.21 The bearing estimation

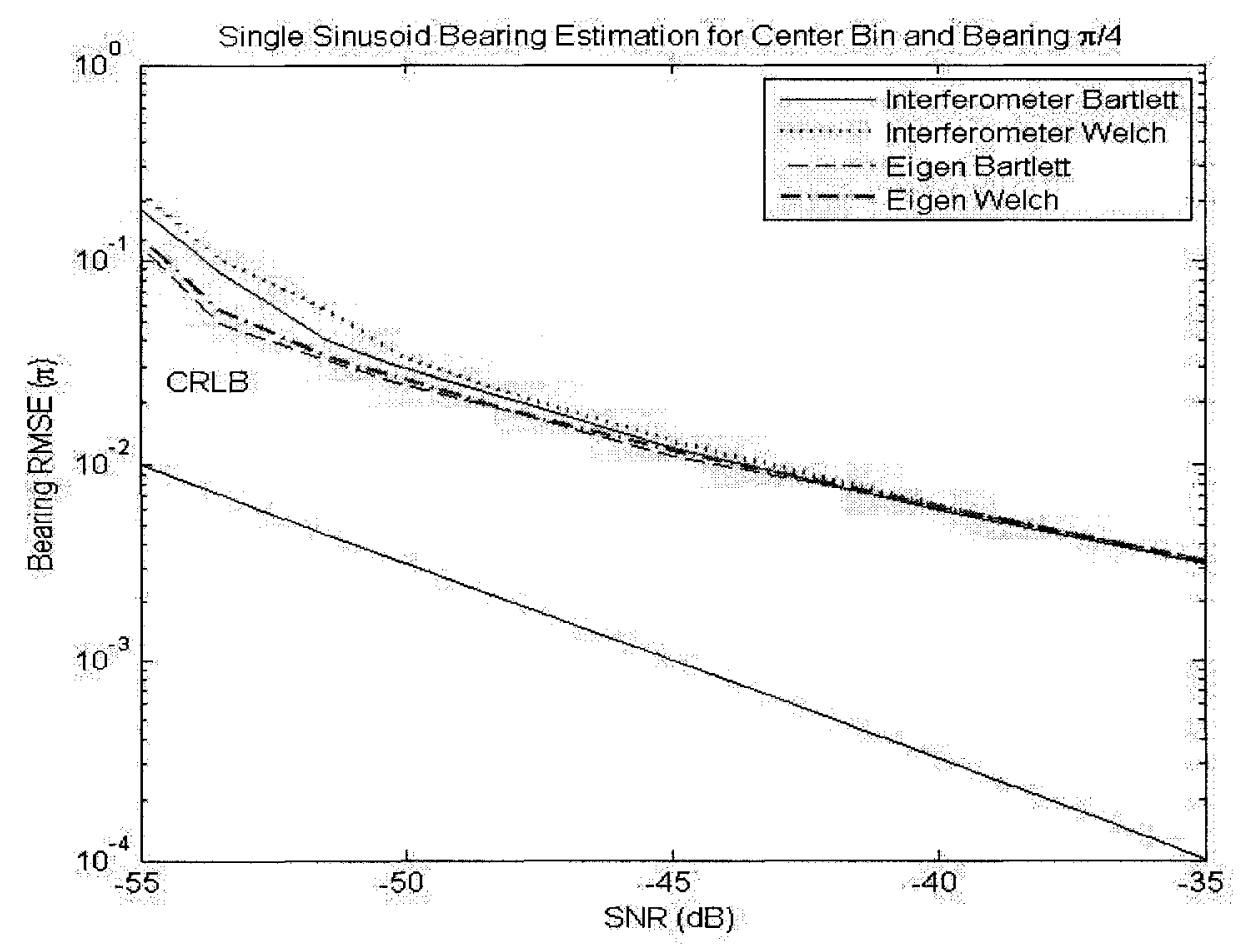

Figure 4.21: Bearing estimation performance for a single sinusoid at the center of an FFT bin, from bearing $\pi / 4$.

results indicate a slight performance benefit of the Bartlett method over the Welch method. Additionally, the Eigen-decomposition technique provides a slight increase 
in performance compared to the interferometer technique.

For the case of a single signal, at the center of an FFT bin from bearing $\pi / 4$. For the Bartlett method, the threshold effect is observed for SNRs of $-51 \mathrm{~dB}$ or lower. For the Welch method, the threshold effect is observed for SNRs of $-53 \mathrm{~dB}$ or lower.

\subsubsection{Single Sinusoid with Random Frequency}

The single sinusoid, random frequency simulations were performed by uniformly distributing the sinusoid frequency within frequency bin 8192 .

\section{Sinusoid with Bearing 0}

The random frequency results for a single sinusoid with bearing 0 are presented in Figure 4.22 .

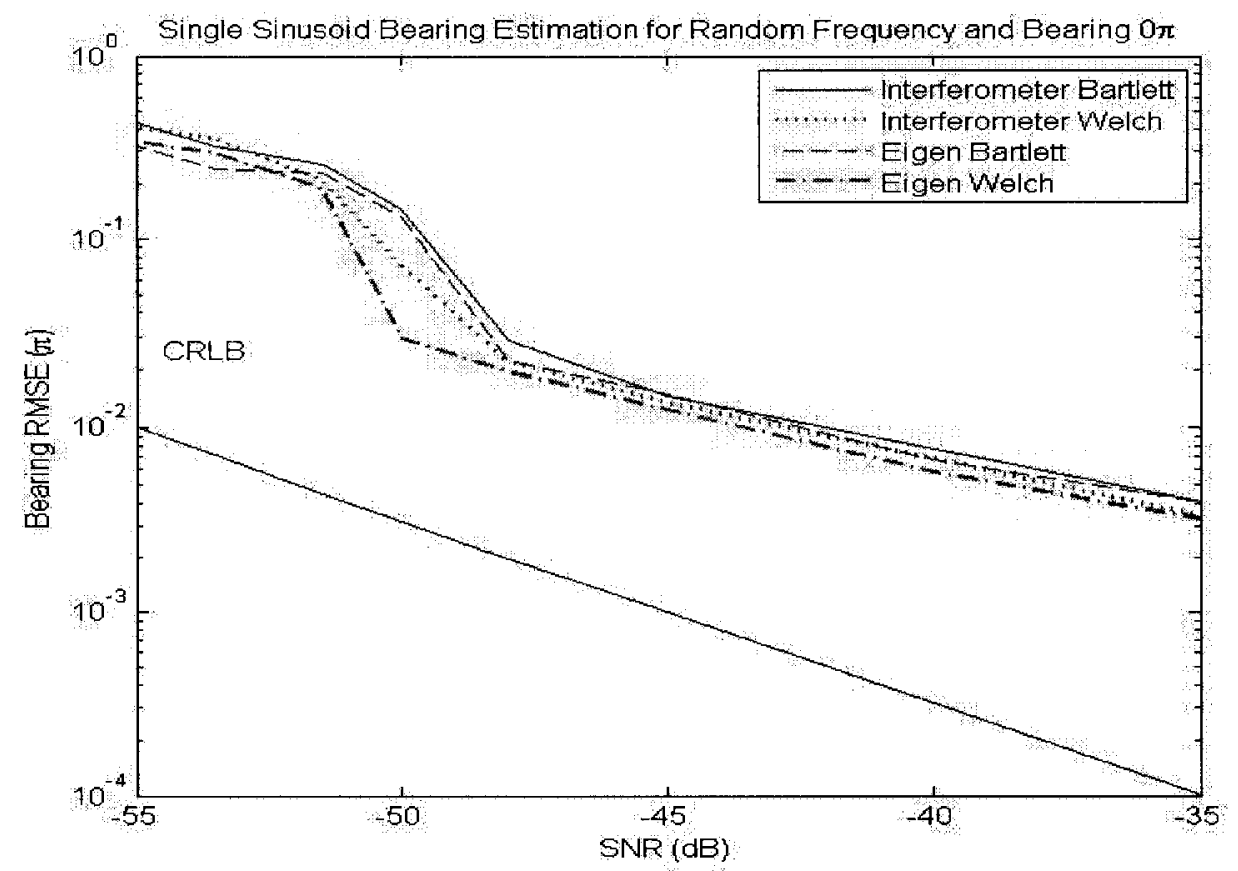

Figure 4.22: Bearing estimation performance for a single sinusoid with random frequency, from bearing 0 . 
As observed in the detection simulations, the Welch method outperforms the Bartlett method when the sinusoid frequency is random. Additionally, the Eigendecomposition technique demonstrated increased performance compared to the interferometer technique.

The threshold effect was observed for sinusoids with a SNR of $-48 \mathrm{~dB}$ or lower. An exception was the Eigen-decomposition / Welch method, where the threshold effect was observed for sinusoids of $-50 \mathrm{~dB}$ SNR or lower.

\section{Sinusoid with Bearing $\pi / 8$}

The random frequency results for a single sinusoid with bearing $\pi / 8$ are presented in Figure 4.23. For the case of a single sinusoid with random frequency and bear-

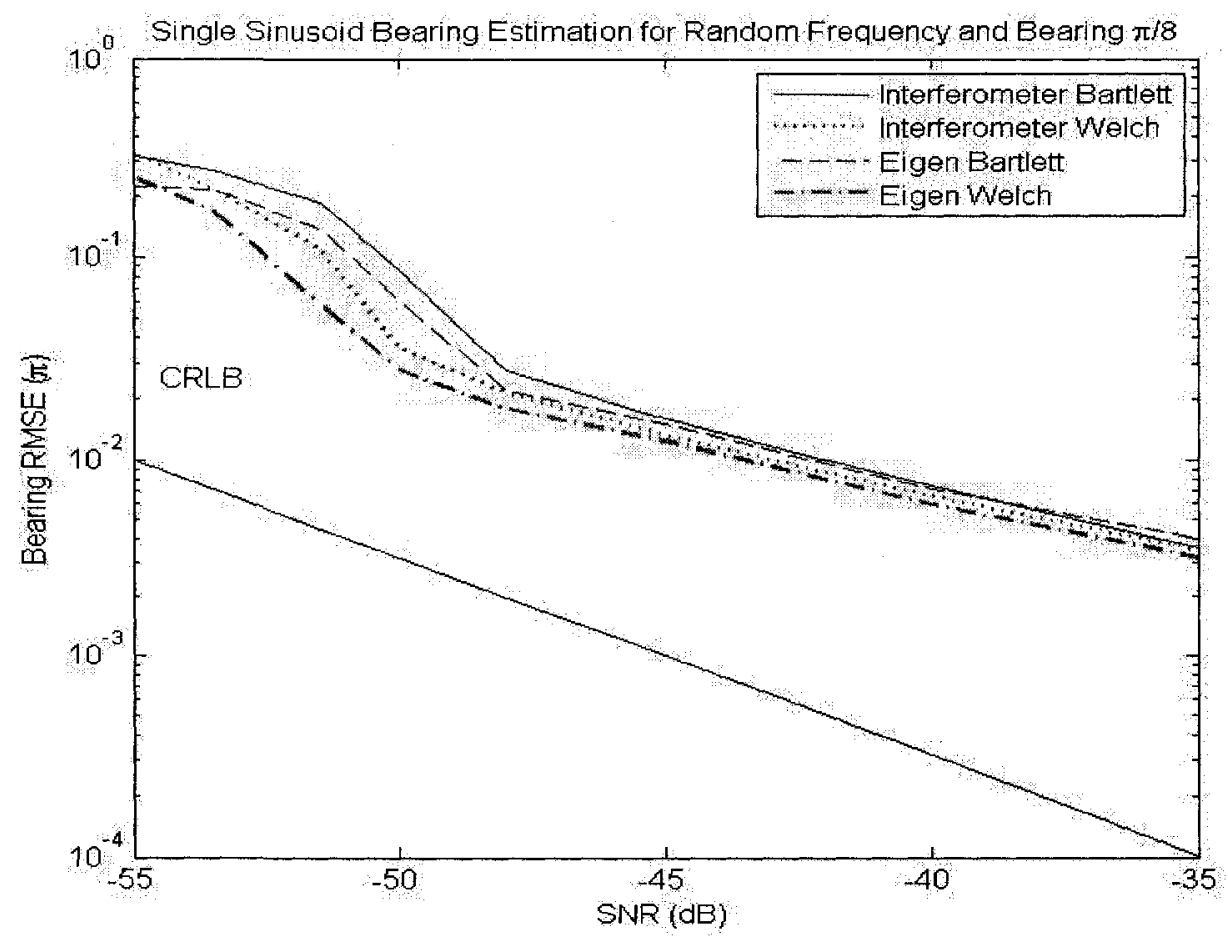

Figure 4.23: Bearing estimation performance for a single sinusoid with random frequency, from bearing $\pi / 8$. 
ing $\pi / 8$, the Welch method provides superior performance. Additionally, the Eigendecomposition technique provides a notable performance increase at lower SNRs.

For the interferometer technique, the threshold effect is observed for SNRs of -48 $\mathrm{dB}$ or lower, while the Eigen-decomposition technique shows a threshold effect for sinusoids with SNRs of $-50 \mathrm{~dB}$ or lower.

\section{Sinusoid with Bearing $\pi / 4$}

The random frequency results for a single sinusoid with bearing $\pi / 4$ are presented in Figure 4.24. For the case of a single sinusoid with random frequency and bear-

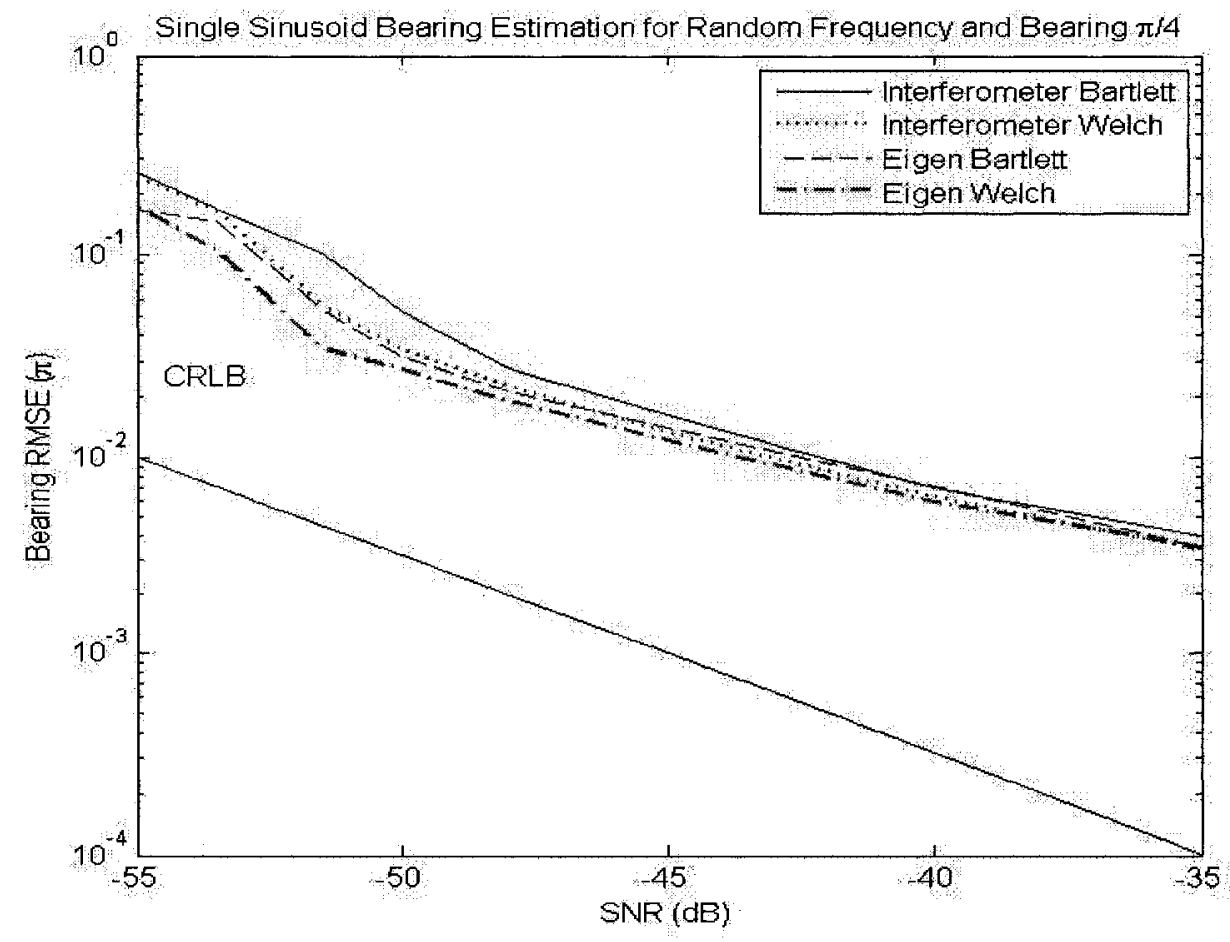

Figure 4.24: Bearing estimation performance for a single sinusoid with random frequency, from bearing $\pi / 4$.

ing $\pi / 4$, the Welch method provides superior performance. Additionally, the Eigendecomposition technique provides a notable performance increase at lower SNRs. 
For the Bartlett / interferometer technique, the threshold effect is observed for SNRs of $-48 \mathrm{~dB}$ or lower. For the Welch / Eigen-decomposition technique and Bartlett / Eigen-decomposition technique, the threshold effect is observed for sinusoids with SNRs of $-50 \mathrm{~dB}$ or lower. The best performance is observed for the Welch / Eigendecomposition technique where the threshold effect is observed for SNRs of $-52 \mathrm{~dB}$ or lower.

\subsubsection{Multiple Sinusoids with Random Frequency}

The intended application requires bearing estimation in the presence of multiple sinusoidal signals with a minimum separation of $12.5 \mathrm{kHz}$ or $81.92 \mathrm{FFT}$ bins. The simulations performed in this section employed 10 sinusoids. All sinusoids were assigned a random frequency and phase. The sinusoid of interest was assigned the desired bearing and SNR. The remaining sinusoids were assigned a uniformly distributed bearing from 0 to $2 \pi$ and a uniformly distributed SNR from -35 to $-45 \mathrm{~dB}$.

\section{Sinusoid with Bearing 0}

The random frequency results for multiple sinusoids with and a target sinusoid with bearing 0 are presented in Figure 4.25. 


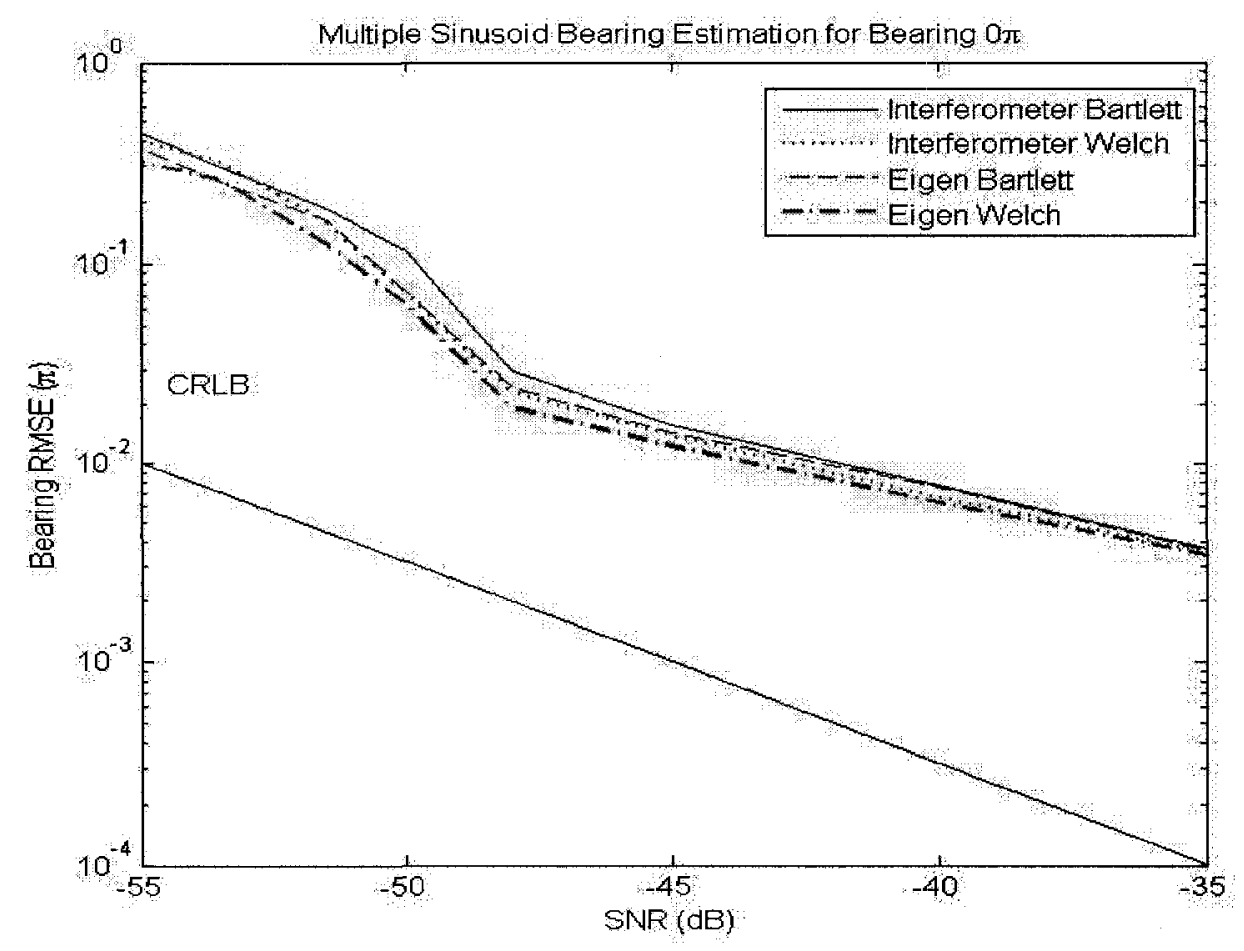

Figure 4.25: Bearing estimation performance for multiple sinusoids with random frequency, from bearing 0 .

Similar to the single sinusoid results, the Welch method slightly outperforms the Bartlett method and the Eigen-decomposition technique slightly outperforms the interferometer technique.

For all bearing estimation techniques, the threshold effect is observed for SNRs of $-48 \mathrm{~dB}$ or lower. 


\section{Sinusoid with Bearing $\pi / 8$}

The random frequency results for multiple sinusoids with and a target sinusoid with bearing $\pi / 8$ are presented in Figure 4.26.

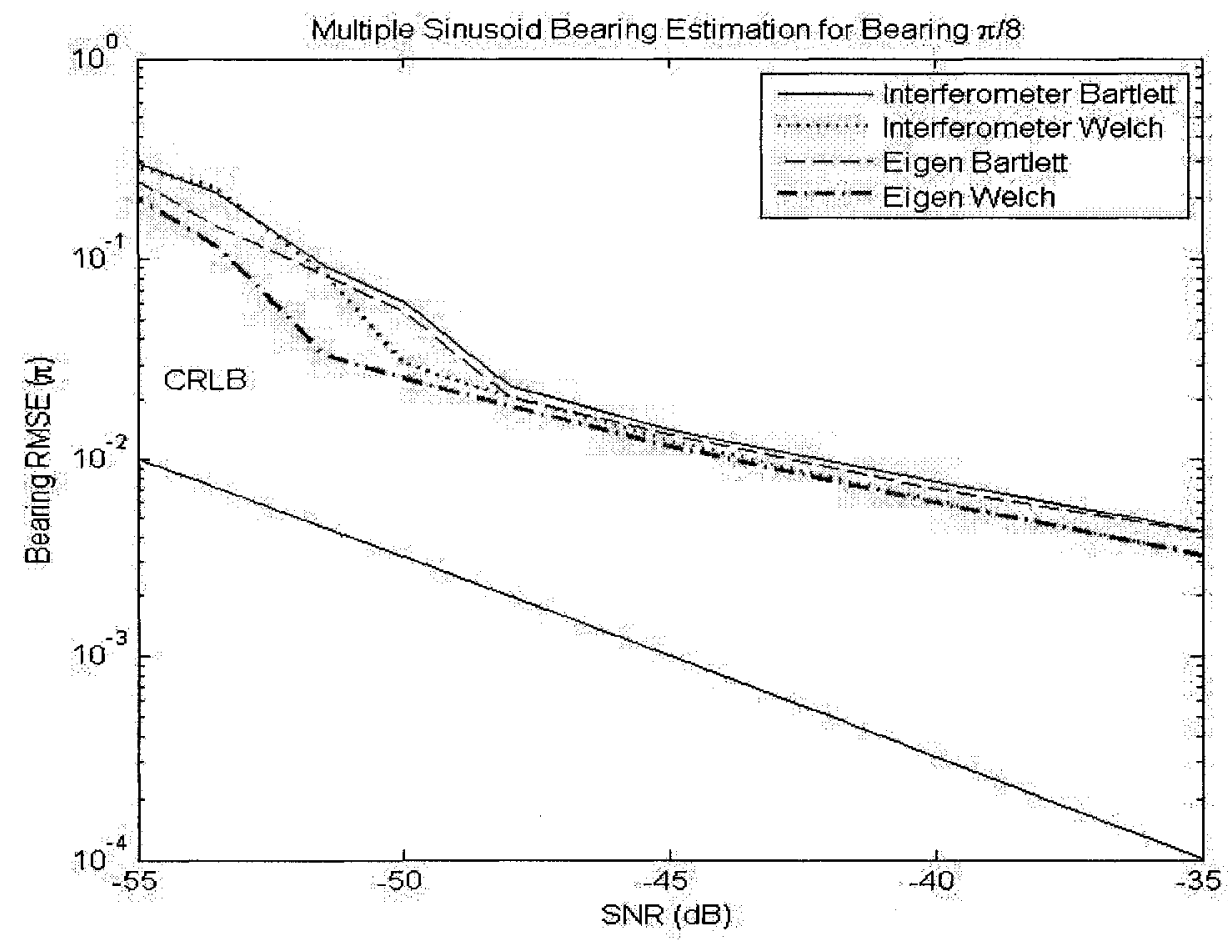

Figure 4.26: Bearing estimation performance for multiple sinusoids with random frequency, from bearing $\pi / 8$.

In the high SNR region, the performance of the two Bartlett methods appear slightly worse than the Welch method. Additionally, there appears to be little performance difference between the interferometer and Eigen-decomposition techniques in this region.

In the low SNR region, both Bartlett techniques exhibit a threshold effect for SNRs of $-48 \mathrm{~dB}$ or lower. The Welch / interferometer technique indicates a threshold effect at $-50 \mathrm{~dB}$ and lower, and the Welch / Eigen-decomposition technique experiences the threshold effect at SNRs of $-52 \mathrm{~dB}$ and lower. 


\section{Sinusoid with Bearing $\pi / 4$}

The random frequency results for multiple sinusoids with and a target sinusoid with bearing $\pi / 4$ are presented in Figure 4.27.

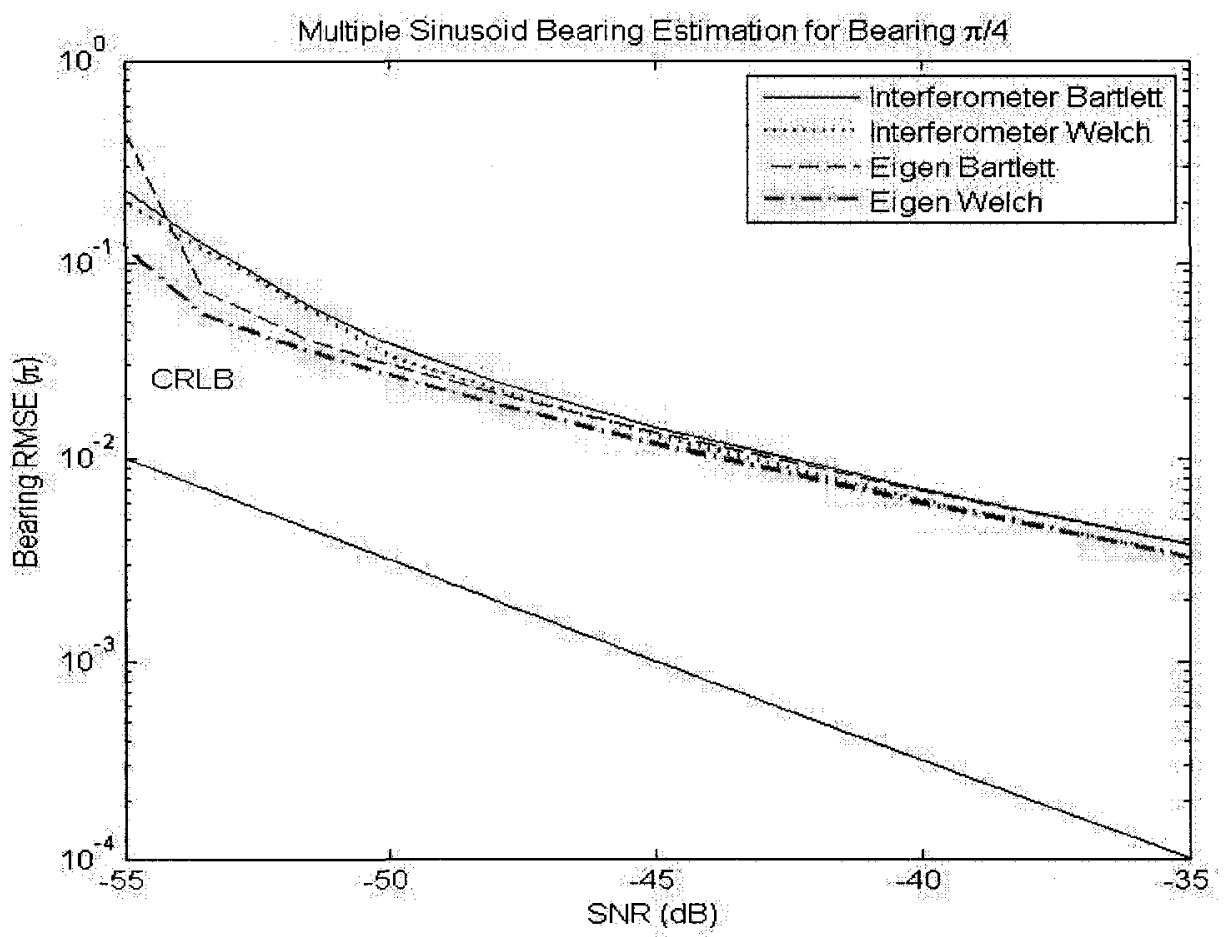

Figure 4.27: Bearing estimation performance for multiple sinusoids with random frequency, from bearing $\pi / 4$.

For the case of multiple sinusoids and a target sinusoid with bearing $\pi / 4$, the two Bartlett methods and two Welch methods exhibit similar bearing estimation performance.

The threshold effect is observed for the Bartlett methods at SNRs of $-50 \mathrm{~dB}$ or lower. The threshold effect for the Welch methods is observed for SNRs of $-53 \mathrm{~dB}$ and lower. The threshold effect appears to be more gradual for a target bearing of $\pi / 4$. 


\subsubsection{Effect of Sinusoid Separation on Bearing Estimation Error}

The digital signal processing architecture evaluated in this thesis utilizes a 32768 point FFT. Therefore, the input signal's minimum separation $(12.5 \mathrm{kHz})$ is much greater than four FFT bins (81.92 bins). In the previous subsections, it was shown that sufficient separation ensured single sinusoid bearing estimation performance, for the multiple sinusoid problem.

The large FFT size was selected to provide sufficient periodogram averaging, for one second of input data. However, if the minimum sinusoid separation is reduced, or interference signals are present, the single sinusoid performance assumption longer holds. It is important to understand the relationship between sinusoid separation and the bearing estimation accuracy.

To evaluate the effect of sinusoid separation on bearing estimation accuracy, a two tone scenario was evaluated for the 32768 point FFT case. Both tones had a SNR of $-45 \mathrm{~dB}$ and random phase. The signal of interest had a randomized frequency within frequency bin 8192 and a bearing of $\pi / 4$. The interfering signal had a random bearing and the frequency was randomized within the frequency bin at the specified frequency offset. The bearing estimation error for various FFT bin offsets is presented in Figure 4.28 .

As illustrated in Figure 4.28, the Welch method outperforms the Bartlett method for both the interferometer and the Eigen-decomposition, when the minimum separation is two FFT bins or greater. This performance difference can be attributed to the superior sidelobe suppression of the Hann window and increased averaging of the Welch method.

For the same signal conditions and averaging technique, the Eigen-decomposition 


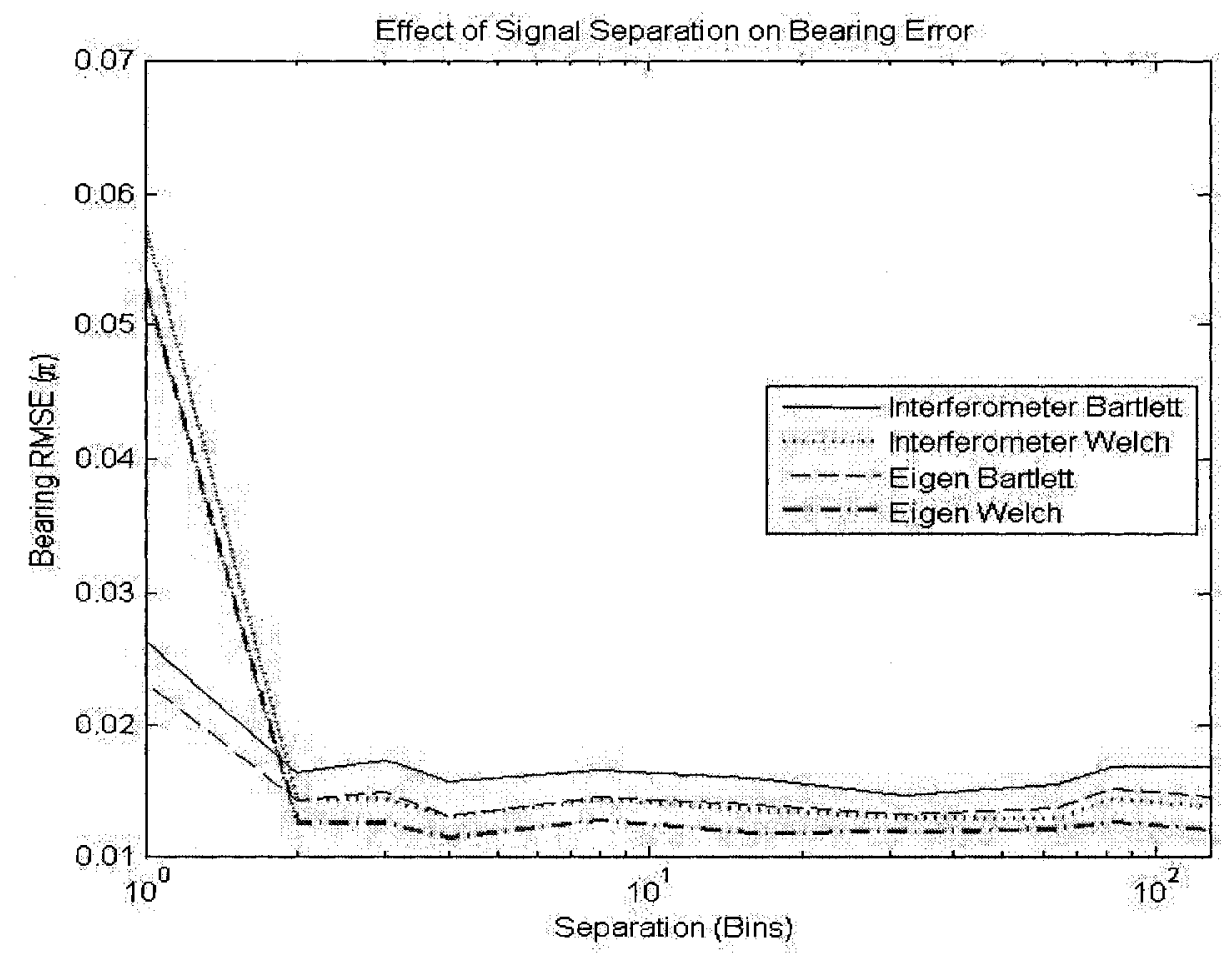

Figure 4.28: Effect of minimum sinusoid separation on bearing estimation error.

exceeds the performance of the interferometer. The performance difference was observed in previous sections and can be attributed to the interferometer only using a fraction of the multichannel PSD matrix.

For the case of two tones separated by one FFT bin, the Welch method exhibits a sharp rise in bearing estimation error. The increase in bearing error is due to the larger main lobe width of the Hann window ( $3 \mathrm{~dB}$ bandwidth of $2.00 \mathrm{bins}$ ) in comparison with the rectangular window ( $3 \mathrm{~dB}$ bandwidth of 1.21 bins).

\subsubsection{Conclusions}

When the sinusoid is centered on a FFT bin, the Bartlett method provides superior bearing estimation performance. 
When the frequency of the sinusoid is random, the Welch method outperforms the Bartlett method. The performance differences can be attributed to the window functions associated with the two spectral averaging techniques.

The bearing estimation performance was evaluated for the case of multiple sinusoids. The multiple sinusoid bearing estimation performance was comparable to the single sinusoid bearing estimation results.

For all simulations, the Eigen-decomposition technique was equivalent or better than the performance of the interferometer. However, the difference in performance was not substantial.

Threshold effects were observed below $-48 \mathrm{~dB}$ SNR, where a rapid increase in Root Mean Square (RMS) bearing error was observed.

If a less capable signal processing platform is used, the interferometer is considerably more efficient, with only a slight reduction in bearing estimation accuracy. However, if sufficient computational resources are available, the Eigen-decomposition technique should be used. A small increase in bearing estimation error will result in much larger errors in the triangulation of a target signal; since a position fix is generated from multiple bearing estimates and/or multiple bearing estimation platforms $[20]$. 


\section{Chapter 5}

\section{Conclusions and Future Work}

\subsection{Conclusions}

The signals studied in this thesis are multiple weak sinusoids with unknown amplitude, frequency, phase and bearing. A maximum of 10 signals are present, within the frequency band 55-60 MHz. Additionally, the minimum frequency separation of the sinusoids is $12.5 \mathrm{kHz}$. The sinusoid parameters are assumed to be constant for the duration of the observation interval.

The maximum likelihood receiver for sinusoids of unknown amplitude, frequency and phase was derived and shown to be the periodogram. Additionally, when the periodogram is implemented such that the minimum frequency separation is four FFT bins or greater, single sinusoid detection and bearing estimation performance bounds can be applied to the multiple sinusoid case.

A 32768 point FFT was required to provide sufficient signal processing gain, enabling detection and bearing estimation for the sinusoids. Using this configuration, the minimum signal separation was $81.92 \mathrm{FFT}$ bins. This frequency separation greatly exceeded the required minimum separation of four FFT bins. 
The data recorded in the one second observation period was segmented and averaged using both the Bartlett and Welch methods of spectral averaging. Both methods greatly reduced the variance of the periodogram estimates, enabling sinusoid detection and bearing estimation.

The sinusoid detection performance was in good agreement with the theoretical performance bounds for a sinusoid in AWGN. When the sinusoid is centered on an FFT bin, the Bartlett method provided superior performance compared to the Welch method. When the frequency of the sinusoid was random, significant performance degradation was observed for the Bartlett method, while only minimal performance degradation was observed for the Welch method. The detection performance of the Welch method exceeds the performance of the Bartlett method when the frequency is random; therefore, the Welch method is recommended for the signal processing problem studied in this thesis. Additionally, the 32768 point FFT provided sufficient frequency separation, permitting single sinusoid performance bounds to be used for the multiple sinusoid scenario.

The sinusoid bearing estimation was simulated for the four channel interferometer and Eigen-decomposition techniques, in conjunction with the Bartlett and Welch methods. Similar to the detection results, the bearing estimation performance, for a sinusoid centered on an FFT bin was better for the Bartlett method. When the frequency was randomized, the Welch method provided greater bearing estimation performance. In general, the Eigen-decomposition technique provides slighter better bearing accuracy than the interferometer. Threshold effects were observed with all bearing estimation techniques, resulting in a sharp increase in bearing error. The threshold effects were observed for SNRs of $-48 \mathrm{~dB}$ or lower. The threshold effect is experienced at higher SNR values for bearings from "end fire" conditions. The 
combination of Eigen-decomposition and Welch method exhibited the greatest performance and lowest threshold threshold effect, which is of great value when analyzing very weak signals. The effect of minimum frequency separation on bearing error was simulated and performance degradation was observed only when the signal separation was less than two FFT bins.

\subsection{Future Work}

\subsubsection{Algorithm Implementation}

The work presented in this thesis is simulation based. The next step in the development process is the implementation of the algorithm on a digital signal processing platform. Due to the computational demands of the signal detection and bearing estimation algorithms, the Xilinx Virtex-5 FPGA [25] is being investigated as the key processing element for this application.

\subsubsection{Minimum Sinusoid Separation}

The effect of minimum sinusoid separation on bearing estimation was simulated for two sinusoids of equal amplitude. The simulation results indicated a minimum separation of two FFT bins provided sufficient separation. Further analysis should be performed for sinusoids of unequal amplitude, possibly justifying the minimum separation of four FFT bins.

The simulations for minimum sinusoid separation should also be performed for sinusoid detection. 


\subsubsection{Channelization to Increase Bearing Estimation Accu- racy}

The signal processing strategy studied in this thesis utilized a single multichannel PSD matrix to calculate a sinusoid detection and bearing estimate. The multichannel PSD matrix was created while the data was recorded. Therefore, only blocks of input data were recorded instead of the entire observation period. This stategy was used to minimize the storage requirements of the signal processing system.

Unfortunately, this strategy requires the detection and bearing estimate to be generated from the same multichannel PSD matrix, limiting the bearing estimation performance. By separating the input data to the detection and bearing estimation, channelization in the bearing estimation block can be performed, resulting in greater bearing estimation accuracy. The channelization of the input data can be implemented using a Digital Downconverter (DDC). The center frequency of the DDC is provided to the bearing estimation processing block by the output of the detection processing block.

The DDC reduces the bandwidth of the input signal, resulting in less noise and a higher SNR. The higher SNR of the channelized input signal will provide greater bearing estimation accuracy using the same input signal.

The channelization can be implemented by recording an entire second of data for the four receiver channels and processing the detection and bearing estimation in series. This strategy will require increased storage and signal processing requirements.

Alternatively, if the response time is not critical, the channelization can be implemented by performing the signal detection on the first observation period and performing the bearing estimation on the second observation period. The memory requirements are less demanding for this strategy, while the signal processing require- 
ments are of similar complexity.

\subsubsection{Fast Orthogonal Search}

The FFT is the MLE estimator for a signal with unknown amplitude, frequency and phase. However, after completing the periodogram operation, an estimate of the amplitude and frequency of each detected sinusoid is available.

Using the results of the periodogram calculation, a secondary signal processing stage can be implemented providing higher resolution in the regions identified by the periodogram. A method of implementing higher resolution spectral estimation is the Fast Orthogonal Search (FOS) [27]. FOS provides increased spectral resolution with reduced computational requirements in comparison with high resolution techniques such as MUSIC. The performance of the FOS technique is dependant upon an initial prediction of the sinusoid frequencies detected. This information is provided by the primary periodogram signal processing stage.

The potential benefit of this strategy is a smaller multichannel PSD matrix required for the detection and bearing estimation processing blocks. Additionally, the higher resolution of the FOS technique should enable greater bearing estimation accuracy. The drawback for this strategy is increased signal processing delays and computational requirements due to the secondary spectral estimation block.

\subsubsection{Three Antenna Element Array}

The antenna configuration investigated in this report was a four channel circular array. Due to physical limitations of several mobile platforms, smaller antenna arrays must be considered.

Under these physical restrictions, a three antenna circular array may be consid- 
ered. This configuration will continue to provide 360 degrees of detection and bearing estimation. However, a reduction in detection and bearing estimation performance will result. An analysis, similar to the analysis in this thesis, should be performed to understand the performance degradation resulting from this design change.

The three element antenna array will require less physical area and reduced signal processing/power requirements. For these reasons, it is of greater interest to aerial platform applications.

\subsubsection{The Conjugate Gradient Method}

The Eigen-decomposition technique implemented in this thesis utilized the steepest descent algorithm to determine the local minimum of the cost function. This technique uses the first derivative of the cost function, making it a relative optimal method searching for the local minimum. Unfortunately, this technique requires many iterations when the cost function has a long and gradual slope to the local minimum.

The cost function for the bearing estimate was observed to have a relatively gradual slope. An alternative algorithm within the class of search techniques using the first derivative is the conjugate gradient method. The conjugate gradient method provides similar performance to the steepest descent algorithm. Additionally it requires fewer iterations when the valley structure of the cost function is long and gradual.

An analysis of the conjugate gradient method should be performed for the observed cost function to determine its impact on the bearing estimation signal processing and performance. 


\section{Bibliography}

[1] S. M. Kay, Fundamentals of Statistical Signal Processing: Detection Theory, ser. Prentice-Hall signal processing series, A. V. Oppenhheim, Ed. Prentice-Hall PTR, 1998, vol. 2.

[2] D. C. Rife, "Digital tone parameter estimation in the presence of gaussian noise," Engineering, Electrical, Polytechnic Institute of Brooklyn, 1973.

[3] H. C. So, Y. T. Chan, Q. Ma, and P. C. Ching, "Comparisson of various periodograms for sinusoid detection and frequency estimation," IEEE Transaction on Aerospace and Electrical Systems, vol. 35, no. 3, pp. 945-950, July 1999.

[4] G. S. Edelson and D. W. Tufts, "On the ability to estimate narrow-band signal parameters using towed arrays," IEEE Journal of Oceanic Engineering, vol. 17, no. 1, pp. 48-60, January 1992.

[5] L. P. Siedman, "Bearing estimation error with a linear array," IEEE Transactions on Audio and Electroacoustics, vol. 19, no. 2, pp. 147-157, June 1971.

[6] B. Wild and K. Ramchandran, "Detecting primary receivers for cognitive radio applications," First IEEE International Symposium on New Frontiers in Dynamic Spectrum Access Networks, pp. 124-130, November 2005. 
[7] S. M. Kay, "Accurate frequency estimation at low signal-to noise ratio," IEEE Transactions on Acoustics, Speech and Signal Processing, vol. 32, no. 3, pp. 540547, June 1984.

[8] D. C. Rife and R. R. Boorstyn, "Multiple tone parameter estimation from discrete-time observations," The Bell System Technical Journal, vol. 55, no. 9, pp. 1389-1410, November 1976.

[9] S. Holme, "Optimum fft-based frequency acquisition with applications to cospassarsat," IEEE Transactions on Aerospace and Electrical Systems, vol. 29, no. 2, pp. 464-475, April 1993.

[10] D. C. Rife and R. R. Boorstyn, "Single-tone parameter estimation from discretetime observations," IEEE Transations on Information Theory, vol. 20, no. 5, pp. 591-598, September 1974.

[11] S. M. Kay, Fundamentals of Statistical Signal Processing: Estimation Theory, ser. Prentice-Hall signal processing series, A. V. Oppenhheim, Ed. PrenticeHall PTR, 1993, vol. 1.

[12] A. D. Whalen, Detection of Signals in Noise, ser. Electrical Science Series. New York, USA: Academic Press Inc., 1971.

[13] L. C. Palmer, "Course frequency estimation using the discrete fourier transform," IEEE Transactions on Information Theory, vol. 20, no. 1, pp. 104-109, January 1974.

[14] J. G. Proakis, Digital Communications, 4th ed. New York, USA: McGraw Hill, 2001. 
[15] S. L. J. Marple, Digital Spectral Analysis with Applications, ser. Prentice Hall Signal Processing Series. New Jersey, USA: Prentice-Hall Inc., 1987.

[16] P. Stoica and A. Nehorai, "Music, maximum likelihood, and cramer-rao bound," IEEE Transactions on Acoustics, Speech and Signal Processing, vol. 37, no. 5, pp. 720-741, May 1989.

[17] H. R. Karimi and A. Manikas, "Manifold of a planar array and its effects on the accuracy of direction-finding systems," IEE Proceedings Radar, Sonar and Navigation, vol. 143, no. 6, pp. 349-357, December 1996.

[18] H. L. Van Trees, Optimum Array Signal Processing, ser. Detection, Estimation and Modulation Theory. John Wiley and Sons, Inc., 2002, vol. 4.

[19] V. K. Madisetti and D. B. Williams, The Digital Signal Processing Handbook, ser. Electrical Engineering Handbook. CRC Press, 1997, vol. 11.

[20] R. A. Poisel, Introduction to Communications Electronic Warfare Systems. Norwood, USA: Artech House Inc., 2002.

[21] E. Kreyszig, Advanced Engineering Mathmatics, 7th ed. John Wiley \& Sons, Inc, 1993.

[22] W. K. Nicholson, Linear Algebra with applications, 3rd ed. PWS Publishing Company, 1995.

[23] W. H. Press, B. P. Flannery, S. A. Teukolsky, and W. T. Vetterling, Numerical Recipes In C. Cambridge University Press, 1990.

[24] Fast Fourier Transform v5.0 Product Specification, Xilinx Inc, October 2007.

[25] Virtex-5 Family Overview, 4th ed., Xilinx Inc., April 2008. 
[26] F. J. Harris, "On the use of windows for harmonic analysis with the discrete fourier transform," Proceedings of the IEEE, vol. 6, no. 1, pp. 51-83, January 1978.

[27] K. M. Adeney and M. J. Korenberg, "Fast orthogonal search for array processing and spectral estimation," IEE Proceedings - Vision, Image and Signal Processing, vol. 141, no. 1, pp. 13-18, February 1994.

[28] M. Barkat, Signal Detection and Estimation, 2nd ed. Norwood, USA: Artech House Inc., 2005.

[29] B. G. Quinn and E. J. Hannan, The Estimation and Tracking of Frequency, ser. Cambridge Series in Statistical and Probabilistic Mathematis. Cambridge, UK: Cambridge University Press, 2001.

[30] R. A. Poisel, Target Acquisition in Communications Electronic Warfare Systems. Norwood, USA: Artech House Inc., 2004.

[31] W. A. Gardner, Statistical Spectral Analysis, ser. Prentice Hall Information and System Sciences Series. New Jersey, USA: Prentice-Hall Inc., 1988.

[32] A. Grigorakis, "Application of detection theory to the measurement of the minimum detectable signal for a sinusoid in gaussian noise displayed on a lofagram," Defence Science and Technology Organisation, Technical Report DSTO-TR0568, August 1997.

[33] J. Tsui, Digital Techniques for Wideband Receivers, 2nd ed. Norwood, USA: Artech House Inc., 2001. 
[34] A. V. Oppenheim and R. W. Shafer, Discrete-Time Signal Processing, 2nd ed., ser. Prentice Hall Signal Processing Series. New Jersey, USA: Prentice-Hall Inc., 1999.

[35] A. Papoulis, Probability, Random VAriables and Stochastic Processes, ser. McGraw-Hill Series in Systems Science. New York, USA: McGraw-Hill, 1965.

[36] J. R. Ogden and M. Fogiel, Handbook of Mathematical, Scientific, and Engineering: Formulas, Tables, Functions, Graphs, Transforms. Research and Education Association, 1984. 


\section{Appendix A}

\section{Probability and Random Process}

\section{Theory}

The following section defines several variables and concepts used in this report. It also provides a basic review of probability and random process theory, expanded upon in further sections.

\section{A.1 Deterministic vs. Stochastic Processes}

\section{A.1.1 Deterministic Process}

A function or signal is deterministic if the future value can be predicted when the current value is known. The cosine and sine functions are examples of deterministic processes. 


\section{A.1.2 Stochastic Process}

A function is stochastic if its future value can only be described statistically, regardless of the current knowledge of the signal. Most communication signals are considered stochastic.

\section{A.2 Stochastic Process Properties}

We define a random variable $\mathbf{x}$ as a variable whose values are random, but whose statistical distribution is known. The distribution of $\mathbf{x}$ is governed by the probability distribution function $\mathrm{F}(x)$, defined $\mathrm{F}(x)=\operatorname{Pr}(\mathbf{x} \leq x)$, indicating the probability that the random variable $\mathbf{x}$ is less than or equal to $x$. The probability density function $\mathrm{p}(x)$, is the derivative of $\mathrm{F}(x)$ defined $\mathrm{p}(x)=\frac{d F(x)}{d x}$.

The expectation of the random variable $\mathbf{x}$, denoted $\mathrm{E}\{\mathbf{x}\}$ is given by:

$$
E\{\mathbf{x}\}=\int_{-\infty}^{\infty} x p(x) d x=m_{x}
$$

The expectation of $\mathbf{x}$ is also referred to as the mean of $\mathbf{x}$ or the first moment of $\mathbf{x}[14]$.

The expectation of the squared magnitude of the random variable $\mathbf{x}$, is given by:

$$
E\left\{|\mathbf{x}|^{2}\right\}=\int_{-\infty}^{\infty}|x|^{2} p(x) d x
$$

The expectation of the squared magnitude of $\mathbf{x}$ is also referred to as the meansquared value or the second moment of $\mathbf{x}$.

The variance, denoted $\sigma^{2}$, is a measure of the mean-squared deviation of $\mathbf{x}$ from 
its mean. The variance is given by:

$$
\sigma^{2}=\int_{-\infty}^{\infty}|x-E\{\mathbf{x}\}|^{2} p(x) d x=E\left\{|\mathbf{x}|^{2}\right\}-|E\{\mathbf{x}\}|^{2}
$$

In the case of two random variables, $\mathrm{x}_{1}$ and $\mathbf{x}_{2}$, with joint $\mathrm{pdf} \mathrm{p}\left(\mathrm{x}_{1}, \mathrm{x}_{2}\right)$, the joint moment is given by:

$$
E\left\{\mathbf{x}_{1}^{k} \mathbf{x}_{2}^{n}\right\}=\int_{-\infty}^{\infty} \int_{-\infty}^{\infty} x_{1}^{k} x_{2}^{n} p\left(x_{1}, x_{2}\right) d x_{1} d x_{2}
$$

and the joint central moment is given by:

$$
E\left\{\left(\mathbf{x}_{1}-m_{x_{1}}\right)^{k}\left(\mathbf{x}_{2}-m_{x_{2}}\right)^{n}\right\}=\int_{-\infty}^{\infty} \int_{-\infty}^{\infty}\left(x_{1}-m_{x_{1}}\right)^{k}\left(x_{2}-m_{x_{2}}\right)^{n} p\left(x_{1}, x_{2}\right) d x_{1} d x_{2}
$$

The special case where $\mathrm{k}=\mathrm{n}=1$ is of special importance. Under this condition, the joint moment defines the correlation and the joint central moment defines the covariance of the random variables $\mathbf{x}_{1}$ and $\mathbf{x}_{2}$. The correlation between $\mathbf{x}_{1}$ and $\mathbf{x}_{2}$ is given by:

$$
R_{x_{1} x_{2}}=E\left\{\mathbf{x}_{1} \mathbf{x}_{2}\right\}=\int_{-\infty}^{\infty} \int_{-\infty}^{\infty} x_{1} x_{2} p\left(x_{1}, x_{2}\right) d x_{1} d x_{2}
$$

and the covariance is given by: 


$$
\begin{aligned}
C_{x_{1} x_{2}} & =E\left\{\left(\mathbf{x}_{1}-m_{x_{1}}\right)\left(\mathbf{x}_{2}-m_{x_{2}}\right)\right\} \\
& =\int_{-\infty}^{\infty} \int_{-\infty}^{\infty}\left(x_{1}-\mu_{x_{1}}\right)\left(x_{2}-\mu_{x_{2}}\right) p\left(x_{1}, x_{2}\right) d x_{1} d x_{2} \\
& =\int_{-\infty}^{\infty} \int_{-\infty}^{\infty} x_{1} x_{2} p\left(x_{1}, x_{2}\right) d x_{1} d x_{2}-m_{x_{1}} m_{x_{2}} \\
& =E\left\{\mathbf{x}_{1} \mathbf{x}_{2}\right\}-m_{x_{1}} m_{x_{2}}
\end{aligned}
$$

\section{A.2.1 The Central Limit Theorem}

We define $x_{n}$ as a sequence of $\mathrm{n}$ independent identically distributed (iid) random variables, with finite means and variances. Also, let $S_{n}$ be the sum of these variables and $\mathbf{z}_{n}$ be the normalized random variable defined:

$$
\mathbf{z}_{n}=\frac{S_{n}-E\left\{S_{n}\right\}}{\sigma_{S_{n}}}
$$

The central limit theorem states that as $n \rightarrow \infty$, the random variable $\mathbf{z}_{n}$ converges to a normally distributed random variable with zero mean and a standard deviation of one.

The normal distribution, also referred to as Gaussian distribution, is of particular importance to detection theory and signal processing. If the conditions of the central limit theorem a met, the distribution of noise source will approach that of a Gaussian distribution. 


\section{A.2.2 The Gaussian Distribution}

The Gaussian distribution, for a real random variable $\mathbf{x}$, defined over $(-\infty<\mathbf{x}<\infty)$ is given by [12] :

$$
p(\mathbf{x})=\frac{1}{\sqrt{(2 \pi) \sigma_{x}^{2}}} e^{-\frac{\left(x-m_{x}\right)^{2}}{2 \sigma_{x}^{2}}}
$$

Therefore, the normalized Gaussian random variable (z) has a probability density function:

$$
p(\mathbf{z})=\frac{1}{\sqrt{(2 \pi)}} e^{-\frac{z^{2}}{2}}
$$

The normalized Gaussian probability density function is illustrated in Figure A.1.

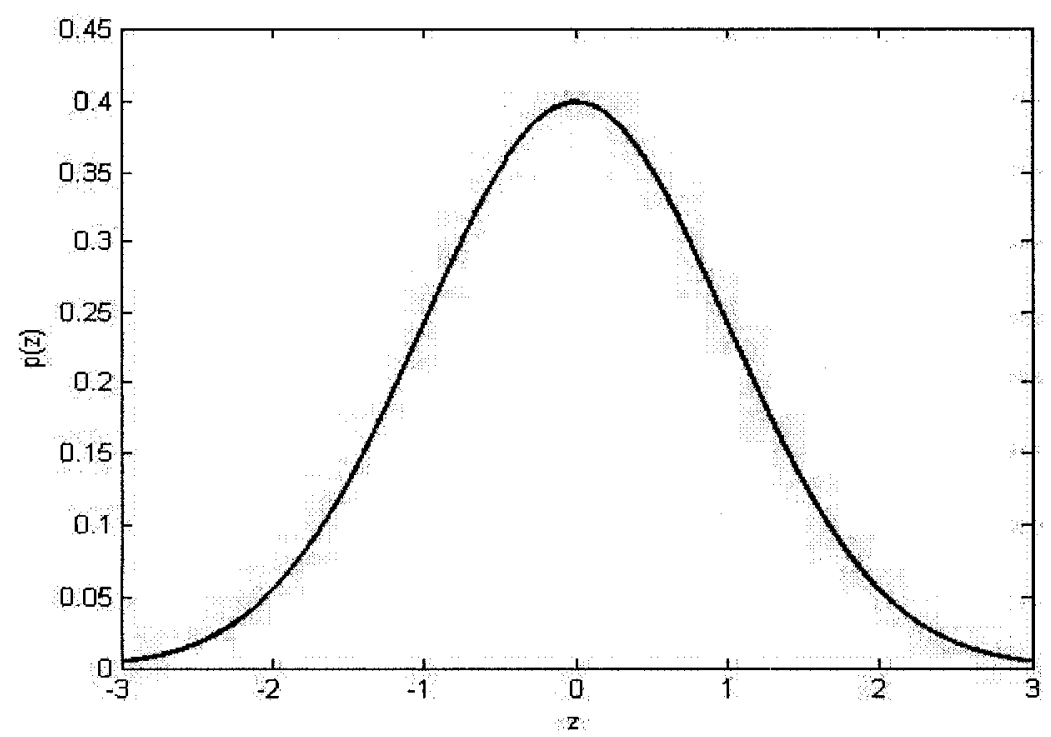

Figure A.1: The normalized Gaussian density function.

The multivariate Gaussian probability density function [15] for a vector of $\mathrm{N}$ real 
random variables $\mathbf{x}=\left[x_{1} x_{2} \ldots x_{N}\right]^{T}$ is given by:

$$
p(\mathbf{x})=\frac{1}{\sqrt{(2 \pi)^{N} \operatorname{det} \mathbf{C}_{N}}} e^{\left[-\frac{1}{2}\left(\mathbf{x}-m_{x}\right)^{T} \mathbf{C}_{N}^{-1}\left(\mathbf{x}-m_{x}\right)\right]}
$$

where $\mathbf{C}_{N}$ is the $\mathrm{N} \times \mathrm{N}$ covariance matrix defined:

$$
\begin{aligned}
\mathbf{C}_{N} & =E\left\{\left(\mathbf{x}-m_{x}\right)\left(\mathbf{x}-m_{x}\right)^{T}\right\} \\
= & \left(\begin{array}{cccc}
\sigma_{x_{1}}^{2} & \operatorname{cov}\left(x_{1} x_{2}\right) & \ldots & \operatorname{cov}\left(x_{1} x_{N}\right) \\
\operatorname{cov}\left(x_{2} x_{1}\right) & \sigma_{x_{2}}^{2} & \ldots & \operatorname{cov}\left(x_{2} x_{N}\right) \\
\vdots & \vdots & \ddots & \vdots \\
\operatorname{cov}\left(x_{N} x_{1}\right) & \operatorname{cov}\left(x_{N} x_{2}\right) & \ldots & \sigma_{x_{N}}^{2}
\end{array}\right)
\end{aligned}
$$

For the special case of N, i.i.d gaussian random variables, the variables are uncorrelated and the above equation simplifies to:

$$
p(\mathbf{x})=\frac{1}{\sqrt{(2 \pi)^{N}} \sigma_{x}^{N}} e^{\left[-\frac{1}{2 \sigma_{x}^{2}} \sum_{k=0}^{N-1}\left(x_{k}-m_{x}\right)^{2}\right]}
$$

\section{A.3 The Cramer-Rao Lower Bound and Estimator Performance}

The Cramer-Rao Lower Bound (CRLB) provides a lower bound on the variance of an unbiased estimator. The CRLB is useful in detection theory as it indicates the asymptotically optimum performance of a maximum likelihood estimator. 


\section{A.3.1 Characteristics of Estimator Performance}

An estimator is a random variable and may take on multiple values. Since the quality of different estimators may vary considerably, several terms have been defined to indicate the behavior and performance of an estimator [28].

The bias of an estimator $\hat{\alpha}$, for the true value $\alpha$ is defined as:

$$
b(\hat{\alpha})=\alpha-E\{\hat{\alpha}\}
$$

where $\mathrm{E}\{\cdot\}$ represents the expected value of $\alpha[14]$.

The estimator $\hat{\alpha}$ is called unbiased if $\alpha=E\{\hat{\alpha}\}$.

The estimator variance of $\hat{\alpha}$ is is given by:

$$
\operatorname{var}\{\hat{\alpha}\}=E\left\{\hat{\alpha}^{2}\right\}-E^{2}\{\hat{\alpha}\}
$$

An estimator is consistent if the estimate $\hat{\alpha}$ approaches the true value $\alpha$ in some probabilistic case (i.e. as the number of observations increases, the mean and variance approach zero [15]).

As stated in the previous section, the CRLB is a lower bound on the variance of an unbiased estimator. An estimator $\hat{\alpha}$ is called efficient if it achieves the CRLB.

\section{A.3.2 Scalar CRLB}

The CRLB is a lower bound on the variance of an unbiased statistical estimator, given by the inverse of the Fisher information [29], denoted $\mathcal{J}\{\alpha\}$. The Fisher information matrix identifies the amount of information a random variable contains with respect to an unknown parameter $\alpha$. The scalar CRLB is often used to indicate how well a 
single parameter of a signal (such as amplitude, frequency, phase or direction) can be estimated [30]. The CRLB, denoted var $\{\hat{\alpha}\}$, is given by:

$$
\operatorname{var}\{\hat{\alpha}\} \geq \frac{1}{\mathcal{J}\{\alpha\}}=\left(-E\left\{\frac{\partial^{2} \ln p(\mathbf{x} ; \alpha)}{\partial \alpha^{2}}\right\}\right)^{-1}
$$

where $\mathrm{p}(\mathbf{x} ; \alpha)$ is the probability density function of $\mathbf{x}$, with unknown parameter $\alpha$.

\section{A.3.3 Vector CRLB}

The vector CRLB analyzes the performance of an unbiased estimator for a vector $\alpha$ of unknown parameters [30]. The case of multiple known parameters is common in the study of signal detection and spectral estimation. In this case, $\hat{\alpha}$ is the estimate of $\alpha$, and $\alpha_{i}$ is a scalar representing its $i^{\text {th }}$ element, where $\mathrm{i}=1, \ldots, \mathrm{n}$. The CRLB for $\alpha_{i}$ is given by the diagonal elements of the inverse Fisher information matrix:

$$
\operatorname{var}\left\{\hat{\alpha}_{i}\right\} \geq \frac{1}{\mathbf{J}\{\alpha\}}=\left(-E_{p(z ; \alpha)}\left\{\frac{\partial^{2} \ln \mathbf{p}(\mathbf{z} ; \alpha)}{\partial \alpha^{2}}\right\}\right)^{-1}
$$

where $\mathbf{J}$ is an $n \times n$ matrix with elements:

$$
\mathbf{J}\left\{\alpha_{i j}\right\}=-E_{p(\mathbf{z} ; \alpha)}\left\{\frac{\partial^{2} \ln p(\mathbf{z} ; \alpha)}{\partial \alpha_{i} \partial \alpha j}\right\}
$$

Consider the case of a single complex sinusoid in Complex White Gaussian Noise (CWGN) with all parameters unknown $(\alpha=[\omega, \mathrm{A}, \phi])$. The probability density 
function is given by equation A.14, and its complex representation is given by:

$$
p(\mathbf{z} ; \alpha)=\left(\frac{1}{\pi \sigma_{z}^{2}}\right)^{N} e^{-\frac{1}{\sigma_{z}^{2}} \sum_{n=0}^{N-1}\left|y_{z}[n]-\mu_{z}[n]\right|^{2}}
$$

where $\mu_{z}[n]=A e^{j(\omega n+\phi)}$.

The elements of the Fisher information matrix are calculated as follows [11]:

$$
\begin{gathered}
\ln p(\mathbf{z} ; \alpha)=-\ln \left(\pi^{N} \sigma_{z}^{2 N}\right)-\frac{1}{\sigma_{z}^{2}} \sum_{n=0}^{N-1}\left|y_{z}[n]-\mu_{z}[n]\right|^{2} \\
\frac{\partial^{2} \ln p(\mathbf{z} ; \alpha)}{\partial \alpha_{i} \partial \alpha_{j}}=\frac{2}{\sigma_{z}^{2}} \operatorname{Re}\left\{\sum_{n=0}^{N-1}-\frac{\partial \mu_{z}^{*}[n]}{\partial \alpha_{i}} \frac{\partial \mu_{z}[n]}{\partial \alpha_{j}}\right\}
\end{gathered}
$$

Therefore, the coefficients of the Fisher information matrix are given by:

$$
\mathbf{J}\left\{\alpha_{i j}\right\}=-E\left\{\frac{\partial^{2} \ln p(z ; \alpha)}{\partial \alpha_{i} \partial \alpha_{j}}\right\}=\frac{2}{\sigma_{z}^{2}} \operatorname{Re}\left\{\sum_{n=0}^{N-1} \frac{\partial \mu_{z}^{*}[n] \partial \mu_{z}[n]}{\partial \alpha_{i} \partial \alpha_{j}}\right\}
$$

Substituting $\mu_{z}[n]$ into equation A.23 and evaluating the individual elements of the Fisher information matrix, we get:

$$
\begin{aligned}
\mathbf{J}\left\{\alpha_{11}\right\} & =\frac{2}{\sigma_{z}^{2}} \operatorname{Re}\left\{\sum_{n=0}^{N-1} \frac{\partial A e^{-j(\omega n+\phi)}}{\partial \omega} \frac{\partial A e^{j(\omega n+\phi)}}{\partial \omega}\right\} \\
& =\frac{2}{\sigma_{z}^{2}} \operatorname{Re}\left\{\sum_{n=0}^{N-1}(-j n) A e^{-j(\omega n+\phi)}(j n) A e^{j(\omega n+\phi)}\right\} \\
& =\frac{2 A^{2}}{\sigma_{z}^{2}} \sum_{n=0}^{N-1} n^{2}
\end{aligned}
$$




$$
\begin{aligned}
\mathbf{J}\left\{\alpha_{12}\right\} & =\frac{2}{\sigma_{z}^{2}} \operatorname{Re}\left\{\sum_{n=0}^{N-1} \frac{\partial A e^{-j(\omega n+\phi)}}{\partial \omega} \frac{\partial A e^{j(\omega n+\phi)}}{\partial A}\right\} \\
& =\frac{2}{\sigma_{z}^{2}} \operatorname{Re}\left\{\sum_{n=0}^{N-1}\left(-A j n e^{-j(\omega n+\phi)}\right)\left(e^{j(\omega n+\phi)}\right)\right\} \\
& =\frac{2}{\sigma_{z}^{2}} \operatorname{Re}\left\{\frac{-j A N(N-1)}{2}\right\} \\
& =0
\end{aligned}
$$

$$
\begin{aligned}
\mathbf{J}\left\{\alpha_{13}\right\} & =\frac{2}{\sigma_{z}^{2}} \operatorname{Re}\left\{\sum_{n=0}^{N-1} \frac{\partial A e^{-j(\omega n+\phi)}}{\partial \omega} \frac{\partial A e^{j(\omega n+\phi)}}{\partial \phi}\right\} \\
& =\frac{2}{\sigma_{z}^{2}} \operatorname{Re}\left\{\sum_{n=0}^{N-1}\left(-A j n e^{-j(\omega n+\phi)}\right)\left(A j e^{j(\omega n+\phi)}\right)\right\} \\
& =\frac{2 A^{2}}{\sigma_{z}^{2}} \sum_{n=0}^{N-1} n
\end{aligned}
$$

$$
\begin{aligned}
\mathbf{J}\left\{\alpha_{22}\right\} & =\frac{2}{\sigma_{z}^{2}} \operatorname{Re}\left\{\sum_{n=0}^{N-1} \frac{\partial A e^{-j(\omega n+\phi)}}{\partial A} \frac{\partial A e^{j(\omega n+\phi)}}{\partial A}\right\} \\
& =\frac{2}{\sigma_{z}^{2}} \operatorname{Re}\left\{\sum_{n=0}^{N-1}\left(e^{-j(\omega n+\phi)}\right)\left(e^{j(\omega n+\phi)}\right)\right\} \\
& =\frac{2 N}{\sigma_{z}^{2}}
\end{aligned}
$$

$$
\begin{aligned}
\mathbf{J}\left\{\alpha_{23}\right\} & =\frac{2}{\sigma_{z}^{2}} \operatorname{Re}\left\{\sum_{n=0}^{N-1} \frac{\partial A e^{-j(\omega n+\phi)}}{\partial A} \frac{\partial A e^{j(\omega n+\phi)}}{\partial \phi}\right\} \\
& =\frac{2}{\sigma_{z}^{2}} \operatorname{Re}\left\{\sum_{n=0}^{N-1}\left(e^{-j(\omega n+\phi)}\right)\left(A j e^{j(\omega n+\phi)}\right)\right\} \\
& =\frac{2}{\sigma_{z}^{2}} \operatorname{Re}\{j A N\} \\
& =0
\end{aligned}
$$




$$
\begin{aligned}
\mathbf{J}\left\{\alpha_{33}\right\} & =\frac{2}{\sigma_{z}^{2}} \operatorname{Re}\left\{\sum_{n=0}^{N-1} \frac{\partial A e^{-j(\omega n+\phi)}}{\partial \phi} \frac{\partial A e^{j(\omega n+\phi)}}{\partial \phi}\right\} \\
& =\frac{2}{\sigma_{z}^{2}} \operatorname{Re}\left\{\sum_{n=0}^{N-1}\left(-A j e^{j(\omega n+\phi)}\right)\left(A j e^{j(\omega n+\phi)}\right)\right\} \\
& =\frac{2 A^{2} N}{\sigma_{z}^{2}}
\end{aligned}
$$

Therefore, the Fisher information matrix for a single complex sinusoid is:

$$
\mathbf{J}\{\alpha\}=\frac{2}{\sigma_{z}^{2}}\left[\begin{array}{ccc}
A^{2} \sum_{n=0}^{N-1} n^{2} & 0 & A^{2} \sum_{n=0}^{N-1} n \\
0 & N & 0 \\
A^{2} \sum_{n=0}^{N-1} n & 0 & A^{2} N
\end{array}\right]
$$

The CRLB for the unknown parameters is obtained from the diagonal elements of the inverse of the Fisher information matrix:

$$
\operatorname{var}\left\{\hat{\alpha}_{i}\right\} \geq \frac{1}{\mathbf{J}\{\alpha\}}=\left[\begin{array}{ccc}
\frac{6}{\operatorname{snr} N\left(N^{2}-1\right)} & 0 & \frac{-3}{\operatorname{snr} N(N+1)} \\
0 & \frac{\sigma_{z}^{2}}{2 N} & 0 \\
\frac{-3}{\operatorname{snr} N(N+1)} & 0 & \frac{2 N-1}{\operatorname{snr} N(N+1)}
\end{array}\right]
$$

where snr is the signal to noise ratio given by:

$$
s n r=\frac{A^{2}}{\sigma_{z}^{2}}
$$

Therefore,

$$
\operatorname{var}(\hat{\omega}) \geq \frac{6}{\operatorname{snr} N\left(N^{2}-1\right)}
$$




$$
\operatorname{var}(\hat{A}) \geq \frac{\sigma_{z}^{2}}{2 N}
$$

and

$$
\operatorname{var}(\hat{\phi}) \geq \frac{2 N-1}{\operatorname{snr} N(N+1)}
$$




\section{Appendix B}

\section{Spectral Estimation Theory}

\section{B.1 Spectrum}

The term spectrum is used to describe the frequency-domain representation of data obtained from a invertible transformation of a time-domain signal. Gardner [31] also defines the term statistical spectrum, used to describe the spectrum created from averaging or smoothing of the data to reduce the effects of random parameters such as noise.

In digital systems, where data is analyzed in blocks of discrete-time observations, the spectrum is commonly generated via the standard periodogram [3]. The Fast Fourier Transform is typically used to generate the periodogram due to computational efficiency. However, it is biased and therefore not a consistent estimator of the true power spectral density [32]. 


\section{B.2 The Fourier Transform}

The Fourier Transform is a generalization of the Fourier Series and states that any time domain function can be represented by an infinite number of sinusoid functions[33]. The Fourier Transform $\mathcal{F}\{\}$ maps the time-domain representation of the signal $\mathrm{x}(\mathrm{t})$ to the corresponding frequency domain function $\mathrm{X}(\mathrm{f})$. In doing so, the Fourier Transform identifies the amplitudes and frequencies of the complex sinusoidal components of $\mathrm{x}(\mathrm{t})$. The Fourier Transform is given by:

$$
\mathcal{F}\{x(t)\}=X(f)=\int_{-\infty}^{\infty} x(t) e^{-j \Omega t} d t
$$

where $\mathrm{j}$ is the imaginary number, $\mathrm{f}$ is frequency in hertz and $\mathrm{t}$ is the time in seconds.

The inverse Fourier Transform $\mathcal{F}^{-1}\{\}$ maps the frequency domain representation of signal $\mathrm{X}(\mathrm{f})$ to the time domain representation $\mathrm{x}(\mathrm{t})$. The inverse Fourier Transform is given by:

$$
\mathcal{F}^{-1}\{X(f)\}=X(t)=\int_{-\infty}^{\infty} X(f) e^{j \Omega t} d f
$$

\section{B.3 The Discrete Fourier Transform}

The Fourier Transform is limited since a closed form solution is required for both the time-domain and frequency-domain expressions of the signal. In many digital receiver applications, the input signal has multiple unknown parameters.

The Discrete Fourier Transform (DFT), an approximation of the Fourier Transform, is used to generate the spectrum of digitized data. The DFT and its compliment, 
the Inverse Discrete Fourier Transform (IDFT) are given by:

$$
X[k]=\sum_{n=0}^{N-1} x[n] e^{-j \omega n}
$$

and

$$
x[n]=\frac{1}{N} \sum_{k=0}^{N-1} X[k] e^{j \omega n}
$$

where the digitized data $x[n], n=0, \ldots, N-1$, are assumed to be uniformly sampled at a rate $f_{s}$ with sampling interval $t_{s}=1 / f_{s}$. The discrete frequency components of the DFT occur at $\omega=2 \pi k / N t_{s}$, where $\mathrm{k}=0, \ldots, \mathrm{N}-1$.

The DFT of a sinusoidal signal $A \cos \left(\omega_{0} n+\theta\right)$, is a pair of impulses at frequencies $-\omega_{0}$ and $\omega_{0}$. In DFT analysis, windowing and the sample rate will have an important effect in how the signal will be represented in the frequency-domain [34].

\section{B.3.1 The Fast Fourier Transform}

The Fast Fourier Transform (FFT) is an efficient implementation of the DFT. The DFT requires $N^{2}$ operations, resulting in considerable computational burden for large DFT calculations. The FFT however, divides the N-point DFT into smaller DFTs, which are divided into smaller DFTs, etc... The end result is a reduction in computational complexity from $N^{2}$ to $N \log _{2} N$.

\section{B.3.2 Windowing and the Discrete Fourier Transform}

The input to the DFT is a block of $\mathrm{N}$ samples extracted from a larger sequence of

digital data. The block extraction is equivalent to multiplying the input data by a 
rectangular window of length $\mathrm{N}$. The windowing creates sharp discontinuities at the edges of the window, which are problematic. The FFT assumes periodic input data, with an integer number of periods within the window length. When a non-integer number of signal periods is observed within the window, discontinuities are assumed which are not present in the actual signal.

In the frequency-domain, the windowing operation is a convolution of a sinc function with the Fourier Transform of the input signal. The rectangular window function is defined:

$$
w_{\text {rect }}[n]= \begin{cases}1: & 0 \leq n<N-1 \\ 0 & : \text { otherwise }\end{cases}
$$

The rectangular window is illustrated in Figure B.1.a.

The Discrete Fourier Transform of the rectangular window function is the discrete sinc function [15], which is given by:

$$
\begin{aligned}
W_{\text {rect }}[k] & =\sum_{n=0}^{N-1} x[n] e^{-j \omega n} \\
& =\sum_{n=0}^{N-1} e^{-j \omega n} \\
& =\frac{1-e^{-j \omega N}}{1-e^{-j \omega}} \\
& =\frac{e^{-\frac{j \omega N}{2}}}{e^{-\frac{j \omega}{2}}}\left[\frac{e^{\frac{j \omega N}{2}}-e^{-\frac{j \omega N}{2}}}{e^{\frac{j \omega}{2}}-e^{-\frac{j \omega}{2}}}\right] \\
& =e^{-j \frac{\omega(N-1)}{2}} \frac{\sin \left(\frac{\omega N}{2}\right)}{\sin \left(\frac{\omega}{2}\right)}
\end{aligned}
$$

The frequency response of the rectangular window function is illustrated in Figure 

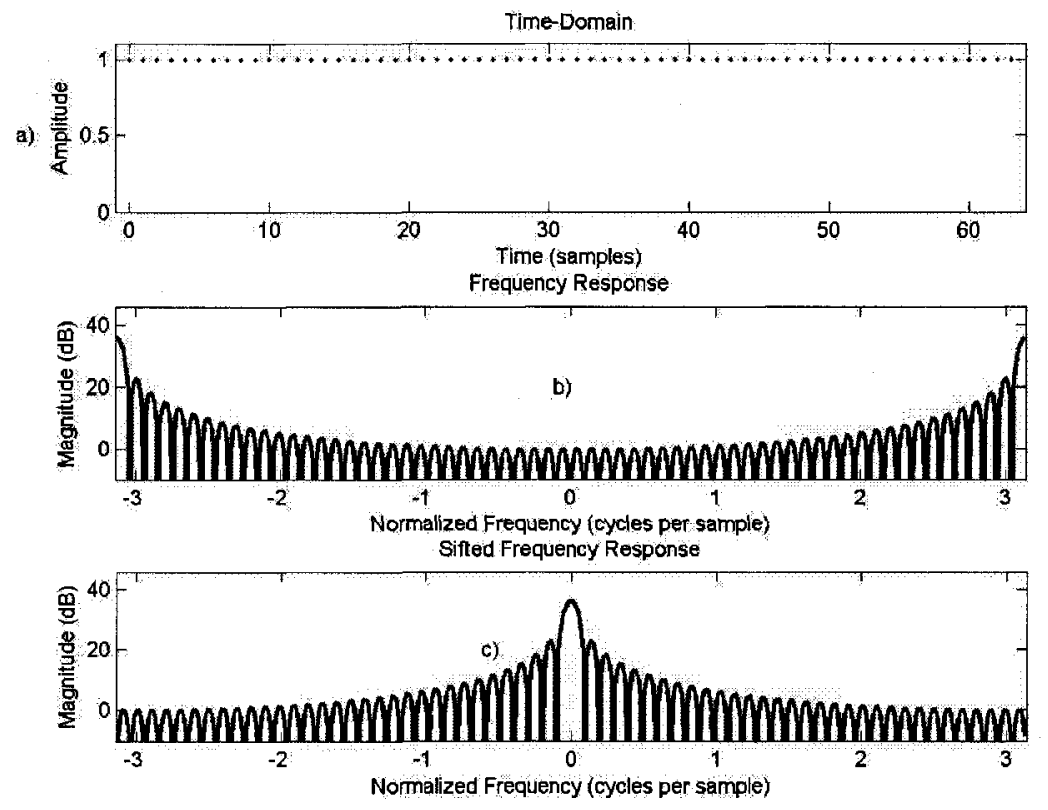

Figure B.1: The rectangular window function for $\mathrm{N}=64$. a) Time-Domain b) Frequency Response c) Shifted Frequency Response

B.1.b. The frequency response of the FFT is periodic and shifting the frequency axis provides a better illustration of the window frequency response. The shifted frequency response is presented in Figure B.1.c.

When the signal of interest is a sinusoid, the convolution with the sinc function results in a smearing of the impulses in the frequency-domain. The frequency of a rectangular windowed sinusoid is illustrated in Figure B.2.

The two main effects of windowing are reduced resolution and increased spectral leakage. The reduced spectral resolution is a function of the main lobe width of the rectangular window function. The leakage is a function of the relative amplitude of the sidelobes with respect to the window's main lobe. 

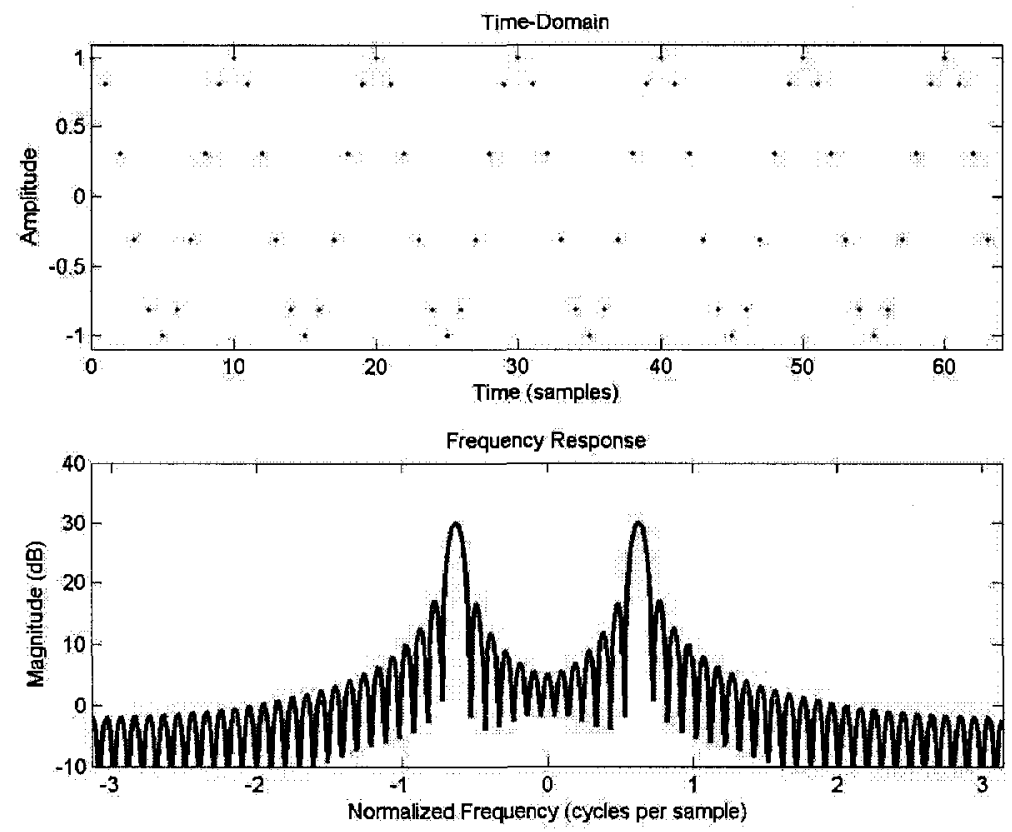

Figure B.2: Time and Frequency Response of a Rectangular Windowed Sinusoid, for $f_{0}=0.1$ and $\mathrm{N}=64$.

\section{B.3.3 Sampling and the Discrete Fourier Transform}

The input signal to a DFT is periodic, and the output spectrum is also periodic with a period $1 / N T$. Additionally, the sample rate must be greater than twice the bandwidth of the input signal to maintain the Nyquist Theorem. Failure to meet the Nyquist Theorem will result in aliasing.

Additionally, the sampling of a windowed waveform for DFT processing can produce distorted results. The source of this distortion is an uneven response to signals within a specific bin of the DFT, due to the mainlobe structure of the window function. This distortion is referred to as scalloping loss [9] and the maximum loss is observed when the input signal is located directly between two adjacent frequency bins. Figure B.3 illustrates the scalloping loss for the rectangular window. The rectangular window displays the greatest scalloping loss of traditional windows. 


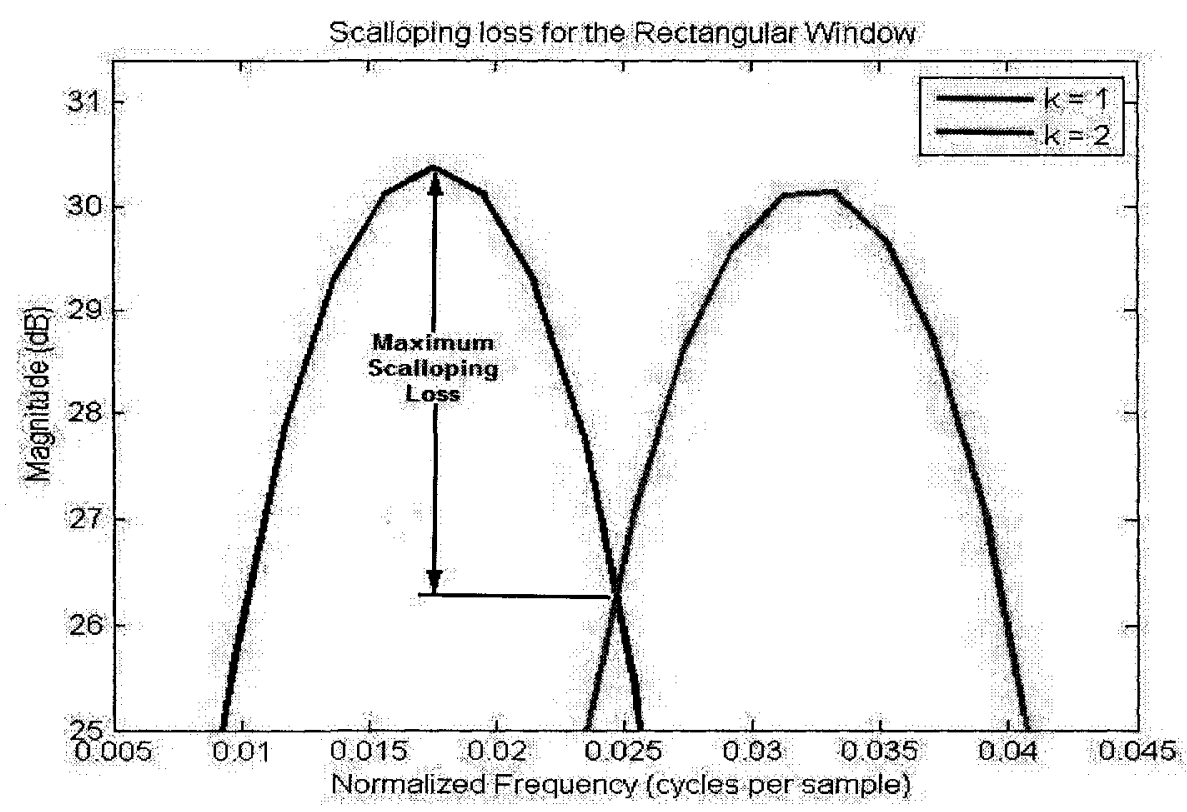

Figure B.3: Scalloping loss for the Rectangular Window, where $\mathrm{N}=64$.

\section{B.3.4 Equivalent Noise Bandwidth}

The Equivalent Noise Bandwidth (ENBW) relates the mean and variance of the noise power of an alternative windowed system to that of a rectangular windowed system. The ENBW is given by:

$$
E B W N=\frac{1}{N} \frac{\sum_{n=0}^{N-1} w[n]^{2}}{\left(\sum_{n=0}^{N-1} w[n]\right)^{2}}
$$

\section{B.4 Alternative Windows for Spectral Estimation}

The location of the impulses in the frequency bins of the DFT output is a function of the ADC sample rate. When the period of the input signal is not matched to the length of the window, distortion will occur due to discontinuities at the edges of the window. When analyzing signals with unknown parameters, it is not practical 
to match the window length to the period of the input signal. However, alternate windows can be used to minimize the effects of discontinuities at the window edges. The effects of various windows on spectral response has been studied in detail [15] [3] [31].

Table B.1: Window functions of the Rectangular, Blackmanm Hann and Hamming windows.

\begin{tabular}{|l|c|l|}
\hline Window & $\mathrm{x}[\mathrm{n}]$ & $X[k]$ \\
\hline Rectangular & 1 & $W_{\text {rect }}[k]$ \\
\hline \multirow{3}{*}{ Blackman } & $0.42-0.5 \cos \left(\frac{2 \pi n}{N}\right)+0.08 \cos \left(\frac{4 \pi n}{N}\right)$ & $\begin{array}{l}0.42 W_{\text {rect }}[k] \\
-0.25\left(W_{\text {rect }}[k-1]+W_{\text {rect }}[k+1]\right) \\
\end{array}$ \\
\hline Hann & $0.5-0.5 \cos \left(\frac{2 \pi n}{N}\right)$ & $0.04\left(W_{\text {rect }}[k-2]+W_{\text {rect }}[k+2]\right)$ \\
& & $-0.25\left(W_{\text {rect }}[k]\right.$ \\
\hline Hamming & $0.54-0.46 \cos \left(\frac{2 \pi n}{N}\right)$ & $\left.0.27 W_{\text {rect }}[k]+W_{\text {rect }}[k+1]\right)$ \\
& & $-0.23\left(W_{\text {rect }}[k-1]+W_{\text {rect }}[k+1]\right)$ \\
\hline
\end{tabular}

The performance of the rectangular and Hann windows will be further investigated in this thesis and their characteristics are present here. Table B.1 defines the timedomain and frequency response functions of the windows, Figure B.4 illustrates the window functions for $\mathrm{N}=64$ and Table B.2 presents key properties of the windows.

Table B.2: Key window parameters for the Rectangular Blackman, Hann and Hamming window Functions.

\begin{tabular}{|l|c|c|c|c|}
\hline Window & $\begin{array}{c}\text { Highest } \\
\text { Sidelobe } \\
\text { Level }(\mathrm{dB})\end{array}$ & $\begin{array}{c}\text { Asymptotic Sidelobe } \\
\text { Decay Rate } \\
\text { (dB per octave) }\end{array}$ & $\begin{array}{c}\text { Equivalent Noise } \\
\text { Bandwidth } \\
\text { (bins) }\end{array}$ & $\begin{array}{c}3 \mathrm{~dB} \\
\text { Bandwidth } \\
\text { (bins) }\end{array}$ \\
\hline Rectangular & -13 & -6 & 1.00 & 1.21 \\
\hline Blackman & -58 & -18 & 1.73 & 2.35 \\
\hline Hann & -32 & -18 & 1.50 & 2.00 \\
\hline Hamming & -43 & -6 & 1.36 & 1.81 \\
\hline
\end{tabular}

A tradeoff in sidelobe performance is made to minimize interference from closely located (in frequency) interferers and ones further out. Typically, this decision is influenced by the spectral environment of the signal. 

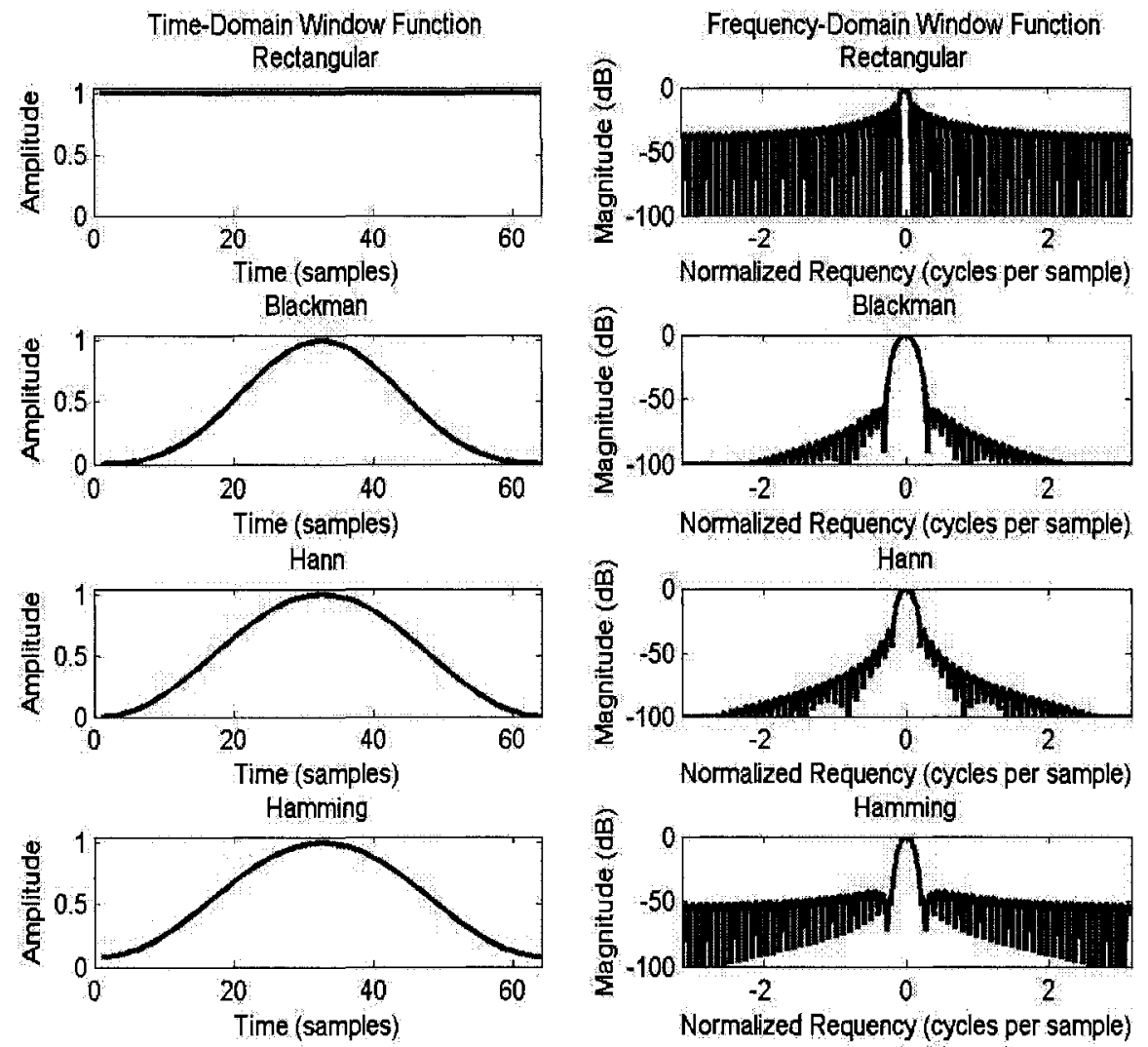

Figure B.4: Time and frequency response of the Rectangular, Blackman, Hann and Hamming window functions, for $\mathrm{N}=64$. 


\section{B.5 Zero Padding}

Zero Padding is the appending of zeros to a data sequence. Given a data sequence of

length $N$, typically LN zeros where $L=2^{i-1}$ are appended to the end of the original data sequence prior to performing the DFT operation. The resulting DFT function will be

$$
X[k]=\sum_{n=0}^{L N-1} x[n] e^{-\frac{j \omega n}{L}}=\sum_{n=0}^{N-1} x[n] e^{-\frac{j \omega n}{L}}
$$

if we substitute $k=L k^{\prime}$ : we get

$$
X\left[k^{\prime}\right]=\sum_{n=0}^{N-1} x[n] e^{-j \omega^{\prime} n}
$$

The result is a spectrum with $L N$ spectral components as opposed to the original $N$ components. Tsui [33] remarks that the amplitude and phase of the original $N$ components of the spectrum are unchanged, but the zero padding interpolates the spectrum between the original components. Although the frequency resolution is dependant on the true block length $N$, zero padding increases the accuracy of estimating the peaks of the spectral components [15].

\section{B.6 Parseval's Theorem}

An important property of the Fourier Transform is Parseval's Theorem. The theorem states that for a given signal $\mathrm{x}(\mathrm{t})$, the energy in the time domain is equal to the 
energy in the frequency domain. Parseval's Theorem is given by:

$$
\int_{-\infty}^{\infty}|x(t)|^{2} d t=\int_{-\infty}^{\infty}|X(f)|^{2} d f
$$

The corresponding discrete form of Parseval's equation is given by:

$$
\sum_{n=0}^{N-1}|x[n]|^{2}=\sum_{k=0}^{N-1} \frac{1}{N}|X[k]|^{2}
$$

\section{B.7 Power Spectral Density}

Stationary stochastic processes have infinite energy; therefore the Fourier Transform does not exist[14]. An alternative, the Fourier Transform of the autocorrelation function, provides a distribution of the signal power as a function of frequency. This spectral representation is referred to as the power spectral density and is given by:

$$
\Phi(f)=\int_{-\infty}^{\infty} R_{x_{1} x_{1}} e^{-j \Omega t} d t
$$

\section{B.8 The Periodogram}

The periodogram is an estimate of the true PSD. The periodogram can be generated by taking the DFT of the autocorrelation series or by computing it directly with a the DFT output series of the input process. The direct form of the periodogram, also referred to as the sample spectrum, is given by:

$$
P[k]=\frac{1}{N}\left|\sum_{n=0}^{N-1} x[n] e^{-j \omega n}\right|^{2}=\frac{1}{N}|X[k]|^{2}
$$




\section{B.8.1 Modified Periodogram}

When an alternative to the rectangular window function is used to compute the periodogram, the spectral operation is referred to as a modified periodogram [34]. The output of the modified periodogram is given by:

$$
P[k]=\frac{\frac{1}{N}\left|\sum_{n=0}^{N-1} w[n] x[n] e^{-j \omega n}\right|^{2}}{\frac{1}{N}\left|\sum_{n=0}^{N-1} w[n]\right|^{2}}
$$

\section{B.8.2 Averaged Periodograms}

The bias and variance of the periodogram are important properties for accurate spectral estimation. The input data for the DFT are windowed with a rectangular function. Therefore, the mean of the periodogram is the convolution of the true PSD with a Bartlett (triangular) window:

$$
w[n]=\left\{\begin{aligned}
\frac{n}{N} & : \quad 0 \leq n \leq N \\
2-\frac{n}{N} & : \quad N<n<2 N-1 \\
0 & : \text { otherwise }
\end{aligned}\right.
$$

For finite $\mathrm{N}$, the windowing of the periodogram distorts the output, resulting in a biased estimator. However, as $\mathrm{N} \rightarrow \infty$, the periodogram approaches the true PSD and therefore is asymptotically unbiased.

The variance of the periodogram is not a function of $N$ [15] and does not converge to zero as $\mathrm{N} \rightarrow \infty$. Increasing $\mathrm{N}$ does increase the resolution of the periodogram; however, the variance is not reduced and $\mathrm{P}[\mathrm{k}]$ is therefore not a consistent estimator of the PSD.

Reduced variance is achieved through periodogram averaging. The input data 
is divided into $M$ smaller sequences of length $\mathrm{L}$. The periodogram is calculated for each sequence of data and the periodogram bins are averaged. The individual periodograms are assumed to be independent and identically distributed random variables. Therefore, the mean of the average periodogram is equal to the mean of the original periodogram. The variance of $\mathrm{M}$ averaged independent and identically distributed random variables is $1 / \mathrm{M}$ the variance of the the individual random variables [35]. Therefore, the variance of the periodogram is reduced by a factor $M$ when $M$ periodograms are averaged.

In general, the reduced variance comes at a cost of lower spectral resolution and increased bias (due to the smaller periodogram size). The trade-off between higher spectral resolution and lower estimator variance will be greatly influenced by the signal environment. The Bartlett and Welch methods of spectral averaging will be further investigated in this thesis.

\section{The Bartlett Method}

The Bartlett method [30],[15] separates a data sequence of length $\mathrm{N}$ into $\mathrm{M}$ continuous, non-overlapping sequences of length L. The periodogram is calculated for each sequence and the $M$ resulting periodograms are averaged to produce the Bartlett averaged periodogram. The Bartlett averaged periodogram is given by:

$$
P_{B}[k]=\frac{1}{M} \sum_{m=0}^{M-1} P_{m}[k], \quad k=0, \ldots, L-1
$$

where $P_{m}[k]$ is the periodogram of a data segment given by: 


$$
P_{m}\left[k^{\prime}\right]=\sum_{n=m L}^{(m+1) L-1} x[n] e^{-j \omega^{\prime} n}, \quad m=0, \ldots, M-1
$$

The Bartlett method is depicted in Figure B.5

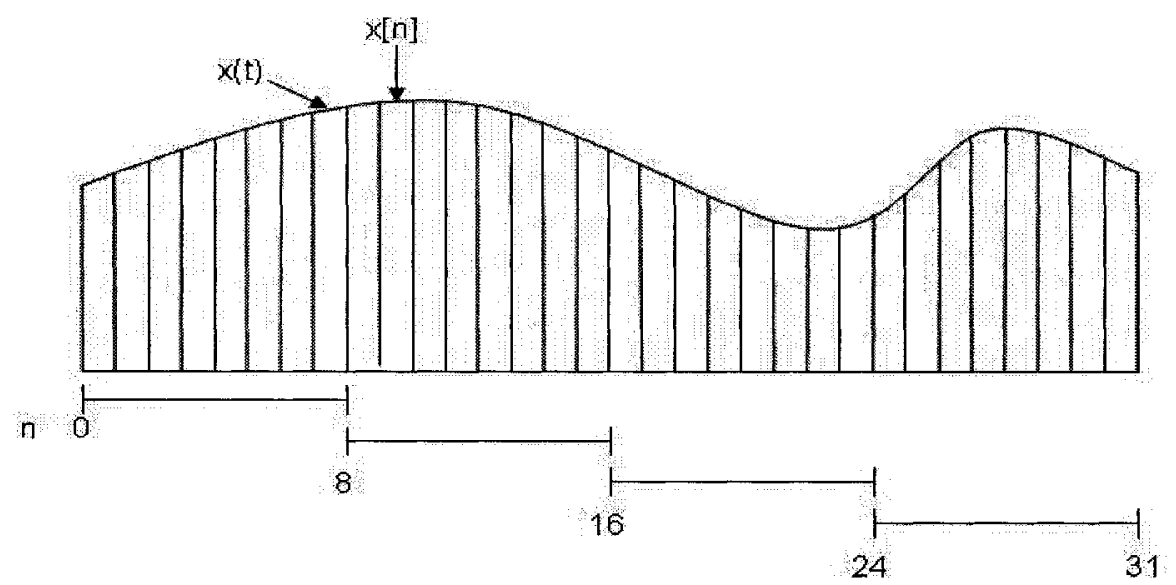

Figure B.5: The Bartlett periodogram averaging method for $\mathrm{N}=32, \mathrm{~L}=8$ and $\mathrm{M}$ $=4$.

Using the Bartlett method, the variance of the periodogram is reduce by a factor $\mathrm{M}$ and the spectral resolution is reduced by a factor $\mathrm{M}$. The trade-off between estimator variance and spectral resolution for the Bartlett method for a noisy sinusoid with $\mathrm{SNR}=-10 \mathrm{~dB}$ is depicted in Figure B.6.

\section{The Welch Method}

Welch improves on the Bartlett method by allowing the data sequences to overlap and by applying window functions on the data prior to calculating the periodogram of the data segments. The overlapping increases the number of periodograms averaged, resulting in decreased variance for the periodogram. The windowing decreases spectral leakage and estimator bias, at the cost of decreased frequency resolution. 
a)
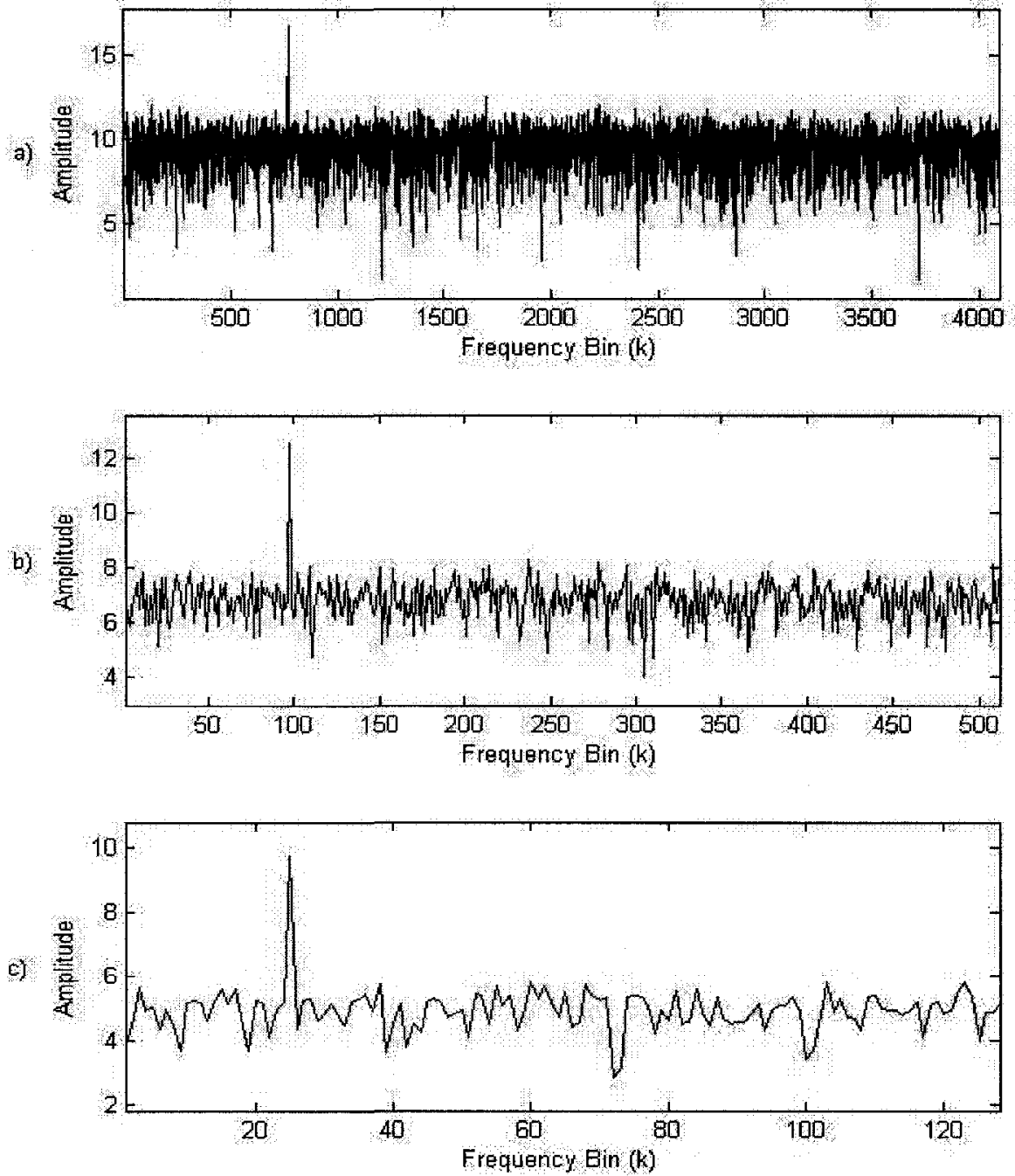

Figure B.6: a) The periodogram for $\mathrm{N}=4096$. b) The Bartlett averaged periodogram for $\mathrm{M}=8$ and $\mathrm{L}=512$. c) Bartlett averaged periodogram for $\mathrm{M}=32$ and $\mathrm{L}=128$. 
The Welch averaged periodogram is given by:

$$
P_{W}[k]=\frac{1}{M} \sum_{m=0}^{M-1} P_{m}[k] \quad k=0, \ldots, L-1
$$

where $P_{m}[k]$ is the periodogram of a data segment given by:

$$
P_{m}\left[k^{\prime}\right]=\sum_{n=\frac{m L}{2}}^{\frac{(m+2) L}{2}-1} w[n] x[n] e^{-j \omega^{\prime} n}, \quad m=0, \ldots, M-1
$$

and $\mathrm{w}[\mathrm{n}]$ is the Hann window defined in Table B.1. The Welch averaged periodogram method is illustrated in Figure B.7

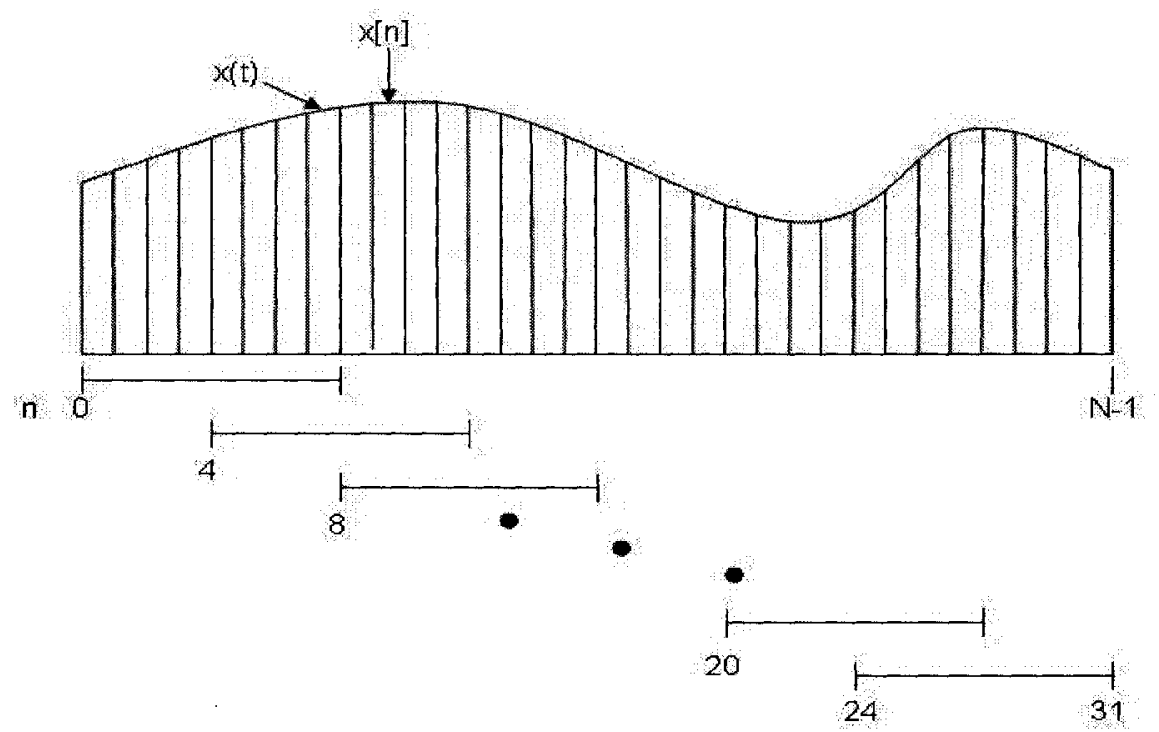

Figure B.7: The Welch periodogram averaging method for $\mathrm{N}=32, \mathrm{~L}=8, \mathrm{M}=7$ and overlap $=50 \%$.

Welch specifically proposed the use of the Hann window with $50 \%$ overlap[15]. The $50 \%$ overlap strategy is a highly efficient implementation of the Welch averaged periodogram, making it a desirable technique for reducing estimator variance. With 
$50 \%$ overlap, the data segments can be extracted directly from the original data sequence. Marple [15] has shown that the minimum variance, when using the Hann window, is obtained with an overlap of $65 \%$. However, the variance of the $50 \%$ overlap method is only $8 \%$ higher than the $62 \%$ overlap case and is a simpler implementation. The overlapping of the Welch averaged periodogram method results in a greater number of periodogram averages (N/L for Bartlett method as opposed to 2N/L 1 for the Welch Method). As with the Bartlett method, the variance of the Welch averaged periodogram will be reduced by approximately $1 / \mathrm{M}$. The Welch averaged periodogram for the same scenario used in the Bartlett discussion is presented in Figure B.8. As expected, the variance of the Welch method is lower than that of the Bartlett method for the same data sequence. 
a)
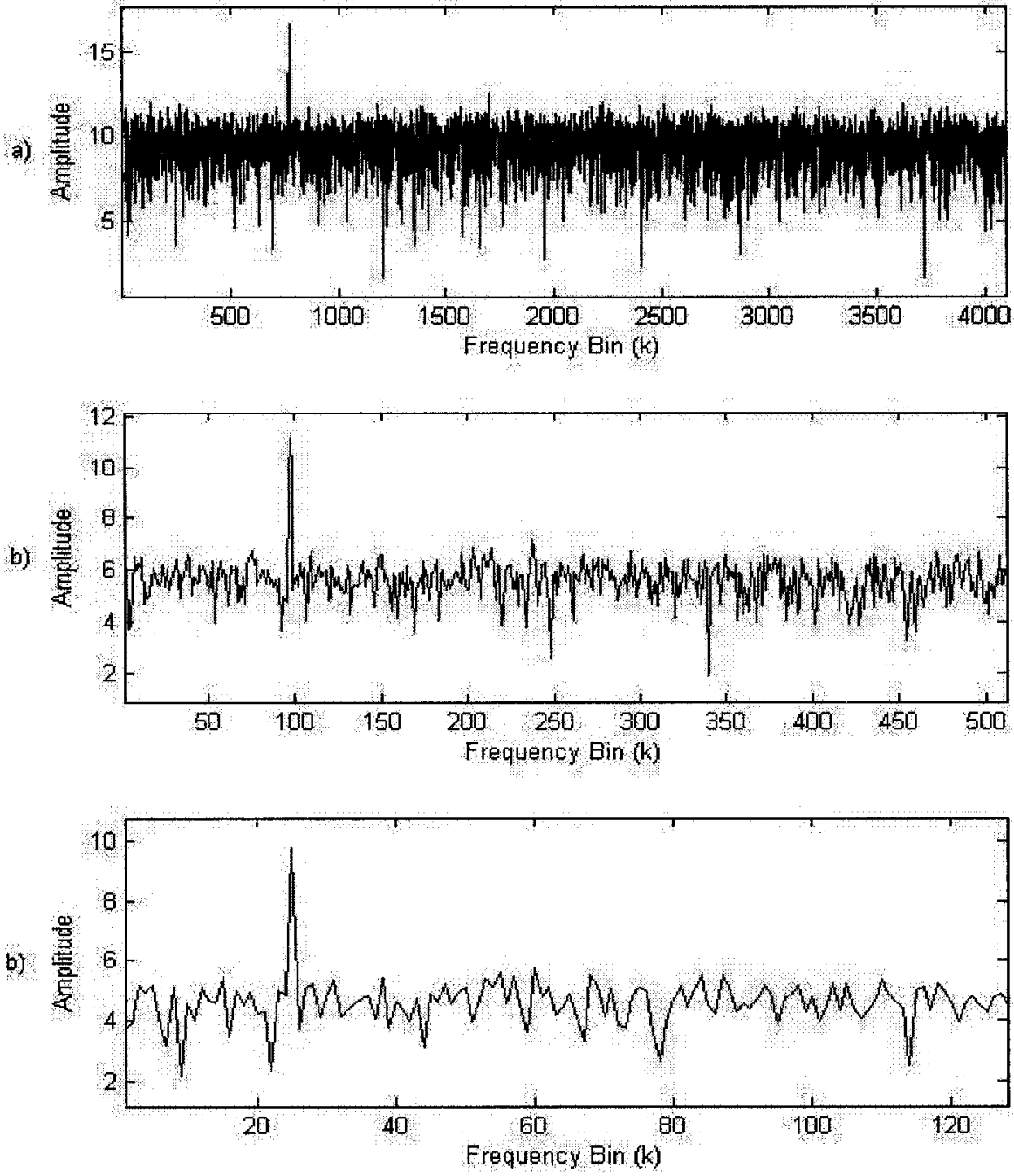

Figure B.8: a) The periodogram for $\mathrm{N}=4096$. b) The Welch averaged periodogram for $\mathrm{M}=15$ and $\mathrm{L}=512$. c) The Welch averaged periodogram for $\mathrm{M}=63$ and $\mathrm{L}=$ 128. 


\section{Appendix C}

\section{Characteristic polynomial for a $4 \times 4$}

\section{Matrix}

The following is a calculation of the characteristic polynomial, for the case of a $4 \times 4$ matrix A.

The characteristic polynomial is calculated by solving the characteristic equation, given by:

$$
\operatorname{det}(\mathbf{A}-\mathbf{I} \lambda)=0
$$

Let the $4 \times 4$ square matrix $\mathbf{A}$ be:

$$
\mathbf{A}=\left[\begin{array}{cccc}
a_{11} & a_{12} & a_{13} & a_{14} \\
a_{21} & a_{22} & a_{23} & a_{24} \\
a_{31} & a_{32} & a_{33} & a_{34} \\
a_{41} & a_{42} & a_{43} & a_{44}
\end{array}\right]
$$


then equation C.1 becomes:

$$
\begin{aligned}
\operatorname{det}(A-I \lambda) & =\left|\begin{array}{cccc}
a_{11}-\lambda & a_{12} & a_{13} & a_{14} \\
a_{21} & a_{22}-\lambda & a_{23} & a_{24} \\
a_{31} & a_{32} & a_{33}-\lambda & a_{34} \\
a_{41} & a_{42} & a_{43} & a_{44}-\lambda
\end{array}\right| \\
& =B-C+D-E
\end{aligned}
$$

The components $\mathbf{B}, \mathbf{C}, \mathbf{D}$ and $\mathbf{E}$ are given by:

$$
\begin{aligned}
B= & \left(a_{11}-\lambda\right)\left|\begin{array}{ccc}
a_{22}-\lambda & a_{23} & a_{24} \\
a_{32} & a_{33}-\lambda & a_{34} \\
a_{42} & a_{43} & a_{44}-\lambda
\end{array}\right| \\
= & \lambda^{4}-\left[a_{11}+a_{22}+a_{33}+a_{44}\right] \lambda^{3} \\
& +\left[a_{11} a_{22}+a_{11} a_{33}+a_{11} a_{44}+a_{22} a_{33}+a_{22} a_{44}+a_{33} a_{44}-a_{23} a_{32}\right. \\
& \left.-a_{24} a_{42}-a_{34} a_{43}\right] \lambda^{2} \\
& +\left[a_{11} a_{23} a_{32}+a_{11} a_{24} a_{42}+a_{11} a_{34} a_{43}+a_{22} a_{34} a_{43}+a_{24} a_{33} a_{42}+a_{23} a_{32} a_{44}\right. \\
& \left.-a_{11} a_{22} a_{33}-a_{11} a_{22} a_{44}-a_{11} a_{33} a_{44}-a_{22} a_{33} a_{44}-a_{23} a_{34} a_{42}-a_{24} a_{43} a_{32}\right] \lambda \\
& +a_{11} a_{22} a_{33} a_{44}+a_{11} a_{23} a_{34} a_{42}+a_{11} a_{24} a_{43} a_{32}-a_{11} a_{22} a_{34} a_{43} \\
& -a_{11} a_{23} a_{32} a_{44}-a_{11} a_{24} a_{33} a_{42}
\end{aligned}
$$




$$
\begin{aligned}
C= & a_{12}\left|\begin{array}{ccc}
a_{21} & a_{23} & a_{24} \\
a_{31} & a_{33}-\lambda & a_{34} \\
a_{41} & a_{43} & a_{44}-\lambda
\end{array}\right| \\
= & a_{12} a_{21} \lambda^{2} \\
& +\left[a_{12} a_{23} a_{31}+a_{12} a_{24} a_{41}-a_{12} a_{21} a_{33}-a_{12} a_{21} a_{44}\right] \lambda \\
& +a_{12} a_{21} a_{33} a_{44}+a_{12} a_{23} a_{34} a_{41}+a_{12} a_{24} a_{43} a_{31}-a_{12} a_{21} a_{34} a_{43} \\
& -a_{12} a_{23} a_{31} a_{44}-a_{12} a_{24} a_{33} a_{41}
\end{aligned}
$$

$$
\begin{aligned}
D= & a_{13}\left|\begin{array}{ccc}
a_{21} & a_{22}-\lambda & a_{24} \\
a_{31} & a_{32} & a_{34} \\
a_{41} & a_{42} & a_{44}-\lambda
\end{array}\right| \\
= & -a_{13} a_{31} \lambda^{2} \\
& +\left[a_{13} a_{31} a_{22}+a_{13} a_{31} a_{44}-a_{13} a_{32} a_{21}-a_{13} a_{34} a_{41}\right] \lambda \\
& +a_{13} a_{32} a_{21} a_{44}+a_{13} a_{34} a_{41} a_{22}+a_{13} a_{31} a_{24} a_{42}-a_{13} a_{34} a_{42} a_{21} \\
& -a_{13} a_{31} a_{22} a_{44}-a_{13} a_{32} a_{24} a_{41}
\end{aligned}
$$

$$
\begin{aligned}
E= & a_{14}\left|\begin{array}{ccc}
a_{21} & a_{22}-\lambda & a_{23} \\
a_{31} & a_{32} & a_{33}-\lambda \\
a_{41} & a_{42} & a_{43}
\end{array}\right| \\
= & a_{14} a_{41} \lambda^{2} \\
& +\left[a_{14} a_{42} a_{21}+a_{14} a_{43} a_{31}-a_{14} a_{41} a_{22}-a_{14} a_{41} a_{33}\right] \lambda \\
& +a_{14} a_{43} a_{32} a_{21}+a_{14} a_{41} a_{22} a_{33}+a_{14} a_{42} a_{23} a_{31}-a_{14} a_{42} a_{21} a_{33} \\
& -a_{14} a_{43} a_{31} a_{22}-a_{14} a_{41} a_{23} a_{32}
\end{aligned}
$$


Therefore, inserting equations C.5 to C.8 into equation C.3, the characteristic polynomial is given by:

$$
\begin{aligned}
\operatorname{det}(A-I \lambda) & =\lambda^{4}-\left[a_{11}+a_{22}+a_{33}+a_{44}\right] \lambda^{3} \\
& +\left[a_{11} a_{22}+a_{11} a_{33}+a_{11} a_{44}+a_{22} a_{33}+a_{22} a_{44}+a_{33} a_{44}\right. \\
& \left.-a_{23} a_{32}-a_{24} a_{42}-a_{34} a_{43}-a_{12} a_{21}-a_{13} a_{31}-a_{14} a_{41}\right] \lambda^{2} \\
& +\left[a_{11} a_{23} a_{32}+a_{11} a_{24} a_{42}+a_{11} a_{34} a_{43}+a_{22} a_{34} a_{43}+a_{24} a_{33} a_{42}\right. \\
& +a_{23} a_{32} a_{44}-a_{11} a_{22} a_{33}-a_{11} a_{22} a_{44}-a_{11} a_{33} a_{44}-a_{22} a_{33} a_{44} \\
& -a_{23} a_{34} a_{42}-a_{24} a_{43} a_{32}-a_{12} a_{23} a_{31}-a_{12} a_{24} a_{41}+a_{12} a_{21} a_{33} \\
& +a_{12} a_{21} a_{44}+a_{13} a_{31} a_{22}+a_{13} a_{31} a_{44}-a_{13} a_{32} a_{21}-a_{13} a_{34} a_{41} \\
& \left.-a_{14} a_{42} a_{21}-a_{14} a_{43} a_{31}+a_{14} a_{41} a_{22}+a_{14} a_{41} a_{33}\right] \lambda \\
& +a_{11} a_{22} a_{33} a_{44}+a_{11} a_{23} a_{34} a_{42}+a_{11} a_{24} a_{43} a_{32}-a_{11} a_{22} a_{34} a_{43} \\
& -a_{11} a_{23} a_{32} a_{44}-a_{11} a_{24} a_{33} a_{42}-a_{12} a_{21} a_{33} a_{44}-a_{12} a_{23} a_{34} a_{41} \\
& -a_{12} a_{24} a_{43} a_{31}+a_{12} a_{21} a_{34} a_{43}+a_{12} a_{23} a_{31} a_{44}+a_{12} a_{24} a_{33} a_{41} \\
& +a_{13} a_{32} a_{21} a_{44}+a_{13} a_{34} a_{41} a_{22}+a_{13} a_{31} a_{24} a_{42}-a_{13} a_{34} a_{42} a_{21} \\
& -a_{13} a_{31} a_{22} a_{44}-a_{13} a_{32} a_{24} a_{41}-a_{14} a_{43} a_{32} a_{21}-a_{14} a_{41} a_{22} a_{33} \\
& -a_{14} a_{42} a_{23} a_{31}+a_{14} a_{42} a_{21} a_{33}+a_{14} a_{43} a_{31} a_{22}+a_{14} a_{41} a_{23} a_{32}
\end{aligned}
$$




$$
\begin{aligned}
& =\lambda^{4}-(\operatorname{trace} A) \lambda^{3}+\left[\sum_{i=1}^{4} \sum_{j>i}^{4} a_{i i} a_{j j}-\sum_{i=1}^{4} \sum_{j>i}^{4} a_{i j} a_{j i}\right] \lambda^{2} \\
& -\left[\sum_{i=1}^{4} \sum_{j>i}^{4} \sum_{k>j}^{4} a_{i i} a_{j j} a_{k k}-\sum_{i=1}^{4} \sum_{\substack{j>i \\
j \neq i}}^{4} \sum_{\substack{k>j \\
k \neq i}}^{4} a_{i i} a_{j k} a_{k j}\right. \\
& \left.-\sum_{i=1}^{4} \sum_{j>i}^{4} \sum_{\substack{k>i \\
k \neq j}}^{4} a_{i j} a_{j k} a_{k i}\right] \lambda \\
& +\prod_{i=1}^{4} a_{i i}-\sum_{i=1}^{4} \sum_{j>i}^{4} \sum_{\substack{k=1 \\
k \neq i, j}}^{4} \sum_{\substack{l>k \\
l \neq i, j, k}}^{4} a_{i i} a_{j j} a_{k l} a_{l k}+\sum_{i=1}^{4} \sum_{\substack{j=1 \\
j \neq i}}^{4} \sum_{\substack{k>j \\
k \neq i, j}}^{4} \sum_{\substack{l>k \\
l \neq i, j, k}}^{4} a_{i i} a_{j k} a_{k l} a_{l j} \\
& -\sum_{i=2}^{4} \sum_{\substack{j>2 \\
j \neq i}}^{4} \sum_{\substack{k=2 \\
k \neq i, j}}^{4} a_{1 i} a_{i j} a_{j k} a_{k 1}+\sum_{i=2}^{4} \sum_{\substack{j=2 \\
j \neq i}}^{4} \sum_{\substack{k>j \\
k \neq 1, i, j}}^{4} a_{1 i} a_{i 1} a_{j k} a_{k j} \\
& =\lambda^{4}-(\operatorname{trace} A) \lambda^{3}+\left[\sum_{i=1}^{4} \sum_{j>i}^{4} a_{i i} a_{j j}-\sum_{i=1}^{4} \sum_{j>i}^{4} a_{i j} a_{j i}\right] \lambda^{2} \\
& -\left[\sum_{i=1}^{4} \sum_{j>i}^{4} \sum_{k>j}^{4} a_{i i} a_{j j} a_{k k}-\sum_{i=1}^{4} \sum_{\substack{j>i \\
j \neq i}}^{4} \sum_{\substack{k>j \\
k \neq i}}^{4} a_{i i} a_{j k} a_{k j}\right. \\
& \left.-\sum_{i=1}^{4} \sum_{j>i}^{4} \sum_{\substack{k>i \\
k \neq j}}^{4} a_{i j} a_{j k} a_{k i}\right] \lambda+\operatorname{det}(\mathbf{A})
\end{aligned}
$$




\section{Appendix D}

\section{Solving Quartic Equations}

In performing the eigen-decomposition of a multichannel PSD matrix, a poynomial equation must be solved. For large polynomial equations, iterative techniques are typically used to provide an approximate solution. For the case of a four channel PSD matrix, a quartic equation must be solved and a closed form solution does exists. The procedure described in this appendix details the closed form solution for a solving a quartic equation.

The quartic equation is given by:

$$
a_{0} x^{4}+b_{0} x^{3}+c_{0} x^{2}+d_{0} x+e_{0}=0
$$




\section{D.1 Convert the quartic equation to a depressed quartic equation}

The first step in solving the quartic equation is to convert equation D.1 to a depressed quartic equation of the form:

$$
x^{4}++\alpha x^{2}+\beta x+\gamma=0
$$

First, the quartic equation is simplified by dividing both sides of equation D.1 by $a_{0}$ producing:

$$
\begin{aligned}
\frac{a_{0}}{a_{0}} x^{4}+\frac{b_{0}}{a_{0}} x^{3}+\frac{c_{0}}{a_{0}} x^{2}+\frac{d_{0}}{a_{0}} x+\frac{e_{0}}{a_{0}} & =0 \\
x^{4}+b x^{3}+c x^{2}+d x+e & =0
\end{aligned}
$$

Next, the $x^{3}$ term is removed by introducing a variable substitution of $\mathrm{x}=\mathrm{y}-\frac{b}{4}$ producing:

$$
\left(y-\frac{b}{4}\right)^{4}+b\left(y-\frac{b}{4}\right)^{3}+c\left(y-\frac{b}{4}\right)^{2}+d\left(y-\frac{b}{4}\right)+e=0
$$

Expanding the powers and collecting the terms yields the depressed quartic equation:

$$
\begin{aligned}
y^{4}+\left(\frac{-3 b^{2}}{8}+c\right) y^{2}+\left(\frac{b^{3}}{8}-\frac{b c}{2}+d\right) y & + \\
\left(\frac{-3 b^{4}}{256}+\frac{b^{2} c}{16}-\frac{b d}{4}+e\right) & =0 \\
y^{4}+\alpha y^{2}+\beta y+\gamma & =0
\end{aligned}
$$

where the new coefficients in terms of the initial coefficients are: 
- $\alpha=\frac{-3 b_{0}^{2}}{8 a_{0}^{2}}+\frac{c_{0}}{a_{0}}$

- $\beta=\frac{b_{0}^{3}}{8 a_{0}^{3}}-\frac{b_{0} c_{0}}{2 a_{0}^{2}}+\frac{d_{0}}{a_{0}}$

- $\gamma=\frac{-3 b_{0}^{4}}{256 a_{0}^{4}}+\frac{b_{0}^{2} c_{0}}{16 a_{0}^{3}}-\frac{b_{0} d_{0}}{4 a_{0}^{2}}+\frac{e_{0}}{a_{0}}$

When the constant $\beta=0$, it is observed that equation D.6 simplifies to a quadratic equation in $y^{2}$. For this special case, the four roots are given by:

$$
x_{1,2,3,4}=\frac{ \pm \sqrt{-\alpha \pm \sqrt{\alpha^{2}-4 \gamma}}}{2}-\frac{b}{4}
$$

\section{D.2 Factor the quartic equations into two quadratic equations}

The strategy for solving the quartic equation requires the quartic equation be factored into two quadratic equations as follows:

$$
\begin{aligned}
y^{4}+\alpha y^{2}+\beta y+\gamma & =\left(y^{2}+p y+q\right)\left(y^{2}+r y+s\right) \\
& =y^{4}+(p+r) y^{3}+(q+s+p r) y^{2}+(p s+q r) y+q s
\end{aligned}
$$

by equating the coefficients, we get:

- $0=\mathrm{p}+\mathrm{r}$

- $\alpha=\mathrm{q}+\mathrm{s}+\mathrm{pr}$

- $\beta=\mathrm{ps}+\mathrm{qr}$

- $\gamma=\mathrm{qs}$ 


\section{D.3 Create an expression for the quadratic coeffi- cients}

It is observed that $\mathrm{p}=-\mathrm{r}$. Substituted this result into the remaining coefficient equations produces the following relationships:

- $\alpha+p^{2}=\mathrm{q}+\mathrm{s}$

- $\frac{\beta}{p}=\mathrm{s}-\mathrm{q}$

- $\gamma=\mathrm{qs}$

Next, q and s are eliminated as follows':

$$
\begin{aligned}
\left(\alpha+p^{2}\right)^{2}-\left(\frac{\beta}{p}\right)^{2} & =(s+q)^{2}-(s-q)^{2} \\
& =4 s q \\
\left(\alpha+p^{2}\right)^{2}-\left(\frac{\beta}{p}\right)^{2} & =4 \gamma
\end{aligned}
$$

Expanding the terms in equation D.9 produces the following relationship:

$$
\begin{aligned}
\alpha^{2}+2 \alpha p^{2}+p^{4}-\frac{\beta^{2}}{p^{2}} & =4 \gamma \\
\alpha^{2} p^{2}+2 \alpha p^{4}+p^{6}-\beta^{2} & =4 \gamma p^{2} \\
p^{6}+2 \alpha p^{4}+\left(\alpha^{2}-4 \gamma\right) p^{2}-\beta^{2} & =0
\end{aligned}
$$

By substituting $\mathrm{P}=p^{2}$ into equation D.10, the resulting cubic equation is produced:

$$
P^{3}+2 \alpha P^{2}+\left(\alpha^{2}-4 \gamma\right) P-\beta^{2}=0
$$


A change of variables is performed to consolidate and simplify equation D.11 producing:

$$
P^{3}+a a P^{2}+b b P+c c=0
$$

where,

- $\mathrm{aa}=2 \alpha$

- $\mathrm{bb}=\alpha^{2}-4 \gamma$

- $\mathrm{cc}=-\beta^{2}$

\section{D.4 Solving the cubic equation}

As with the quartic equation, the cubic equation must be converted to a depressed cubic equation of the form:

$$
X^{3}+A X+B=0
$$

Substituting $\mathrm{P}=\mathrm{X}-\frac{a a}{3}$ into equation D.12 produces the required depressed cubic equation:

$$
\begin{aligned}
\left(X-\frac{a a}{3}\right)^{3}+2 a a\left(X-\frac{a a}{3}\right)^{2}+b b\left(X-\frac{a a}{3}\right)+c c & =0 \\
X^{3}+\left(b b-\frac{a a^{2}}{3}\right) X+\left(c c+\frac{2 a a^{3}-9 a a b b}{27}\right) & =0 \\
X^{3}+A X+B & =0
\end{aligned}
$$

where,

- $\mathrm{A}=b b-\frac{a a^{2}}{3}$

- $\mathrm{B}=c c+\frac{2 a a^{3}-9 a a b b}{27}$ 
To solve the cubic, two new variables are constructed based on A and B as follows:

$$
\begin{aligned}
& Q=\frac{A}{3} \\
& R=\frac{B}{2}
\end{aligned}
$$

When $Q^{3}-R^{2} \geq 0$, the cubic equation has three real roots, otherwise it has one real root and two complex roots [23]. The method for computing the roots is different for each condition, as presented in the following subsections.

\section{D.4.1 Solving a cubic equation with three real roots}

When the cubic has three real roots, the following trigonometric identity is used [36]:

$$
4 \cos ^{3}(\theta)-3 \cos (\theta)-\cos (3 \theta)=0
$$

Let

$$
X=m \cos (\theta)
$$

then equation D.13 becomes:

$$
\begin{aligned}
X^{3}+A X+B & =m^{3} \cos ^{3}(\theta)+A m \cos (\theta)+B=0 \\
& =4 \cos ^{3}(\theta)-3 \cos (\theta)-\cos (3 \theta)
\end{aligned}
$$

Therefore,

$$
\frac{4}{m^{3}}=\frac{-3}{A m}=\frac{-\cos (3 \theta)}{B}
$$


From equation D.21, the following relationships are obtained:

$$
m=2 \sqrt{\frac{-A}{3}}=-2 \sqrt{Q}
$$

and

$$
\begin{aligned}
\cos (3 \theta)=\frac{3 B}{A m} & =\frac{3 B}{A 2} \sqrt{\frac{3}{-A}}=\frac{R}{\sqrt{Q^{3}}} \\
3 \theta & =\cos ^{-1}\left(\frac{R}{\sqrt{Q^{3}}}\right)
\end{aligned}
$$

Substituting equation D.22 and equation D.23 into equation D.18, the principle root of the depressed cubic equation is given by:

$$
X_{1}=-2 \sqrt{Q} \cos \left(\frac{\theta}{3}\right)
$$

Therefore, the principle root of the original cubic equation is given by:

$$
P_{1}=-2 \sqrt{Q} \cos \left(\frac{\theta}{3}\right)-\frac{a a}{3}
$$

and the remaining roots are given by:

$$
\begin{aligned}
& P_{2}=-2 \sqrt{Q} \cos \left(\frac{\theta+2 \pi}{3}\right)-\frac{a a}{3} \\
& P_{3}=-2 \sqrt{Q} \cos \left(\frac{\theta+4 \pi}{3}\right)-\frac{a a}{3}
\end{aligned}
$$




\section{D.4.2 Solving a cubic equation with one real root and two complex roots}

When the cubic has one real root and two complex roots, it is solved by generating a quadratic equation based on the terms of the cubic and solving its roots. First, the depressed cubic equation is factored:

$$
X^{3}+A X+B=(v-u)^{3}+3 u v(v-u)+\left(u^{3}-v^{3}\right)=0
$$

where,

- $\mathrm{X}=\mathrm{v}-\mathrm{u}$

- $\mathrm{A}=3 \mathrm{uv}$

- $\mathrm{B}=u^{3}-v^{3}$

Substituting $\mathrm{A}=3$ uv into $\mathrm{B}=u^{3}-v^{3}$ produces:

$$
\begin{aligned}
B & =u^{3}-\left(\frac{A}{3 u}\right)^{3} \\
27 u^{3} B & =27 u^{6}-A^{3} \\
0 & =27 u^{6}-27 B u^{3}-A^{3}
\end{aligned}
$$

The resulting equation is a quadratic equation which can be solved easily. The roots of the quadratic equation are given by:

$$
\begin{aligned}
u^{3} & =\frac{B}{2} \pm \sqrt{\frac{B^{2}}{4}+\frac{A^{3}}{27}} \\
u & =\sqrt[3]{\frac{B}{2} \pm \sqrt{\frac{B^{2}}{4}+\frac{A^{3}}{27}}}
\end{aligned}
$$


From equation D.30 it is observed that there are six possible solutions for $u$. Care must be taken in computing $u$. If $A=0$, then the plus sign of the plus/minus option in equation D.30 must be selected to avoid a division by zero in equation D.29 (By default one can always select the positive sign).

Once a solution for $\mathrm{u}$ has been obtained, the value for $\mathrm{P}$ is computed. Manipulating the expressions $\mathrm{P}=\mathrm{X}-\frac{a a}{3}, \mathrm{X}=\mathrm{v}-\mathrm{u}$ and $\mathrm{A}=3 \mathrm{uv}$, the solution for the principle root $P_{1}$ is given by:

$$
P_{1}=\frac{A}{3 u}-u-\frac{a a}{3}
$$

The second and third roots are transformations of the principle root:

$$
\begin{aligned}
& P_{2}=P_{1}\left(\frac{-1}{2}+\frac{\sqrt{3} i}{2}\right) \\
& P_{3}=P_{1}\left(\frac{-1}{2}+\frac{\sqrt{3} i}{2}\right)
\end{aligned}
$$

\section{D.5 Solving the quartic equation}

The principle root $\left(\mathrm{P}_{1}\right)$ is used to solve the quartic equation as follows:

$$
P=p^{2}
$$

Next, the values for $\mathrm{r}, \mathrm{s}$ and $\mathrm{q}$ are computed using:

$$
\begin{aligned}
r & =-p \\
s & =\frac{\alpha+p^{2}+\frac{\beta}{p}}{2} \\
q & =\frac{\alpha+p^{2}-\frac{\beta}{p}}{2}
\end{aligned}
$$


We now have all the coefficients for equation D.8:

$$
y^{4}+\alpha y^{2}+\beta y+\gamma=\left(y^{2}+p y+q\right)\left(y^{2}+r y+s\right)
$$

The roots of the quartic equation are easily calculated by solving the two quadratic equations giving:

$$
\begin{aligned}
& \operatorname{root}_{1}=\frac{-p+\sqrt{p^{2}-4 q}}{2} \\
& \operatorname{root}_{2}=\frac{-p-\sqrt{p^{2}-4 q}}{2} \\
& \operatorname{root}_{3}=\frac{-r+\sqrt{r^{2}-4 s}}{2} \\
& \operatorname{root}_{4}=\frac{-r-\sqrt{r^{2}-4 s}}{2}
\end{aligned}
$$

